\begin{tabular}{|c|l|}
\hline Title & On classical solutions of the compressible Navier-Stokes equations with nonnegative initial densities \\
\hline Author(s) & Cho, Yonggeun; Kim, Hyunseok \\
\hline Citation & $\begin{array}{l}\text { manuscripta mathematica, 120(1), 91-129 } \\
\text { https://doi.org/L0.1007/300229-006-0637-y }\end{array}$ \\
\hline Issue Date & 2006-05 \\
\hline Doc URL & http://hdl.handle.net/2115/14421 \\
\hline Rights & The original publication is available at www.springerlink.com \\
\hline Type & article (author version) \\
\hline File Information & ManuscriptaMath_v120p91.pdf \\
\hline
\end{tabular}

Instructions for use 


\title{
ON CLASSICAL SOLUTIONS OF THE COMPRESSIBLE NAVIER-STOKES EQUATIONS WITH NONNEGATIVE INITIAL DENSITIES
}

\author{
YONGGEUN CHO AND HYUNSEOK KIM
}

\begin{abstract}
We study the Navier-Stokes equations for compressible barotropic fluids in a bounded or unbounded domain $\Omega$ of $\mathbf{R}^{3}$. We first prove the local existence of solutions $(\rho, u)$ in $C\left(\left[0, T_{*}\right] ;\left(\rho^{\infty}+H^{3}(\Omega)\right) \times\left(D_{0}^{1} \cap D^{3}\right)(\Omega)\right)$ under the assumption that the data satisfies a natural compatibility condition. Then deriving the smoothing effect of the velocity $u$ in $t>0$, we conclude that $(\rho, u)$ is a classical solution in $\left(0, T_{* *}\right) \times \Omega$ for some $T_{* *} \in\left(0, T_{*}\right]$. For these results, the initial density needs not be bounded below away from zero and may vanish in an open subset (vacuum) of $\Omega$.
\end{abstract}

\section{IntRoduction}

The motion of a viscous compressible barotropic fluid in a domain $\Omega$ of $\mathbf{R}^{3}$ can be described by the Naiver-Stokes equations

$$
\begin{array}{cc}
\rho_{t}+\operatorname{div}(\rho u)=0 & \text { in } \quad(0, T) \times \Omega, \\
(\rho u)_{t}+\operatorname{div}(\rho u \otimes u)+L u+\nabla p=\rho f & \text { in } \quad(0, T) \times \Omega, \\
L u=-\mu \Delta u-(\lambda+\mu) \nabla \operatorname{div} u, \quad p=p(\rho) &
\end{array}
$$

and the initial and boundary conditions

$$
\begin{gathered}
\left.(\rho, u)\right|_{t=0}=\left(\rho_{0}, u_{0}\right) \quad \text { in } \quad \Omega, \quad u=0 \quad \text { on } \quad(0, T) \times \partial \Omega, \\
\rho(t, x) \rightarrow \rho^{\infty}, \quad u(t, x) \rightarrow 0 \quad \text { as } \quad|x| \rightarrow \infty, \quad(t, x) \in(0, T) \times \Omega .
\end{gathered}
$$

Here we denote by $\rho, p$ and $u$ the unknown density, pressure and velocity fields of the fluid, respectively. $f$ denotes a given external force and the constants $\mu, \lambda$ are the viscosity coefficients. We assume that the pressure $p=p(\rho)$ is a smooth function of the density $\rho$ and the viscosity coefficients $\mu$ and $\lambda$ satisfy the natural physical restrictions $\mu>0$ and $3 \lambda+2 \mu \geq 0$ so that $L=-\mu \Delta-(\lambda+\mu) \nabla$ div is a strongly elliptic operator. Moreover, $(0, T) \times \Omega$ is the time-space domain for the evolution of the fluid, where $T$ is a finite positive number and $\Omega$ is either a bounded domain in $\mathbf{R}^{3}$ with smooth boundary or a usual unbounded domain such as the whole space $\mathbf{R}^{3}$, the half space $\mathbf{R}^{2} \times \mathbf{R}_{+}$and an exterior domain with smooth boundary. Of course, if $\Omega$ is a bounded domain (or the whole space), then the condition (1.5) at

2000 Mathematics Subject Classification. 35Q30, 76N10.

Key words and phrases. classical solution, compressible Navier-Stokes equations, vacuum.

The authors were supported by Japan Society for the Promotion of Science under JSPS Postdoctoral Fellowship For Foreign Researchers. 
infinity (or the boundary condition in (1.4) respectively) is unnecessary and should be neglected.

In this paper, we study the initial boundary value problem (simply IBVP) (1.1)(1.5) with nonnegative initial densities.

Under the crucial assumption that the initial density $\rho_{0}$ is bounded below away from zero, the first existence results for the IBVP (1.1)-(1.5) were obtained by Nash [20], Itaya [13] and Tani [24]. They applied a fixed point argument or the method of successive approximations in Hölder spaces to prove the local (in time) existence of classical solutions even for more general heat-conducting fluid models. Then using delicate energy methods in Sobolev spaces, Matsumura and Nishida showed in their pioneering papers $[18,19]$ that the classical solutions exist globally in time provided that the data are small in some sense. See also the papers $[6,12,23,27,28,29]$ for some further local or global results in case of positive densities.

On the other hand, the existence of weak or strong solutions has been proved in rather recent works even for the general case of nonnegative initial densities. In fundamental works $[16,17]$, Lions developed an existence theory of global (in time) weak solutions to the IBVP (1.1)-(1.5). Then Lions' theory has been improved by several authors to deduce more general results; see [7, 8, 9, 10, 14, 15] for details. The very recent papers $[2,3,4]$ by Choe and the authors are devoted to establishing some local existence results on strong solutions. Among other things, we showed in $[2,3]$ (see also the paper [21] by Salvi and Straškraba) that if the initial data $\rho_{0}$, $u_{0}$ satisfy the regularity condition

$$
\rho_{0}-\rho^{\infty} \in H^{2}, \quad \rho^{\infty} \in \overline{\mathbf{R}}_{+}, \quad \rho_{0} \geq 0 \quad \text { in } \quad \Omega, \quad u_{0} \in D_{0}^{1} \cap D^{2}
$$

and the compatibility condition

$$
L u_{0}+\nabla p\left(\rho_{0}\right)=\rho_{0}^{\frac{1}{2}} g_{1} \quad \text { in } \quad \Omega \text { for some } g_{1} \in L^{2}
$$

then there exist a small time $T_{*} \in(0, T)$ and a unique strong solution $(\rho, u)$ to the IBVP (1.1)-(1.5) such that

$$
\begin{gathered}
\rho-\rho^{\infty} \in C\left(\left[0, T_{*}\right] ; H^{2}\right), \quad u \in C\left(\left[0, T_{*}\right] ; D_{0}^{1} \cap D^{2}\right) \cap L^{2}\left(0, T_{*} ; D^{3}\right), \\
\rho_{t} \in C\left(\left[0, T_{*}\right] ; H^{1}\right), \quad u_{t} \in L^{2}\left(0, T_{*} ; D_{0}^{1}\right) \quad \text { and } \sqrt{\rho} u_{t} \in L^{\infty}\left(0, T_{*} ; L^{2}\right) .
\end{gathered}
$$

Throughout this paper, we adopt the following simplified notations for the standard homogeneous and inhomogeneous Sobolev spaces.

$$
\begin{aligned}
& L^{r}=L^{r}(\Omega), \quad D^{k, r}=\left\{v \in L_{l o c}^{1}(\Omega):|v|_{D^{k, r}}<\infty\right\} \\
& W^{k, r}=L^{r} \cap D^{k, r}, H^{k}=W^{k, 2}, \quad D^{k}=D^{k, 2} \\
& D_{0}^{1}=\left\{v \in L^{6}(\Omega):|v|_{D_{0}^{1}}<\infty \text { and } v=0 \text { on } \partial \Omega\right\} \\
& H_{0}^{1}=L^{2} \cap D_{0}^{1}, \quad|v|_{D^{k, r}}=\left|\nabla^{k} v\right|_{L^{r}} \quad \text { and } \quad|v|_{D_{0}^{1}}=|\nabla v|_{L^{2}} .
\end{aligned}
$$

Then it follows from the classical Sobolev embedding results that

$$
|v|_{L^{6}} \leq C|v|_{D_{0}^{1}}, \quad|v|_{L^{\infty}} \leq C|v|_{W^{1,4}} \quad \text { and } \quad|v|_{L^{\infty}} \leq C|v|_{D_{0}^{1} \cap D^{2}}
$$


Hereafter we use the obvious notation

$$
|\cdot|_{X \cap Y}=|\cdot|_{X}+|\cdot|_{Y} \quad \text { for (semi-)normed spaces } \quad X, Y
$$

and $C$ denotes a generic positive constant depending only on the fixed constants $\mu, \lambda, T$ and the norms of $p=p(\cdot)$ and $f$. We also denote by $H^{-1}$ the dual space of $H_{0}^{1}$ with $\left\langle\cdot, \cdot>\right.$ being the dual paring of $H^{-1}$ and $H_{0}^{1}$. A detailed study of homogeneous Sobolev spaces may be found in Galdi's book [11].

The main purpose of this paper is to prove the local existence of classical solutions to the IBVP (1.1)-(1.5) with nonnegative initial densities. First we prove the existence of solutions in $C\left(\left[0, T_{*}\right] ;\left(\rho^{\infty}+H^{3}\right) \times\left(D_{0}^{1} \cap D^{3}\right)\right)$ under a stronger compatibility condition than (1.7) on the data.

Theorem 1.1. Assume that

$$
\begin{gathered}
\rho_{0}-\rho^{\infty} \in H^{3}, \quad \rho^{\infty} \in \overline{\mathbf{R}}_{+}, \quad \rho_{0} \geq 0 \quad \text { in } \quad \Omega, \quad u_{0} \in D_{0}^{1} \cap D^{3}, \\
f \in L^{2}\left(0, T ; H^{2}\right), \quad f_{t} \in L^{2}\left(0, T ; L^{2}\right) \quad \text { and } \quad p=p(\cdot) \in C^{3}\left(\overline{\mathbf{R}}_{+}\right) .
\end{gathered}
$$

Assume further that the data $\rho_{0}, u_{0}, f$ satisfy the compatibility condition

$$
L u_{0}+\nabla p\left(\rho_{0}\right)=\rho_{0}\left(f(0)+g_{2}\right) \text { for some } g_{2} \in D_{0}^{1} \text { with } \sqrt{\rho}_{0} g_{2} \in L^{2} \text {. }
$$

Then there exist a small time $T_{*} \in(0, T)$ and a unique strong solution $(\rho, u)$ to the IBVP (1.1)-(1.5) such that

$$
\begin{gathered}
\rho-\rho^{\infty} \in C\left(\left[0, T_{*}\right] ; H^{3}\right), \quad u \in C\left(\left[0, T_{*}\right] ; D_{0}^{1} \cap D^{3}\right) \cap L^{2}\left(0, T_{*} ; D^{4}\right), \\
u_{t} \in L^{\infty}\left(0, T_{*} ; D_{0}^{1}\right) \cap L^{2}\left(0, T_{*} ; D^{2}\right) \quad \text { and } \quad \sqrt{\rho} u_{t} \in L^{\infty}\left(0, T_{*} ; L^{2}\right) .
\end{gathered}
$$

Remark 1.2. From the continuity equation (1.1), it follows immediately that

$$
\rho_{t} \in C\left(\left[0, T_{*}\right] ; H^{2}\right) \quad \text { and } \quad \rho_{t t} \in L^{\infty}\left(0, T_{*} ; L^{2}\right) \cap L^{2}\left(0, T_{*} ; H^{1}\right) .
$$

Note that the hypotheses of Theorem 1.1 imply (1.6) and (1.7) with $g_{1}=$ $\sqrt{\rho}_{0}\left(f(0)+g_{2}\right) \in L^{2}$. Hence the existence of a unique local solution $(\rho, u)$ with the regularity (1.8) was already proved in $[2,3]$ and our new theorem shows that $(\rho, u)$ has some additional regularity if the data satisfy a stronger compatibility condition (1.10). It is easy to show that (1.10) is also necessary for the existence of solutions with the regularity (1.11). In fact, let $(\rho, u)$ be a solution to the $\operatorname{IBVP}(1.1)-(1.5)$ with the regularity (1.11). Then since $u_{t} \in L^{\infty}\left(0, T_{*} ; D_{0}^{1}\right)$ and $\sqrt{\rho} u_{t} \in L^{\infty}\left(0, T_{*} ; L^{2}\right)$, there is a sequence $\left\{t_{k}\right\}, t_{k} \rightarrow 0$, such that $u_{t}\left(t_{k}\right) \rightarrow g$ in $D_{0}^{1}$ for some $g \in D_{0}^{1}$ with $\sqrt{\rho}(0) g \in L^{2}$. Hence letting $t=t_{k} \rightarrow 0$ in the momentum equation (1.2), we readily obtain

$$
L u(0)+\nabla p(\rho(0))=\rho(0)(f(0)-u(0) \cdot \nabla u(0)-g),
$$

which implies then that

$$
L u(0)+\nabla p(\rho(0))=\rho(0)\left(f(0)+g_{2}\right)
$$


where $g_{2}=-u(0) \cdot \nabla u(0)-g$. Noting that $\rho(0)=\rho_{0}, u(0)=u_{0}, g_{2} \in D_{0}^{1}$ and $\sqrt{\rho}(0) g_{2} \in L^{2}$, we conclude that the compatibility condition (1.10) is necessary for the existence of solutions with the regularity (1.11).

In case that $\rho_{0}$ has a positive lower bound and $u_{0}$ has the additional integrability condition $u_{0} \in L^{2}$, Theorem 1.1 can be proved applying the method of successive approximations or a fixed point argument as in [1, 13, 18, 24, 29]. Our proof of the theorem is based on the method of successive approximations, whose general strategy may be described as follows. First we consider a linearized problem for the IBVP (1.1)-(1.5) and solve it successively to construct a sequence of approximate solutions. Then we derive some uniform bounds for approximate solutions and finally prove the convergence of the sequence to a solution to the original nonlinear problem. A detailed proof of Theorem 1.1 following this strategy is provided in Section 4 .

Next, we prove the existence of classical solutions to the IBVP (1.1)-(1.5). Let $(\rho, u)$ be a solution to (1.1)-(1.5) satisfying the regularity in Theorem 1.1. Then in view of the Sobolev embedding results, we have

$$
(\rho, u) \in C\left(\left[0, T_{*}\right] ; C^{1}(\bar{\Omega})\right) \quad \text { and } \quad \rho_{t} \in C\left(\left[0, T_{*}\right] \times \bar{\Omega}\right),
$$

which implies that $(\rho, u)$ satisfies (1.1), (1.3), (1.4) and (1.5) in a classical sense. But in order to conclude that (1.2) is satisfied in a classical sense, we need to prove further regularity of $u$. In case that $\rho_{0}$ is bounded below away from zero, that is, $\delta=\inf _{\Omega} \rho_{0}>0$, it follows from (1.12) that $\rho \geq \frac{1}{2} \delta>0$ on $\left[0, T_{* *}\right] \times \bar{\Omega}$ for some $T_{* *} \in\left(0, T_{*}\right]$ and the momentum equation (1.2) can be rewritten as

$$
u_{t}+\rho^{-1} L u=f-u \cdot \nabla u-\rho^{-1} \nabla p(\rho)
$$

in $\left(0, T_{* *}\right) \times \Omega$. Hence by virtue of the smoothing effect of solutions of parabolic equations, we deduce that $\left(\nabla^{2} u, u_{t}\right) \in C\left(\left(0, T_{* *}\right] \times \bar{\Omega}\right)$ and $(\rho, u)$ is a classical solution of $(1.2)$ in $\left(0, T_{* *}\right) \times \Omega$. For details, see Lemma 2.4 in the next section and the paper [18] by Matsumura and Nishida. However the smoothing effect of the velocity $u$ in $t>0$ is not obvious for the general case of nonnegative initial densities because (1.2) is no more parabolic in the region where the density vanishes.

Nevertheless, using the same method as in the proof of Theorem 1.1, we can prove the following result.

Theorem 1.3. In addition to (1.9) and (1.10), we assume that

$$
\begin{gathered}
t^{\frac{1}{2}} f \in L^{\infty}\left(0, T ; H^{2}\right), \quad t^{\frac{1}{2}} f_{t} \in L^{\infty}\left(0, T ; L^{2}\right), \quad t^{\frac{1}{2}} f_{t t} \in L^{2}\left(0, T ; H^{-1}\right) \\
t f_{t} \in L^{\infty}\left(0, T ; H^{1}\right), \quad t f_{t t} \in L^{2}\left(0, T ; L^{2}\right) \\
t^{\frac{3}{2}} f_{t t} \in L^{\infty}\left(0, T ; L^{2}\right) \quad \text { and } \quad t^{\frac{3}{2}} f_{t t t} \in L^{2}\left(0, T ; H^{-1}\right)
\end{gathered}
$$


Then there exist a small time $T_{*} \in(0, T)$ and a unique strong solution $(\rho, u)$ to the IBVP (1.1)-(1.5) such that

$$
\begin{gathered}
\rho-\rho^{\infty} \in C\left(\left[0, T_{*}\right] ; H^{3}\right), \quad u \in C\left(\left[0, T_{*}\right] ; D_{0}^{1} \cap D^{3}\right) \cap L^{2}\left(0, T_{*} ; D^{4}\right), \\
u_{t} \in L^{\infty}\left(0, T_{*} ; D_{0}^{1}\right) \cap L^{2}\left(0, T_{*} ; D^{2}\right), \quad \sqrt{\rho} u_{t t} \in L^{2}\left(0, T_{*} ; L^{2}\right) ; \\
t^{\frac{1}{2}} u \in L^{\infty}\left(0, T_{*} ; D^{4}\right), \quad t^{\frac{1}{2}} u_{t} \in L^{\infty}\left(0, T_{*} ; D^{2}\right), \quad t^{\frac{1}{2}} u_{t t} \in L^{2}\left(0, T_{*} ; D_{0}^{1}\right), \\
t^{\frac{1}{2}} \sqrt{\rho} u_{t t} \in L^{\infty}\left(0, T_{*} ; L^{2}\right) ; \quad t u_{t} \in L^{\infty}\left(0, T_{*} ; D^{3}\right), \\
t u_{t t} \in L^{\infty}\left(0, T_{*} ; D_{0}^{1}\right) \cap L^{2}\left(0, T_{*} ; D^{2}\right), \quad t \sqrt{\rho} u_{t t t} \in L^{2}\left(0, T_{*} ; L^{2}\right) ; \\
t^{\frac{3}{2}} u_{t t} \in L^{\infty}\left(0, T_{*} ; D^{2}\right), \quad t^{\frac{3}{2}} u_{t t t} \in L^{2}\left(0, T_{*} ; D_{0}^{1}\right), \quad t^{\frac{3}{2}} \sqrt{\rho} u_{t t t} \in L^{\infty}\left(0, T_{*} ; L^{2}\right) .
\end{gathered}
$$

Let $(\rho, u)$ be a solution of the compressible Navier-Stokes equations (1.1)-(1.3) with the regularity (1.14). Then it is easy to show that $(\rho, u)$ is indeed a classical solution of (1.1)-(1.3) in $\left(0, T_{*}\right] \times \Omega$. First, using the standard embedding results

$$
L^{2}\left(0, T_{*} ; H^{1}\right) \cap W^{1,2}\left(0, T_{*} ; H^{-1}\right) \hookrightarrow C\left(\left[0, T_{*}\right] ; L^{2}\right)
$$

and

$$
L^{\infty}\left(0, T_{*} ; H^{1}\right) \cap W^{1,2}\left(0, T_{*} ; H^{-1}\right) \hookrightarrow C\left(\left[0, T_{*}\right] ; L^{q}\right)
$$

for any $2 \leq q<6$, we deduce from (1.13) and (1.14) that

$$
t^{\frac{1}{2}} f \in C\left(\left[0, T_{*}\right] ; W^{1,4}\right) \quad \text { and } \quad t u_{t} \in C\left(\left[0, T_{*}\right] ; D_{0}^{1} \cap D^{2}\right) .
$$

On the other hand, by virtue of the continuity equation (1.1), we can rewrite the momentum equation (1.2) as

$$
\rho u_{t}+\rho u \cdot \nabla u+L u+\nabla p(\rho)=\rho f \quad \text { in } \quad\left(0, T_{*}\right) \times \Omega,
$$

which implies that for each $t \in\left(0, T_{*}\right], u=u(t) \in D_{0}^{1} \cap D^{3}$ is a solution of the elliptic system

$$
L u=\rho\left(f-u_{t}-u \cdot \nabla u\right)-\nabla p(\rho) \equiv F \quad \text { in } \quad \Omega .
$$

Note that $t F \in C\left(\left[0, T_{*}\right] ; W^{1,4}\right)$. Hence it follows from the elliptic regularity result in [3] that

$$
t \nabla^{2} u \in C\left(\left[0, T_{*}\right] ; W^{1,4}\right) .
$$

Therefore, in view of the Sobolev embedding results, we conclude that

$$
\left(u_{t}, \nabla^{2} u\right) \in C\left(\left(0, T_{*}\right] \times \bar{\Omega}\right)
$$

and so $(\rho, u)$ is a classical solution of (1.1)-(1.3) in $\left(0, T_{*}\right] \times \Omega$.

We have considered the Navier-Stokes equations (1.1)-(1.3) for general barotropic compressible fluids including isentropic fluids as an important special class. An isentropic viscous compressible fluid is governed by the Navier-Stokes equations (1.1)-(1.3) with the density-pressure law $p=p(\cdot)$ given by

$$
p=A \rho^{\gamma} \quad \text { for some constants } \quad A>0, \quad \gamma>1 .
$$

Note that (1.15) defines a $C^{3}$-function on $\overline{\mathbf{R}}_{+}$if and only if $\gamma=2$ or $\gamma \geq 3$. Hence Theorem 1.1 and Theorem 1.3 can be used to deduce the corresponding existence results for the isentropic equations (1.1)-(1.3) and (1.15) only in case when $\gamma=2$ 
or $\gamma \geq 3$. But in some physical situations, the case $1<\gamma<2$ is most important: for instance, $\gamma=\frac{5}{3}$ in case of monatomic gases like Helium and Neon. The final goal of the paper is to prove the existence of classical solutions of the isentropic compressible Navier-Stokes equations (1.1)-(1.3) and (1.15) for general $\gamma>1$.

Theorem 1.4. Assume that the data $\rho_{0}, u_{0}, f$ satisfy the regularity condition

$$
\begin{aligned}
& \left(\rho_{0}-\rho^{\infty}, p_{0}-p^{\infty}\right) \in H^{3}, \quad \rho^{\infty} \in \overline{\mathbf{R}}_{+}, \quad \rho_{0} \geq 0 \quad \text { in } \quad \Omega, \\
& u_{0} \in D_{0}^{1} \cap D^{3}, \quad f \in L^{2}\left(0, T ; H^{2}\right) \quad \text { and } \quad f_{t} \in L^{2}\left(0, T ; L^{2}\right)
\end{aligned}
$$

and the compatibility condition

$$
L u_{0}+\nabla p_{0}=\rho_{0}\left(f(0)+g_{2}\right) \text { for some } g_{2} \in D_{0}^{1} \text { with } \sqrt{\rho}_{0} g_{2} \in L^{2},
$$

where

$$
p_{0}=A \rho_{0}^{\gamma} \quad \text { and } \quad p^{\infty}=A\left(\rho^{\infty}\right)^{\gamma} .
$$

Then there exist a small time $T_{*} \in(0, T)$ and a unique strong solution $(\rho, p, u)$ to the IBVP (1.1)-(1.5) and (1.15) such that

$$
\begin{gathered}
\left(\rho-\rho^{\infty}, p-p^{\infty}\right) \in C\left(\left[0, T_{*}\right] ; H^{3}\right), \quad u \in C\left(\left[0, T_{*}\right] ; D_{0}^{1} \cap D^{3}\right) \cap L^{2}\left(0, T_{*} ; D^{4}\right), \\
u_{t} \in L^{\infty}\left(0, T_{*} ; D_{0}^{1}\right) \cap L^{2}\left(0, T_{*} ; D^{2}\right) \quad \text { and } \sqrt{\rho} u_{t} \in L^{\infty}\left(0, T_{*} ; L^{2}\right) .
\end{gathered}
$$

Moreover, if the external force $f$ satisfies the additional regularity (1.13), then the velocity $u$ satisfies (1.14) with $T_{*}$ replaced by some $T_{* *} \in\left(0, T_{*}\right]$ and so $(\rho, p, u)$ is a classical solution of (1.1)-(1.3) and (1.15) in $\left(0, T_{* *}\right) \times \Omega$.

If $\gamma=2$ or $\gamma \geq 3$, then Theorem 1.4 is just a reformulation of Theorem 1.1 and Theorem 1.3 because

$$
\rho-\rho^{\infty} \in C\left(\left[0, T_{*}\right] ; H^{3}\right) \text { implies that } p-p^{\infty} \in C\left(\left[0, T_{*}\right] ; H^{3}\right) .
$$

But (1.16) fails to hold for general $\gamma>1$ and in fact, one major difficulty in proving Theorem 1.4 is to show that $p-p^{\infty} \in C\left(\left[0, T_{*}\right] ; H^{3}\right)$. Our proof relies heavily on the observation that since $\rho$ satisfies (1.1) and (1.4), the pressure $p=A \rho^{\gamma}$ is a solution to the linear hyperbolic problem

$$
p_{t}+u \cdot \nabla p+\gamma p \operatorname{div} u=0 \quad \text { in } \quad(0, T) \times \Omega \quad \text { and }\left.\quad p\right|_{t=0}=p_{0} \quad \text { in } \Omega,
$$

provided that $u$ is regarded as a known vector field. Hence assuming that $u$ is sufficiently regular, we can deduce from a standard regularity theory of hyperbolic equations that if $p_{0}-p^{\infty} \in H^{3}$, then $p-p^{\infty} \in C\left(\left[0, T_{*}\right] ; H^{3}\right)$. A detailed proof of Theorem 1.4 is given in the final section.

The main results in this paper are Theorem 1.3 and Theorem 1.4 which are both local existence results on classical solutions. It is then a fundamental question to ask whether the solutions exist globally in time. A negative answer was obtained by Xin [30] for the case that the spatial domain $\Omega$ is the whole space $\mathbf{R}^{3}$. He showed that there is no global classical solution to the Cauchy problem for the isentropic compressible Navier-Stokes equations with compactly supported initial density and velocity. On the other hand, Choe and the second author [5] obtained 
a global existence result on radially symmetric strong solutions of the isentropic compressible Navier-Stokes equations in bounded and unbounded annular domains. Hence it is very likely that the methods in this paper and [5] can be combined to prove the global existence of radially symmetric classical solutions with nonnegative densities. This issue will be studied in a separated paper.

The rest of this paper is organized as follows. Section 2 is devoted to a study of a linearized problem. We provide some existence and regularity results for a linear transport equation and a linear parabolic system. In Section 3, we derive some a priori estimates for solutions to the linearized problem. Applying the method of successive approximations based on these estimates, we prove Theorem 1.1 in Section 4. Finally, the proofs of Theorem 1.3 and Theorem 1.4 are given in Section 5 and Section 6 , respectively.

\section{EXISTENCE AND REGULARITY ON SOLUTIONS OF LINEAR EQUATIONS}

In this section, we obtain some existence and regularity results on solutions of a linear transport equation and a linear parabolic system, which are necessary to prove all the main theorems in the paper.

2.1. A linear transport equation. First, we consider the following linear hyperbolic problem

$$
\rho_{t}+v \cdot \nabla \rho+\rho \operatorname{div} v=0 \quad \text { in } \quad(0, T) \times \Omega \quad \text { and } \quad \rho(0)=\rho_{0} \quad \text { in } \quad \Omega,
$$

where $v$ is a known vector field in $(0, T) \times \Omega$ such that

$$
v \in C\left([0, T] ; D_{0}^{1} \cap D^{m}\right) \cap L^{2}\left(0, T ; D^{m+1}\right) \quad \text { for some integer } \quad m \geq 2 .
$$

Following the arguments in [2], we prove

Lemma 2.1. Assume that $\rho_{0}-\rho^{\infty} \in H^{m}, \rho^{\infty} \in \overline{\mathbf{R}}_{+}$and $\rho_{0} \geq 0$ in $\Omega$. Then

(i) there exists a unique solution $\rho$ to the problem (2.1) such that

$$
\rho-\rho^{\infty} \in C\left([0, T] ; H^{m}\right) \quad \text { and } \quad \rho_{t} \in C\left([0, T] ; H^{m-1}\right),
$$

(ii) the solution $\rho$ satisfies the following estimate

$$
\left|\rho(t)-\rho^{\infty}\right|_{H^{m}} \leq\left(\left|\rho_{0}-\rho^{\infty}\right|_{H^{m}}+\rho^{\infty}\right) \exp \left(C \int_{0}^{t}|v(s)|_{D_{0}^{1} \cap D^{m+1}} d s\right)
$$

for $0 \leq t \leq T$ and finally,

(iii) the solution $\rho$ is represented by the formula

$$
\rho(t, x)=\rho_{0}(U(0, t, x)) \exp \left[-\int_{0}^{t} \operatorname{div} v(s, U(s, t, x)) d s\right],
$$

where $U \in C([0, T] \times[0, T] \times \bar{\Omega})$ is the solution to the initial value problem

$$
\left\{\begin{array}{rlrl}
\frac{\partial}{\partial t} U(t, s, x) & =v(t, U(t, s, x)), & & 0 \leq t \leq T \\
U(s, s, x) & =x, \quad 0 \leq s \leq T, & x \in \bar{\Omega}
\end{array}\right.
$$


Proof. To begin with, we construct sequences $\left\{\rho_{0}^{k}\right\}$ and $\left\{v^{k}\right\}$ of more regular scalar and vector fields such that

$$
\begin{gathered}
\rho_{0}^{k}-\rho^{\infty} \in H^{m} \cap C^{m+1}(\bar{\Omega}), \\
v^{k} \in L^{2}\left(0, T ; D_{0}^{1} \cap D^{m+1}\right) \cap C^{m+1}([0, T] \times \bar{\Omega}), \\
\left|\rho_{0}^{k}-\rho_{0}\right|_{H^{m}}+\left|v^{k}-v\right|_{L^{2}\left(0, T ; D_{0}^{1} \cap D^{m+1}\right)} \rightarrow 0 \quad \text { as } \quad k \rightarrow \infty .
\end{gathered}
$$

For this purpose, we first recall that $H^{m+3}$ and $L^{2}\left(0, T ; H^{m+2}\right)$ are dense in $H^{m}$ and $L^{2}\left(0, T ; H^{m}\right)$, respectively. Then since $\rho_{0}-\rho^{\infty} \in H^{m}$ and $g=\nabla v \in L^{2}\left(0, T ; H^{m}\right)$, there exist sequences $\left\{\rho_{0}^{k}\right\}$ in $\rho^{\infty}+H^{m+3}$ and $\left\{g^{k}\right\}$ in $L^{2}\left(0, T ; H^{m+2}\right)$ such that $\rho_{0}^{k}-\rho^{\infty} \rightarrow \rho_{0}-\rho^{\infty}$ in $H^{m}$ and $g^{k} \rightarrow g$ in $L^{2}\left(0, T ; H^{m}\right)$ as $k \rightarrow \infty$.

For a.e. $t \in(0, T)$, let $w^{k}=w^{k}(t) \in D_{0}^{1}$ be the unique weak solution to the elliptic boundary value problem

$$
\Delta w^{k}=\operatorname{div} g^{k} \quad \text { in } \quad \Omega \quad \text { and } \quad w^{k}=0 \quad \text { on } \quad \partial \Omega .
$$

It is obvious that $w^{k} \in L^{2}\left(0, T ; D_{0}^{1}\right)$ and $\left|w^{k}(t)-v(t)\right|_{D_{0}^{1}} \leq\left|g^{k}(t)-g(t)\right|_{L^{2}}$ for a.e. $t \in(0, T)$. Then by virtue of the elliptic regularity result in [3], we deduce that $w^{k} \in L^{2}\left(0, T ; D_{0}^{1} \cap D^{m+3}\right)$ and

$$
\begin{aligned}
\left|w^{k}(t)-v(t)\right|_{D_{0}^{1} \cap D^{m+1}} & \leq C\left(\left|\operatorname{div} g^{k}(t)-\operatorname{div} g(t)\right|_{H^{m-1}}+\left|w^{k}(t)-v(t)\right|_{D_{0}^{1}}\right) \\
& \leq C\left|g^{k}(t)-g(t)\right|_{H^{m}}
\end{aligned}
$$

for a.e. $t \in(0, T)$. Hence it follows that $w^{k} \rightarrow v$ in $L^{2}\left(0, T ; D_{0}^{1} \cap D^{m+1}\right)$ as $k \rightarrow \infty$. Therefore, recalling that $C^{\infty}\left([0, T] ; D_{0}^{1} \cap D^{m+3}\right)$ is dense in $L^{2}\left(0, T ; D_{0}^{1} \cap D^{m+3}\right)$, we conclude that there exists a sequence $\left\{v^{k}\right\}$ in $C^{\infty}\left([0, T] ; D_{0}^{1} \cap D^{m+3}\right)$ such that $v^{k} \rightarrow v$ in $L^{2}\left(0, T ; D_{0}^{1} \cap D^{m+1}\right)$ as $k \rightarrow \infty$. In view of the Sobolev embedding results

$$
H^{m+3} \hookrightarrow C^{m+1}(\bar{\Omega}) \text { and } D_{0}^{1} \cap D^{m+3} \hookrightarrow C^{m+1}(\bar{\Omega}),
$$

we complete the proof of (2.4). To treat the case of unbounded domains, we also need a cut-off procedure. Assuming that $\Omega$ is an unbounded domain such as the whole space, the half space and an exterior domain, we choose a sufficiently large integer $R_{0}>1$ so that

$$
\mathbf{R}^{3} \backslash \Omega \subset B_{R_{0} / 2} \quad \text { if } \quad \mathbf{R}^{3} \backslash \Omega \subset \subset \mathbf{R}^{3},
$$

where for each $R>0, B_{R}$ denotes the open ball of radius $R$ centered at the origin: $B_{R}=\left\{x \in \mathbf{R}^{3}:|x|<R\right\}$. Then taking a cut-off function $\varphi \in C_{c}^{\infty}\left(B_{1}\right)$ such that $\varphi=1$ in $B_{1 / 2}$, we define $\rho_{0}^{R}$ and $v^{R}$ by

$$
\rho_{0}^{R}(x)=\rho^{\infty}+\varphi(x / R)\left(\rho_{0}(x)-\rho^{\infty}\right) \quad \text { and } \quad v^{R}(t, x)=\varphi(x / R) v(t, x)
$$

for $(t, x) \in[0, T] \times \Omega$ and $R>R_{0}$. Note that $\rho_{0}^{R}=\rho^{\infty}$ and $v^{R}=0$ in $(0, T) \times$ $\left(\Omega \backslash \Omega_{R}\right)$, where $\Omega_{R}=\Omega \cap B_{R}$. Moreover, it is easy to show that

$$
\left|\rho_{0}^{R}-\rho_{0}\right|_{H^{m}}+\left|v^{R}-v\right|_{L^{2}\left(0, T ; D_{0}^{1} \cap D^{m+1}\right)} \rightarrow 0 \quad \text { as } \quad R \rightarrow \infty .
$$


Hence applying this cut-off technique to $\rho_{0}^{k}$ and $v^{k}$ for each $k \geq 1$, we may assume without loss of generality that if $\Omega$ is an unbounded domain, then

$$
\rho_{0}^{k}(x)=\rho^{\infty} \quad \text { and } \quad v^{k}(t, x)=0 \quad \text { for } \quad t \in[0, T], x \in \Omega \backslash \Omega_{R_{k}},
$$

where $\left\{R_{k}\right\}$ is a sequence such that $R_{0}<R_{1}<R_{2}<\cdots$ and $R_{k} \rightarrow \infty$.

Now we consider the following regularized problem

$$
\rho_{t}+v^{k} \cdot \nabla \rho+\rho \operatorname{div} v^{k}=0 \quad \text { in } \quad(0, T) \times \Omega \quad \text { and } \quad \rho(0)=\rho_{0}^{k} \quad \text { in } \quad \Omega
$$

for each $k \geq 1$. Then since $\rho_{0}^{k} \in C^{m+1}(\bar{\Omega}), v^{k} \in C^{m+1}([0, T] \times \bar{\Omega})$ and $v^{k}=0$ on $[0, T] \times \partial \Omega$, it follows from the standard hyperbolic theory that there exists a unique solution $\rho^{k} \in C^{m+1}([0, T] \times \bar{\Omega})$ to the problem $(2.6)$ and the solution $\rho^{k}$ can be represented by

$$
\rho^{k}(t, x)=\rho_{0}^{k}\left(U^{k}(0, t, x)\right) \exp \left[-\int_{0}^{t} \operatorname{div} v^{k}\left(s, U^{k}(s, t, x)\right) d s\right],
$$

where $U^{k} \in C^{m+1}([0, T] \times[0, T] \times \bar{\Omega})$ is the solution to the initial value problem

$$
\left\{\begin{aligned}
\frac{\partial}{\partial t} U^{k}(t, s, x) & =v^{k}\left(t, U^{k}(t, s, x)\right), \quad 0 \leq t \leq T \\
U^{k}(s, s, x) & =x, \quad 0 \leq s \leq T, \quad x \in \bar{\Omega} .
\end{aligned}\right.
$$

It should be noted from (2.5) that if $\Omega$ is an unbounded domain, then

$$
U^{k}(t, s, x)=x \quad \text { and } \quad \rho^{k}(t, x)=\rho^{\infty} \quad \text { for } \quad t, s \in[0, T], x \in \Omega \backslash \Omega_{R_{k}} .
$$

We will prove that the sequence $\left\{\rho^{k}\right\}$ converges to a solution of the original problem. To show this, we first observe that

$$
\begin{aligned}
& \left|U^{k}(t, s, x)-U^{l}(t, s, x)\right| \\
& \quad \leq \int_{s}^{t}\left|v^{k}\left(\tau, U^{k}(\tau, s, x)\right)-v^{l}\left(\tau, U^{l}(\tau, s, x)\right)\right| d \tau \\
& \quad \leq \int_{s}^{t}\left|v^{k}(\tau)-v^{l}(\tau)\right|_{L^{\infty}} d \tau+\int_{s}^{t}\left|\nabla v^{l}(\tau)\right|_{L^{\infty}}\left|U^{k}(\tau, s, x)-U^{l}(\tau, s, x)\right| d \tau .
\end{aligned}
$$

Then in view of Gronwall's inequality, we have

$$
\begin{aligned}
& \left|U^{k}(t, s, x)-U^{l}(t, s, x)\right| \\
& \quad \leq\left(\int_{0}^{T}\left|v^{k}(\tau)-v^{l}(\tau)\right|_{L^{\infty}} d \tau\right) \exp \left(\int_{0}^{T}\left|\nabla v^{l}(\tau)\right|_{L^{\infty}} d \tau\right) \\
& \quad \leq C\left(\int_{0}^{T}\left|v^{k}(\tau)-v^{l}(\tau)\right|_{D_{0}^{1} \cap D^{2}} d \tau\right) \exp \left(C \int_{0}^{T}\left|v^{l}(\tau)\right|_{D_{0}^{1} \cap D^{3}} d \tau\right)
\end{aligned}
$$

for each $s, t \in[0, T]$ and $x \in \bar{\Omega}$, and thus

$$
\left|U^{k}-U^{l}\right|_{C([0, T] \times[0, T] \times \bar{\Omega})} \rightarrow 0 \quad \text { as } \quad k, l \rightarrow \infty .
$$

Hence it follows from the well-known embedding result $H^{2} \hookrightarrow C^{0, \frac{1}{2}}$ that

$$
\begin{aligned}
& \int_{0}^{T}\left|\operatorname{div} v\left(s, U^{k}(s, t, x)\right)-\operatorname{div} v\left(s, U^{l}(s, t, x)\right)\right| d s \\
& \quad \leq C \int_{0}^{T}|\nabla v(s)|_{H^{2}}\left|U^{k}(s, t, x)-U^{l}(s, t, x)\right|^{\frac{1}{2}} d s \rightarrow 0 \quad \text { as } \quad k, l \rightarrow \infty
\end{aligned}
$$


uniformly in $(t, x) \in[0, T] \times \bar{\Omega}$. Therefore, observing that

$$
\begin{aligned}
& \int_{0}^{t}\left|\operatorname{div} v^{k}\left(s, U^{k}(s, t, x)\right)-\operatorname{div} v^{l}\left(s, U^{l}(s, t, x)\right)\right| d s \\
& \leq \int_{0}^{T}\left(\left|\operatorname{div} v^{k}(s)-\operatorname{div} v(s)\right|_{L^{\infty}}+\left|\operatorname{div} v^{l}(s)-\operatorname{div} v(s)\right|_{L^{\infty}}\right) d s \\
& \quad+\int_{0}^{T}\left|\operatorname{div} v\left(s, U^{k}(s, t, x)\right)-\operatorname{div} v\left(s, U^{l}(s, t, x)\right)\right| d s,
\end{aligned}
$$

we deduce from (2.7) that

$$
\left|\rho^{k}-\rho^{l}\right|_{C([0, T] \times \bar{\Omega})} \rightarrow 0 \quad \text { as } \quad k, l \rightarrow \infty .
$$

This proves the existence of a limit $\rho$ in $C([0, T] \times \bar{\Omega})$ such that

$$
\rho^{k} \rightarrow \rho \quad \text { in } \quad C([0, T] \times \bar{\Omega}) \quad \text { as } \quad k \rightarrow \infty .
$$

It is easy to show that $\rho$ is a weak solution to the original problem (2.1).

To prove the higher regularity of $\rho$, we derive uniform estimates for $\rho^{k}$ in higher norms. Multiplying the equation in (2.6) with $\rho=\rho^{k}$ by $\rho^{k}-\rho^{\infty}$ and integrating over $\Omega$, we have

$$
\frac{d}{d t} \int\left|\rho^{k}-\rho^{\infty}\right|^{2} d x \leq C \int\left|\operatorname{div} v^{k}\right|\left(\left|\rho^{k}-\rho^{\infty}\right|+\rho^{\infty}\right)\left|\rho-\rho^{\infty}\right| d x
$$

and thus

$$
\frac{d}{d t}\left|\rho^{k}-\rho^{\infty}\right|_{L^{2}}^{2} \leq C\left|\nabla v^{k}\right|_{L^{\infty}}\left|\rho^{k}-\rho^{\infty}\right|_{L^{2}}^{2}+C \rho^{\infty}\left|\rho^{k}-\rho^{\infty}\right|_{L^{2}}\left|\nabla v^{k}\right|_{L^{2}}
$$

Let $\alpha$ be a multi-index with $1 \leq|\alpha|=\alpha_{1}+\alpha_{2}+\alpha_{3} \leq m$. Then taking the differential operator $D^{\alpha}$ to $(2.6)$, we have

$$
\begin{aligned}
& \left(D^{\alpha} \rho^{k}\right)_{t}+v^{k} \cdot \nabla\left(D^{\alpha} \rho^{k}\right) \\
& \quad=v^{k} \cdot \nabla\left(D^{\alpha} \rho^{k}\right)-D^{\alpha}\left(v^{k} \cdot \nabla \rho^{k}\right)-D^{\alpha}\left(\rho^{k} \operatorname{div} v^{k}\right) \equiv F_{\alpha}^{k} .
\end{aligned}
$$

Multiplying this by $D^{\alpha} \rho$ and integrating over $\Omega$, we obtain

$$
\frac{d}{d t} \int\left|D^{\alpha} \rho^{k}\right|^{2} d x \leq C \int\left(\left|\operatorname{div} v^{k}\right|\left|D^{\alpha} \rho^{k}\right|^{2}+\left|F_{\alpha}^{k}\right|\left|D^{\alpha} \rho^{k}\right|\right) d x
$$

and thus

$$
\frac{d}{d t}\left|D^{\alpha} \rho^{k}\right|_{L^{2}}^{2} \leq C\left(\left|\operatorname{div} v^{k}\right|_{L^{\infty}}\left|D^{\alpha} \rho^{k}\right|_{L^{2}}^{2}+\left|F_{\alpha}^{k}\right|_{L^{2}}\left|D^{\alpha} \rho^{k}\right|_{L^{2}}\right) .
$$

But since

$$
\left|v^{k} \cdot \nabla\left(D^{\alpha} \rho^{k}\right)-D^{\alpha}\left(v^{k} \cdot \nabla \rho^{k}\right)\right| \leq C \sum_{l=1}^{|\alpha|}\left|\nabla^{|\alpha|+1-l} v^{k}\right|\left|\nabla^{l} \rho^{k}\right|,
$$

it follows from Hölder and Sobolev inequalities that

$$
\sup _{1 \leq|\alpha| \leq m}\left|v^{k} \cdot \nabla\left(D^{\alpha} \rho^{k}\right)-D^{\alpha}\left(v^{k} \cdot \nabla \rho^{k}\right)\right|_{L^{2}} \leq C\left|v^{k}\right|_{D_{0}^{1} \cap D^{m+1}}\left|\nabla \rho^{k}\right|_{H^{m-1}}
$$

A similar calculation also shows that

$$
\sup _{1 \leq|\alpha| \leq m}\left|D^{\alpha}\left(\rho^{k} \operatorname{div} v^{k}\right)\right|_{L^{2}} \leq C\left|v^{k}\right|_{D_{0}^{1} \cap D^{m+1}}\left(\left|\nabla \rho^{k}\right|_{H^{m-1}}+\left|\rho^{k}\right|_{L^{\infty}}\right) .
$$


Hence from (2.11) and (2.12), it follows that

$$
\frac{d}{d t}\left|\rho^{k}-\rho^{\infty}\right|_{H^{m}}^{2} \leq C\left|v^{k}\right|_{D_{0}^{1} \cap D^{m+1}}\left|\rho^{k}-\rho^{\infty}\right|_{H^{m}}^{2}+C \rho^{\infty}\left|v^{k}\right|_{D_{0}^{1} \cap D^{m+1}}\left|\rho^{k}-\rho^{\infty}\right|_{H^{m}}
$$

Therefore, in view of Gronwall's inequality, we conclude that

$$
\begin{gathered}
\left|\rho^{k}(t)-\rho^{\infty}\right|_{H^{m}} \leq\left(\left|\rho_{0}^{k}-\rho^{\infty}\right|_{H^{m}}+C \rho^{\infty} \int_{0}^{t}\left|v^{k}(s)\right|_{D_{0}^{1} \cap D^{m+1}} d s\right) \\
\times \exp \left(C \int_{0}^{t}\left|v^{k}(s)\right|_{D_{0}^{1} \cap D^{m+1}} d s\right)
\end{gathered}
$$

for each $t \in[0, T]$. As a consequence of (2.10) and (2.13), we deduce that

$$
\rho^{k}-\rho^{\infty} \stackrel{*}{\rightarrow} \rho-\rho^{\infty} \quad \text { in } \quad L^{\infty}\left(0, T ; H^{m}\right) \quad \text { as } \quad k \rightarrow \infty .
$$

Moreover since $\rho_{t}=-\operatorname{div}(\rho v) \in L^{\infty}\left(0, T ; H^{m-1}\right)$, it follows from a classical embedding result (see [26] for instance) that $\rho-\rho^{\infty} \in C\left([0, T] ; H^{m-1}\right) \cap C\left([0, T] ; H^{m}-\right.$ weak). To prove the strong time-continuity of $\rho-\rho^{\infty}$ in $H^{m}$, we observe that for each fixed $t \in[0, T], \rho^{k}(t)-\rho^{\infty} \rightarrow \rho(t)-\rho^{\infty}$ weakly in $H^{m}$. Hence from (2.13), it follows immediately that

$$
\begin{aligned}
\left|\rho(t)-\rho^{\infty}\right|_{H^{m}} \leq\left(\left|\rho_{0}-\rho^{\infty}\right|_{H^{m}}+C \rho^{\infty} \int_{0}^{t}|v(s)|_{D_{0}^{1} \cap D^{m+1}} d s\right) \\
\times \exp \left(C \int_{0}^{t}|v(s)|_{D_{0}^{1} \cap D^{m+1}} d s\right)
\end{aligned}
$$

for each $t \in[0, T]$. In particular, we have

$$
\limsup _{t \rightarrow+0}\left|\rho(t)-\rho^{\infty}\right|_{H^{m}} \leq\left|\rho_{0}-\rho^{\infty}\right|_{H^{m}}
$$

which implies that $\rho-\rho^{\infty}$ is right-continuous in $H^{m}$ at $t=0$. Since the equation in (2.1) is invariant under the reflections and translations in time, we conclude that $\rho-\rho^{\infty} \in C\left([0, T] ; H^{m}\right)$. It also follows from $(2.1)$ that $\rho_{t} \in C\left([0, T] ; H^{m-1}\right)$. It is easy to prove the uniqueness of solutions in this regularity class. This completes the proof of (i). The estimate in (ii) follows immediately from (2.14). Hence it remains to show (iii). By virtue of the regularity of $v$, we can prove the uniqueness of a solution $U$ in $C([0, T] \times[0, T] \times \bar{\Omega})$ to the problem $(2.3)$, whose existence is guaranteed by (2.8) and (2.9). Finally, from (2.7), (2.9) and (2.10), we obtain the representation formula $(2.2)$ for the solution $\rho$.

2.2. A linear parabolic system. Next, let $\Omega$ be a bounded domain in $\mathbf{R}^{3}$ with smooth boundary, and we consider the following linear parabolic problem

$$
\left\{\begin{aligned}
\rho u_{t}+L u=F & \text { in } \quad(0, T) \times \Omega, \\
u(0)=u_{0} \quad \text { in } \quad \Omega, & u=0 \quad \text { on }(0, T) \times \partial \Omega,
\end{aligned}\right.
$$

where $\rho$ is a known scalar field in $(0, T) \times \Omega$ such that

$$
\rho \in C\left([0, T] ; H^{3}\right), \quad \rho_{t} \in C\left([0, T] ; H^{2}\right) \quad \text { and } \quad \rho \geq \delta \quad \text { on } \quad[0, T] \times \bar{\Omega}
$$


for some constant $\delta>0$. Recall that $L=-\mu \Delta-(\lambda+\mu) \nabla$ div is a strongly elliptic operator (see [3] for instance). Then applying a standard method such as a semidiscrete Galerkin method or the method of continuity, we can prove the following existence and regularity results on solutions to the linear parabolic problem (2.15). See also the papers $[27,28,29]$ for similar results.

Lemma 2.2. (i) Assume that $u_{0} \in H_{0}^{1}$ and $F \in L^{2}\left(0, T ; L^{2}\right)$. Then there exists a unique strong solution $u$ to the problem (2.15) such that

$$
u \in C\left([0, T] ; H_{0}^{1}\right) \cap L^{2}\left(0, T ; H^{2}\right) \quad \text { and } \quad u_{t} \in L^{2}\left(0, T ; L^{2}\right) .
$$

(ii) If $u_{0} \in H_{0}^{1} \cap H^{2}, F \in L^{\infty}\left(0, T ; L^{2}\right)$ and $F_{t} \in L^{2}\left(0, T ; H^{-1}\right)$, then the solution u satisfies

$$
u \in L^{\infty}\left(0, T ; H^{2}\right), \quad u_{t} \in L^{2}\left(0, T ; H_{0}^{1}\right) \quad \text { and } \quad u_{t t} \in L^{2}\left(0, T ; H^{-1}\right) .
$$

(iii) Finally, if $u_{0} \in H_{0}^{1} \cap H^{3}, F \in L^{\infty}\left(0, T ; H^{1}\right), F_{t} \in L^{2}\left(0, T ; L^{2}\right)$ and $u_{t}(0)=$ $\rho(0)^{-1}\left(F(0)-L u_{0}\right) \in H_{0}^{1}$, then the solution $u$ also satisfies

$$
u \in L^{\infty}\left(0, T ; H^{3}\right), \quad u_{t} \in L^{2}\left(0, T ; H^{2}\right) \quad \text { and } \quad u_{t t} \in L^{2}\left(0, T ; L^{2}\right) .
$$

Remark 2.3. Let $u$ be the solution obtained in the result (iii) of Lemma 2.2. Then by virtue of a standard embedding result, we have

$$
u \in C\left([0, T] ; H^{2}\right) \quad \text { and } \quad u_{t} \in C\left([0, T] ; H_{0}^{1}\right) .
$$

Moreover, it follows from an elliptic regularity result that if $F \in L^{2}\left(0, T ; H^{2}\right)$ in addition, then $u$ also satisfies

$$
u \in L^{2}\left(0, T ; H^{4}\right) \quad \text { and so } \quad u \in C\left([0, T] ; H^{3}\right) .
$$

Standard arguments based on Lemma 2.2 enable us to prove the smoothing effect of the solution $u$ for positive time $t>0$, provided that $\rho$ and $F$ are sufficiently regular in $t>0$. Throughout this paper, we denote

$$
L_{l o c}^{r}((0, T] ; X)=\bigcap_{\tau>0} L^{r}(\tau, T ; X)
$$

for $1 \leq r \leq \infty$ and a Banach space $X$.

Lemma 2.4. Let $u_{0} \in H_{0}^{1}$ and $F \in L^{2}\left(0, T ; L^{2}\right)$. Assume in addition to (2.16) that

$$
\begin{gathered}
\rho_{t t} \in L_{l o c}^{\infty}\left((0, T] ; L^{2}\right), \quad \rho_{t t t} \in L_{l o c}^{2}\left((0, T] ; H^{-1}\right), \quad F \in L_{l o c}^{\infty}\left((0, T] ; H^{2}\right), \\
F_{t} \in L_{l o c}^{\infty}\left((0, T] ; H^{1}\right), \quad F_{t t} \in L_{l o c}^{\infty}\left((0, T] ; L^{2}\right) \quad \text { and } \quad F_{t t t} \in L_{l o c}^{2}\left((0, T] ; H^{-1}\right) .
\end{gathered}
$$

Then there exists a unique solution $u$ to the problem (2.15) such that

$$
\begin{gathered}
u \in C\left([0, T] ; H_{0}^{1}\right) \cap L^{2}\left(0, T ; H^{2}\right), \quad u_{t} \in L^{2}\left(0, T ; L^{2}\right) ; \\
u \in L_{l o c}^{\infty}\left((0, T] ; H^{4}\right), \quad u_{t} \in L_{l o c}^{\infty}\left((0, T] ; H_{0}^{1} \cap H^{3}\right), \quad u_{t t} \in L_{l o c}^{\infty}\left((0, T] ; H_{0}^{1} \cap H^{2}\right), \\
u_{t t t} \in L_{l o c}^{\infty}\left((0, T] ; L^{2}\right) \cap L_{l o c}^{2}\left((0, T] ; H_{0}^{1}\right) \quad \text { and } \quad u_{t t t t} \in L_{l o c}^{2}\left((0, T] ; H^{-1}\right) .
\end{gathered}
$$


Proof. The result (i) of Lemma 2.2 guarantees the existence of a unique solution $u$ with the regularity

$$
u \in C\left([0, T] ; H_{0}^{1}\right) \cap L^{2}\left(0, T ; H^{2}\right) \quad \text { and } \quad u_{t} \in L^{2}\left(0, T ; L^{2}\right) .
$$

We prove the additional regularity of $u$ using a standard iterative argument (see [25] for instance). Let $t_{0}$ be a fixed small time in $(0, T)$.

(a) Since $u \in L^{2}\left(0, T ; H_{0}^{1} \cap H^{2}\right)$, we can choose a time $t_{1}$ in $\left(0, t_{0}\right)$ such that $u\left(t_{1}\right) \in H_{0}^{1} \cap H^{2}$. Then the result (ii) yields that $u \in L^{\infty}\left(t_{1}, T ; H_{0}^{1} \cap H^{2}\right)$ and $u_{t} \in L^{2}\left(t_{1}, T ; H_{0}^{1}\right)$. Moreover, since $F \in L^{2}\left(t_{1}, T ; H^{1}\right)$, it follows from the elliptic regularity result that $u \in L^{2}\left(t_{1}, T ; H^{3}\right)$.

(b) There is a time $t_{2} \in\left(t_{1}, t_{0}\right)$ such that $u\left(t_{2}\right) \in H_{0}^{1} \cap H^{3}$ and $u_{t}\left(t_{2}\right) \in H_{0}^{1}$. In view of the result (iii), we deduce that

$$
\left.u \in L^{\infty}\left(t_{2}, T ; H^{3}\right), \quad u_{t} \in L^{2}\left(t_{2}, T ; H^{2}\right) \quad \text { and } \quad u_{t t} \in L^{2}\left(t_{2}, T\right] ; L^{2}\right) .
$$

(c) There is a time $t_{3} \in\left(t_{2}, t_{0}\right)$ such that $u_{t}\left(t_{3}\right) \in H_{0}^{1} \cap H^{2}$. Note that $w=u_{t}$ is the unique solution to the problem

$$
\left\{\begin{array}{c}
\rho w_{t}+L w=G \quad \text { in }\left(t_{3}, T\right) \times \Omega, \\
w\left(t_{3}\right)=u_{t}\left(t_{3}\right) \quad \text { in } \quad \Omega, \quad w=0 \quad \text { on }\left(t_{3}, T\right) \times \partial \Omega,
\end{array}\right.
$$

where $G=F_{t}-\rho_{t} u_{t}$. Note that $G \in L^{2}\left(t_{3}, T ; H^{1}\right)$ and $G_{t} \in L^{2}\left(t_{3}, T ; H^{-1}\right)$. Hence it follows from the result (ii) that

$$
w \in L^{\infty}\left(t_{3}, T ; H^{2}\right), \quad w_{t} \in L^{2}\left(t_{3}, T ; H_{0}^{1}\right) \quad \text { and } \quad w_{t t} \in L^{2}\left(t_{3}, T ; H^{-1}\right) .
$$

Moreover, using the elliptic regularity result again, we deduce that

$$
u \in L^{\infty}\left(t_{3}, T ; H^{4}\right) \text { and } w \in L^{2}\left(t_{3}, T ; H^{3}\right) .
$$

(d) There is $t_{4} \in\left(t_{3}, t_{0}\right)$ such that $w\left(t_{4}\right) \in H_{0}^{1} \cap H^{3}$ and $w_{t}\left(t_{4}\right) \in H_{0}^{1}$. Note that $G=F_{t}-\rho_{t} w \in L^{\infty}\left(t_{4}, T ; H^{1}\right)$ and $G_{t} \in L^{2}\left(t_{4}, T ; L^{2}\right)$. Hence it follows from (iii) that

$$
w \in L^{\infty}\left(t_{4}, T ; H^{3}\right), \quad w_{t} \in L^{2}\left(t_{4}, T ; H^{2}\right) \quad \text { and } \quad w_{t t} \in L^{2}\left(t_{4}, T ; L^{2}\right) .
$$

(e) There is a time $t_{5} \in\left(t_{4}, t_{0}\right)$ such that $w_{t}\left(t_{5}\right) \in H_{0}^{1} \cap H^{2}$ and $v=w_{t}$ is the unique solution to the problem

$$
\left\{\begin{array}{c}
\rho v_{t}+L v=H \quad \text { in }\left(t_{5}, T\right) \times \Omega, \\
w\left(t_{5}\right)=u_{t}\left(t_{5}\right) \quad \text { in } \quad \Omega, \quad w=0 \quad \text { on }\left(t_{5}, T\right) \times \partial \Omega,
\end{array}\right.
$$

where $H=G_{t}-\rho_{t} w_{t}$. Since $H \in L^{\infty}\left(t_{5}, T ; L^{2}\right)$ and $H_{t} \in L^{2}\left(t_{5}, T ; H^{-1}\right)$, it follows from (ii) that

$$
v \in L^{\infty}\left(t_{5}, T ; H^{2}\right), \quad v_{t} \in L^{2}\left(t_{5}, T ; H_{0}^{1}\right) \quad \text { and } \quad v_{t t} \in L^{2}\left(t_{5}, T ; H^{-1}\right) .
$$

Observing that $0<t_{1}<t_{2}<t_{3}<t_{4}<t_{5}<t_{0}$ and $t_{0}$ can be chosen to be arbitrarily small, we complete the proof of Lemma 2.4. 


\section{A priori estimates For the LineARized PROBlem}

To prove Theorem 1.1, we consider the following linearized problem

$$
\begin{aligned}
& \rho_{t}+\operatorname{div}(\rho v)=0 \quad \text { in }(0, T) \times \Omega, \\
& \rho u_{t}+L u+\nabla p=\rho(f-v \cdot \nabla v) \quad \text { in }(0, T) \times \Omega, \\
& \left.(\rho, u)\right|_{t=0}=\left(\rho_{0}, u_{0}\right) \quad \text { in } \quad \Omega, \quad u=0 \quad \text { on }(0, T) \times \partial \Omega, \\
& \rho(t, x) \rightarrow \rho^{\infty}, \quad u(t, x) \rightarrow 0 \quad \text { as } \quad|x| \rightarrow \infty, \quad(t, x) \in(0, T) \times \Omega,
\end{aligned}
$$

where $v$ is a known vector field in $(0, T) \times \Omega$ such that

$$
v \in C\left([0, T] ; D_{0}^{1} \cap D^{3}\right) \cap L^{2}\left(0, T ; D^{4}\right), \quad v_{t} \in L^{\infty}\left(0, T ; D_{0}^{1}\right) \cap L^{2}\left(0, T ; D^{2}\right) .
$$

Recall again that $L u=-\mu \Delta u-(\lambda+\mu) \nabla \operatorname{div} u$ and $p=p(\rho)$.

First, from the lemmas in Section 2, we obtain an existence result for positive initial densities.

Lemma 3.1. Let $\Omega$ be a bounded domain in $\mathbf{R}^{3}$ with smooth boundary. In addition to (1.9) and (3.5), we assume that $\rho_{0} \geq \delta$ in $\Omega$ for some constant $\delta>0$ and $f(0)-v(0) \cdot \nabla v(0)-\rho_{0}^{-1}\left(L u_{0}+\nabla p\left(\rho_{0}\right)\right) \in H_{0}^{1}$. Then there exists a unique solution $(\rho, u)$ to the linearized problem (3.1), (3.2) and (3.3) such that

$$
\begin{gathered}
\rho \in C\left([0, T] ; H^{3}\right), \quad \rho_{t} \in C\left([0, T] ; H^{2}\right), \\
u \in C\left([0, T] ; H_{0}^{1} \cap H^{3}\right) \cap L^{2}\left(0, T ; H^{4}\right), \\
u_{t} \in C\left([0, T] ; H_{0}^{1}\right) \cap L^{2}\left(0, T ; H^{2}\right), \\
u_{t t} \in L^{2}\left(0, T ; L^{2}\right) \quad \text { and } \quad \rho \geq \underline{\delta} \quad \text { on } \quad[0, T] \times \bar{\Omega}
\end{gathered}
$$

for some constant $\underline{\delta}>0$.

Proof. The existence and regularity of a unique solution $\rho$ to the linear hyperbolic problem (3.1) and (3.3) were already proved in Lemma 2.1. To prove the remaining part of the lemma, let us define $F$ by $F=-\nabla p(\rho)+\rho(f-v \cdot \nabla v)$. Then by virtue of (1.9), (3.5) and the regularity of $\rho$, we can easily show that $F \in L^{2}\left(0, T ; H^{2}\right)$ and $F_{t} \in L^{2}\left(0, T ; L^{2}\right)$. Moreover since $\rho_{0}^{-1}\left(F(0)-L u_{0}\right) \in H_{0}^{1}$, Lemma 2.2 and Remark 2.3 allow us to deduce the existence and regularity of a unique solution $u$ to the linear parabolic problem (3.2) and (3.3). This completes the proof of Lemma 3.1 .

Assume that $\rho_{0}, u_{0}, v, f, p=p(\cdot)$ and $\Omega$ satisfy the hypotheses of Lemma 3.1. Then it follows from Lemma 3.1 that there exists a unique strong solution $(\rho, u)$ to the linear problem (3.1), (3.2) and (3.3) satisfying the regularity (3.6). The purpose of this section is to derive some local (in time) a priori estimates for $(\rho, u)$ which are independent of the lower bound $\delta$ of $\rho_{0}$ and size of the domain $\Omega$. Let us choose a constant $c_{0}>1$ so that

$$
1+\rho^{\infty}+\left|\rho_{0}-\rho^{\infty}\right|_{H^{3}}+\left|u_{0}\right|_{D_{0}^{1}}+\left|\sqrt{\rho}_{0} g_{2}\right|_{L^{2}}+\left|g_{2}\right|_{D_{0}^{1}}<c_{0},
$$


where $g_{2}=\rho_{0}^{-1}\left(L u_{0}+\nabla p\left(\rho_{0}\right)\right)-f(0)=-v(0) \cdot \nabla v(0)-u_{t}(0)$, and assume that

$$
\begin{array}{r}
|v(0)|_{D_{0}^{1} \cap D^{3}} \leq 1+c_{1}, \\
\sup _{0 \leq t \leq T_{*}}|v(t)|_{D_{0}^{1}}+\int_{0}^{T_{*}}|v(t)|_{D^{2}}^{2} d t \leq 1+c_{2}, \\
\sup _{0 \leq t \leq T_{*}}|v(t)|_{D^{2}}+\int_{0}^{T_{*}}\left(\left|v_{t}(t)\right|_{D_{0}^{1}}^{2}+|v(t)|_{D^{3}}^{2}\right) d t \leq 1+c_{3}, \\
\operatorname{ess~sup}_{0<t<T_{*}}\left(\left|v_{t}(t)\right|_{D_{0}^{1}}+|v(t)|_{D^{3}}\right)+\int_{0}^{T_{*}}\left(\left|v_{t}(t)\right|_{D^{2}}^{2}+|v(t)|_{D^{4}}^{2}\right) d t \leq 1+c_{4}
\end{array}
$$

for some time $T_{*} \in(0, T)$ and constants $c_{i}$ 's with $1<c_{0} \leq c_{1} \leq c_{2} \leq c_{3} \leq c_{4}$. The constants $c_{i}$ 's, $1 \leq i \leq 4$, and $T_{*}$ will be determined later and depend only on $c_{0}$ and the parameters of $C$. Throughout this and next two sections, we denote by $C$ a generic positive constant depending only on the fixed constants $\mu, \lambda, T,|p|_{C^{3}\left(\overline{\mathbf{R}}_{+}\right)}$ and the norm of $f$. Moreover, $M=M(\cdot)$ denotes an increasing continuous function from $[1, \infty)$ to $[1, \infty)$ which is independent of $\delta$ and the size of $\Omega$.

\section{Lemma 3.2.}

$$
\begin{gathered}
|\rho(t)|_{L^{\infty}}+\left|\rho(t)-\rho^{\infty}\right|_{H^{3}} \leq C c_{0}, \quad\left|p(t)-p^{\infty}\right|_{H^{3}} \leq M\left(c_{0}\right), \quad\left|\rho_{t}(t)\right|_{H^{1}} \leq C c_{3}^{2}, \\
\left|p_{t}(t)\right|_{H^{1}} \leq M\left(c_{0}\right) c_{3}^{2}, \quad \int_{0}^{t}\left|\rho_{t t}(s)\right|_{L^{2}}^{2} d s \leq C c_{3}^{8}, \quad \int_{0}^{t}\left|p_{t t}(s)\right|_{L^{2}}^{2} d s \leq M\left(c_{0}\right) c_{3}^{8}, \\
\left|\rho_{t}(t)\right|_{H^{2}} \leq C c_{4}^{2}, \quad\left|p_{t}(t)\right|_{H^{2}} \leq M\left(c_{0}\right) c_{4}^{2} \quad \text { and } \quad \inf _{\Omega} \rho(t) \geq C^{-1} \delta
\end{gathered}
$$

for $0 \leq t \leq \min \left(T_{*}, T_{1}\right)$, where $T_{1}=\left(1+c_{4}\right)^{-1}$ and $p^{\infty}=p\left(\rho^{\infty}\right)$.

Proof. From Lemma 2.1, we recall that

$$
\left|\rho(t)-\rho^{\infty}\right|_{H^{3}} \leq\left(\left|\rho_{0}-\rho^{\infty}\right|_{H^{3}}+\rho^{\infty}\right) \exp \left(C \int_{0}^{t}|v(s)|_{D_{0}^{1} \cap D^{4}} d s\right)
$$

and

$$
\inf _{\Omega} \rho(t) \geq\left(\inf _{\Omega} \rho_{0}\right) \exp \left(-C \int_{0}^{t}|v(s)|_{D_{0}^{1} \cap D^{4}} d s\right)
$$

for $0 \leq t \leq T$. Hence observing that

$$
\int_{0}^{t}|v(s)|_{D_{0}^{1} \cap D^{4}} d s \leq t^{\frac{1}{2}}\left(\int_{0}^{t}|v(s)|_{D_{0}^{1} \cap D^{4}}^{2} d s\right)^{\frac{1}{2}} \leq C\left(1+c_{4}\right) t+C\left(\left(1+c_{4}\right) t\right)^{\frac{1}{2}},
$$

we obtain the desired estimate for $\rho$. Then the esimates for $\rho_{t}, \rho_{t t}, p, p_{t}$ and $p_{t t}$ follow immediately from the quations $\rho_{t}=-\operatorname{div}(\rho v)$ and $p=p(\rho)$.

\section{Lemma 3.3.}

$$
|u(t)|_{D_{0}^{1}}^{2}+\int_{0}^{t}|u(s)|_{D^{2}}^{2} d s \leq M\left(c_{0}\right)
$$

for $0 \leq t \leq \min \left(T_{*}, T_{2}\right)$, where $T_{2}=\left(1+c_{4}\right)^{-4}<T_{1}$. 
Proof. Multiplying the equation (3.2) by $u_{t}$ and integrating over $\Omega$, we obtain

$$
\begin{aligned}
\int \rho\left|u_{t}\right|^{2} d x & +\frac{1}{2} \frac{d}{d t} \int \mu|\nabla u|^{2}+(\lambda+\mu)(\operatorname{div} u)^{2} d x \\
= & -\int \nabla p \cdot u_{t} d x+\int \rho(f-v \cdot \nabla v) \cdot u_{t} d x
\end{aligned}
$$

Using Lemma 3.2 together with (3.7), we can estimate the second term of the right hand side in (3.8) as follows:

$$
\begin{aligned}
\int \rho(f-v \cdot \nabla v) \cdot u_{t} d x & \leq|\rho|_{L^{\infty}}^{\frac{1}{2}}|f-v \cdot \nabla v|_{L^{2}}\left|\sqrt{\rho} u_{t}\right|_{L^{2}} \\
& \leq C|\rho|_{L^{\infty}}\left(|f|_{L^{2}}^{2}+|v|_{D_{0}^{1} \cap D^{2}}^{4}\right)+\frac{1}{2}\left|\sqrt{\rho} u_{t}\right|_{L^{2}}^{2} \\
& \leq C c_{0} c_{3}^{4}+\frac{1}{2}\left|\sqrt{\rho} u_{t}\right|_{L^{2}}^{2}
\end{aligned}
$$

To estimate the first term, we observe that

$$
\begin{gathered}
-\int \nabla p \cdot u_{t} d x=\int\left(p-p^{\infty}\right) \operatorname{div} u_{t} d x \\
=\frac{d}{d t} \int\left(p-p^{\infty}\right) \operatorname{div} u d x-\int p_{t} \operatorname{div} u d x \\
\int\left(p-p^{\infty}\right) \operatorname{div} u d x \leq C\left|p(\rho)-p^{\infty}\right|_{L^{2}}^{2}+\frac{\mu}{4}|\nabla u|_{L^{2}}^{2} \leq M\left(c_{0}\right)+\frac{\mu}{4}|\nabla u|_{L^{2}}^{2}
\end{gathered}
$$

and

$$
-\int p_{t} \operatorname{div} u d x \leq\left|p_{t}\right|_{L^{2}}^{2}+|\nabla u|_{L^{2}}^{2} \leq M\left(c_{0}\right) c_{3}^{4}+|\nabla u|_{L^{2}}^{2}
$$

Hence integrating (3.8) in time over $(0, t)$, we have

$$
\begin{aligned}
& \int_{0}^{t}\left|\sqrt{\rho} u_{t}(s)\right|_{L^{2}}^{2} d s+|\nabla u(t)|_{L^{2}}^{2} \\
& \quad \leq M\left(c_{0}\right)\left(1+\left|\nabla u_{0}\right|_{L^{2}}^{2}\right)+M\left(c_{0}\right) c_{3}^{4} t+C \int_{0}^{t}|\nabla u(s)|_{L^{2}}^{2} d s
\end{aligned}
$$

for $0 \leq t \leq \min \left(T_{*}, T_{1}\right)$. Therefore, in view of Gronwall's inequality, we conclude that

$$
\int_{0}^{t}\left|\sqrt{\rho} u_{t}(s)\right|_{L^{2}}^{2} d s+|\nabla u(t)|_{L^{2}}^{2} \leq M\left(c_{0}\right) \quad \text { for } \quad 0 \leq t \leq \min \left(T_{*}, T_{2}\right),
$$

where $T_{2}=\left(1+c_{3}\right)^{-4}<T_{1}$. Moreover, since for each $t \in(0, T), u=u(t) \in D_{0}^{1} \cap D^{2}$ is a solution of the elliptic system

$$
L u=-\nabla p+\rho(f-v \cdot \nabla v)-\rho u_{t} \quad \text { in } \Omega,
$$

it follows from the elliptic regularity result in [3] that

$$
\begin{aligned}
|u|_{D^{2}} & \leq C\left(\left|-\nabla p+\rho(f-v \cdot \nabla v)-\rho u_{t}\right|_{L^{2}}+|u|_{D_{0}^{1}}\right) \\
& \leq M\left(c_{0}\right)\left(1+c_{3}^{2}+\left|\sqrt{\rho} u_{t}\right|_{L^{2}}\right)
\end{aligned}
$$

and thus

$$
\int_{0}^{t}|u(s)|_{D^{2}}^{2} d s \leq M\left(c_{0}\right) \quad \text { for } \quad 0 \leq t \leq \min \left(T_{*}, T_{2}\right)
$$

This completes the proof of Lemma 3.3. 


\section{Lemma 3.4.}

$$
\left|\sqrt{\rho} u_{t}(t)\right|_{L^{2}}^{2}+|u(t)|_{D^{2}}^{2}+\int_{0}^{t}\left(\left|u_{t}(s)\right|_{D_{0}^{1}}^{2}+|u(s)|_{D^{3}}^{2}\right) d s \leq M\left(c_{1}\right) c_{2}^{\frac{3}{2}} c_{3}^{\frac{1}{2}}
$$

for $0 \leq t \leq \min \left(T_{*}, T_{3}\right)$, where $T_{3}=\left(1+c_{4}\right)^{-9}<T_{2}$.

Proof. We differentiate (3.2) with respect to $t$ and have

$$
\rho u_{t t}+L u_{t}+\nabla p_{t}=\rho(f-v \cdot \nabla v)_{t}+\rho_{t}\left(f-v \cdot \nabla v-u_{t}\right) .
$$

Multiplying this by $u_{t}$ and integrating over $\Omega$, we obtain

$$
\begin{aligned}
\frac{1}{2} & \frac{d}{d t} \int \rho\left|u_{t}\right|^{2} d x+\int \mu\left|\nabla u_{t}\right|^{2}+(\lambda+\mu)\left(\operatorname{div} u_{t}\right)^{2} d x \\
\quad & =\int\left(-\nabla p_{t}+\rho(f-v \cdot \nabla v)_{t}+\rho_{t}\left(f-v \cdot \nabla v-\frac{1}{2} u_{t}\right)\right) \cdot u_{t} d x .
\end{aligned}
$$

To estimate each term in the right hand side of (3.10), we follow the arguments in $[2,3,4]$; we first apply the standard inequalities such as Hölder, Sobolev and Young's inequalities and then use Lemma 3.2.

$$
\begin{aligned}
& -\int \nabla p_{t} \cdot u_{t} d x=\int p_{t} \operatorname{div} u_{t} d x \leq C\left|p_{t}\right|_{L^{2}}^{2}+\frac{\mu}{8}\left|\nabla u_{t}\right|_{L^{2}}^{2} \leq M\left(c_{0}\right) c_{3}^{4}+\frac{\mu}{8}\left|\nabla u_{t}\right|_{L^{2}}^{2}, \\
& \int \rho f_{t} \cdot u_{t} d x \leq\left|f_{t}\right|_{L^{2}}|\rho|_{L^{\infty}}^{\frac{1}{2}}\left|\sqrt{\rho} u_{t}\right|_{L^{2}} \leq\left|f_{t}\right|_{L^{2}}^{2}+C c_{0}\left|\sqrt{\rho} u_{t^{2}}\right|_{L^{2}}^{2}, \\
& -\int \rho(v \cdot \nabla v)_{t} \cdot u_{t} d x \leq C|\rho|_{L^{\infty}}^{\frac{1}{2}}\left|v_{t}\right|_{D_{0}^{1}}|v|_{D_{0}^{1}}\left|\sqrt{\rho} u_{t}\right|_{L^{3}} \\
& \leq C|\rho|_{L^{\infty}}^{\frac{3}{4}}\left|v_{t}\right|_{D_{0}^{1}}|v|_{D_{0}^{1}}\left|\sqrt{\rho} u_{t}\right|_{L^{2}}^{\frac{1}{2}}\left|\nabla u_{t}\right|_{L^{2}}^{\frac{1}{2}} \\
& \leq \eta^{-2} C|\rho|_{L^{\infty}}^{3}|v|_{D_{0}^{1}}^{4}\left|\sqrt{\rho} u_{t}\right|_{L^{2}}^{2}+\eta\left|v_{t}\right|_{D_{0}^{1}}^{2}+\frac{\mu}{8}\left|\nabla u_{t}\right|_{L^{2}}^{2} \\
& \leq \eta^{-2} C c_{2}^{7}\left|\sqrt{\rho} u_{t}\right|_{L^{2}}^{2}+\eta\left|v_{t}\right|_{D_{0}^{1}}^{2}+\frac{\mu}{8}\left|\nabla u_{t}\right|_{L^{2}}^{2} \\
& \int \rho_{t}(f-v \cdot \nabla v) \cdot u_{t} d x \leq C\left|\rho_{t}\right|_{H^{1}}\left(|f|_{L^{2}}+|v|_{D_{0}^{1} \cap D^{2}}^{2}\right)\left|\nabla u_{t}\right|_{L^{2}} \\
& \leq C c_{3}^{4}\left(|f|_{L^{2}}^{2}+c_{3}^{4}\right)+\frac{\mu}{8}\left|\nabla u_{t}\right|_{L^{2}}^{2} \\
& \leq C c_{3}^{8}+\frac{\mu}{8}\left|\nabla u_{t}\right|_{L^{2}}^{2}
\end{aligned}
$$

and finally

$$
\begin{aligned}
-\int \rho_{t}\left(\frac{1}{2}\left|u_{t}\right|^{2}\right) d x & =\int \operatorname{div}(\rho v)\left(\frac{1}{2}\left|u_{t}\right|^{2}\right) d x \\
& \leq \int \rho|v|\left|u_{t}\right|\left|\nabla u_{t}\right| d x \leq C|\rho|_{L^{\infty}}^{\frac{3}{4}}|v|_{D_{0}^{1}}\left|\sqrt{\rho} u_{t}\right|_{L^{2}}^{\frac{1}{2}}\left|\nabla u_{t}\right|_{L^{2}}^{\frac{3}{2}} \\
& \leq C c_{2}^{7}\left|\sqrt{\rho} u_{t}\right|_{L^{2}}^{2}+\frac{\mu}{8}\left|\nabla u_{t}\right|_{L^{2}}^{2} .
\end{aligned}
$$


Here $\eta \in(0,1)$ is a small number. Substituting these estimates into (3.10) and taking $\eta=\left(1+c_{3}\right)^{-1}$, we have

$$
\begin{aligned}
& \frac{d}{d t} \int \rho\left|u_{t}\right|^{2} d x+\mu \int\left|\nabla u_{t}\right|^{2} d x \\
& \quad \leq M\left(c_{0}\right)\left(\left|f_{t}\right|_{L^{2}}^{2}+c_{3}^{8}\right)+C c_{3}^{9}\left|\sqrt{\rho} u_{t}\right|_{L^{2}}^{2}+\left(1+c_{3}\right)^{-1}\left|v_{t}\right|_{D_{0}^{1}}^{2}
\end{aligned}
$$

for $0 \leq t \leq \min \left(T_{*}, T_{2}\right)$. On the other hand, since

$$
u_{t} \in C\left([0, T] ; H_{0}^{1}\right) \quad \text { and } \quad u_{t}(0)=-v(0) \cdot \nabla v(0)-g_{2},
$$

it follows that

$$
\left|\sqrt{\rho} u_{t}(0)\right|_{L^{2}}+\left|u_{t}(0)\right|_{D_{0}^{1}} \leq C c_{1}^{3} .
$$

Hence integrating $(3.11)$ over $(0, t)$, we also have

$$
\left|\sqrt{\rho} u_{t}(t)\right|_{L^{2}}^{2}+\int_{0}^{t}\left|\nabla u_{t}(s)\right|_{L^{2}}^{2} d s \leq M\left(c_{1}\right)\left(1+c_{3}^{8} t\right)+C c_{3}^{9} \int_{0}^{t}\left|\sqrt{\rho} u_{t}(s)\right|_{L^{2}}^{2} d s .
$$

Therefore, in view of Gronwall's inequality, we conclude that

$$
\left|\sqrt{\rho} u_{t}(t)\right|_{L^{2}}^{2}+\int_{0}^{t}\left|u_{t}(s)\right|_{D_{0}^{1}}^{2} d s \leq M\left(c_{1}\right) \quad \text { for } \quad 0 \leq t \leq \min \left(T_{*}, T_{3}\right),
$$

where $T_{3}=\left(1+c_{4}\right)^{-9}<T_{2}$. Moreover, since for each $t \in(0, T), u=u(t) \in D_{0}^{1} \cap D^{3}$ is a solution of the elliptic system

$$
L u=-\nabla p+\rho(f-v \cdot \nabla v)-\rho u_{t} \quad \text { in } \quad \Omega,
$$

it follows from the elliptic regularity result in [3] that

$$
\begin{aligned}
|u(t)|_{D^{2}} & \leq M\left(c_{1}\right)\left(1+|v \cdot \nabla v|_{L^{2}}\right) \\
& \leq M\left(c_{1}\right)\left(1+|v|_{D_{0}^{1}}^{\frac{3}{2}}|v|_{D_{0}^{1} \cap D^{2}}^{\frac{1}{2}}\right) \leq M\left(c_{1}\right) c_{2}^{\frac{3}{2}} c_{3}^{\frac{1}{2}}
\end{aligned}
$$

and

$$
\int_{0}^{t}|u(s)|_{D^{3}}^{2} d s \leq M\left(c_{1}\right) \int_{0}^{t}\left(1+|v(s)|_{D_{0}^{1} \cap D^{2}}^{4}+\left|u_{t}(s)\right|_{D_{0}^{1}}^{2}\right) d s \leq M\left(c_{1}\right)
$$

for $0 \leq t \leq \min \left(T_{*}, T_{3}\right)$. This completes the proof of Lemma 3.4.

\section{Lemma 3.5.}

$$
\left|u_{t}(t)\right|_{D_{0}^{1}}^{2}+|u(t)|_{D^{3}}^{2}+\int_{0}^{t}\left(\left|\sqrt{\rho} u_{t t}(s)\right|_{L^{2}}^{2}+\left|u_{t}(s)\right|_{D^{2}}^{2}+|u(s)|_{D^{4}}^{2}\right) d s \leq M\left(c_{1}\right) c_{3}^{12}
$$

for $0 \leq t \leq \min \left(T_{*}, T_{3}\right)$.

Proof. Multiplying (3.9) by $u_{t t}$ and integrating over $\Omega$, we have

$$
\begin{aligned}
& \int \rho\left|u_{t t}\right|^{2} d x+\frac{1}{2} \frac{d}{d t} \int \mu\left|\nabla u_{t}\right|^{2}+(\lambda+\mu)\left(\operatorname{div} u_{t}\right)^{2} d x \\
& =\int\left(-\nabla p_{t}+\rho(f-v \cdot \nabla v)_{t}+\rho_{t}\left(f-v \cdot \nabla v-u_{t}\right)\right) \cdot u_{t t} d x
\end{aligned}
$$


We can estimate the first two terms in the right hand side of (3.13) as follows:

$$
\begin{aligned}
-\int \nabla p_{t} \cdot u_{t t} d x=\int p_{t} \operatorname{div} u_{t t} d x & =\frac{d}{d t} \int p_{t} \operatorname{div} u_{t} d x-\int p_{t t} \operatorname{div} u_{t} d x \\
& \leq \frac{d}{d t} \int p_{t} \operatorname{div} u_{t} d x+\left|p_{t t}\right|_{L^{2}}^{2}+\left|\nabla u_{t}\right|_{L^{2}}^{2}
\end{aligned}
$$

and

$$
\begin{aligned}
\int \rho(f-v \cdot \nabla v)_{t} \cdot u_{t t} d x & \leq C|\rho|_{L^{\infty}}^{\frac{1}{2}}\left(\left|f_{t}\right|_{L^{2}}+|v|_{D_{0}^{1} \cap D^{2}}\left|v_{t}\right|_{D_{0}^{1}}\right)\left|\sqrt{\rho} u_{t t}\right|_{L^{2}} \\
& \leq C c_{0}\left(\left|f_{t}\right|_{L^{2}}^{2}+c_{3}^{2}\left|v_{t}\right|_{D_{0}^{1}}^{2}\right)+\frac{1}{2}\left|\sqrt{\rho} u_{t t}\right|_{L^{2}}^{2} .
\end{aligned}
$$

To estimate the last term, we observe that

$$
\begin{aligned}
\int \rho_{t}(f-v \cdot \nabla v) \cdot u_{t t} d x \\
=\frac{d}{d t} \int \rho_{t}(f-v \cdot \nabla v) \cdot u_{t} d x-\int \rho_{t t}(f-v \cdot \nabla v) \cdot u_{t} d x \\
\quad-\int \rho_{t}(f-v \cdot \nabla v)_{t} \cdot u_{t} d x
\end{aligned}
$$

and

$$
-\int \rho_{t} u_{t} \cdot u_{t t} d x=-\frac{d}{d t} \int \rho_{t}\left(\frac{1}{2}\left|u_{t}\right|^{2}\right) d x+\int \rho_{t t}\left(\frac{1}{2}\left|u_{t}\right|^{2}\right) d x .
$$

Then by virtue of Lemma 3.2, we obtain

$$
\begin{aligned}
&-\int \rho_{t t}(f-v \cdot \nabla v) \cdot u_{t} d x \leq C\left|\rho_{t t}\right|_{L^{2}}\left(|f|_{H^{1}}+|v|_{D_{0}^{1} \cap D^{2}}^{2}\right)\left|\nabla u_{t}\right|_{L^{2}} \\
& \leq C c_{3}^{4}\left|\rho_{t t}\right|_{L^{2}}^{2}+\left|\nabla u_{t}\right|_{L^{2}}^{2} \\
&-\int \rho_{t}(f-v \cdot \nabla v)_{t} \cdot u_{t} d x \leq C\left|\rho_{t}\right|_{L^{3}}\left(\left|f_{t}\right|_{L^{2}}+|v|_{D_{0}^{1} \cap D^{2}}\left|v_{t}\right|_{D_{0}^{1}}\right)\left|\nabla u_{t}\right|_{L^{2}} \\
& \leq C c_{3}^{4}\left(\left|f_{t}\right|_{L^{2}}^{2}+c_{3}^{2}\left|v_{t}\right|_{D_{0}^{1}}^{2}\right)+\left|\nabla u_{t}\right|_{L^{2}}^{2}
\end{aligned}
$$

and

$$
\begin{aligned}
\int \rho_{t t}\left(\frac{1}{2}\left|u_{t}\right|^{2}\right) d x & =-\int \operatorname{div}\left(\rho_{t} v+\rho v_{t}\right)\left(\frac{1}{2}\left|u_{t}\right|^{2}\right) d x \\
& \leq \int\left(\left|\rho_{t}\right||v|+\rho\left|v_{t}\right|\right)\left|u_{t}\right|\left|\nabla u_{t}\right| d x \\
& \leq C c_{3}^{3}\left|\nabla u_{t}\right|_{L^{2}}^{2}+C c_{0}^{\frac{3}{4}}\left|v_{t}\right|_{D_{0}^{1}}\left|\sqrt{\rho} u_{t}\right|_{L^{2}}^{\frac{1}{2}}\left|\nabla u_{t}\right|_{L^{2}}^{\frac{3}{2}} \\
& \leq C c_{3}^{3}\left|\nabla u_{t}\right|_{L^{2}}^{2}+\left(1+c_{3}\right)^{-1}\left|v_{t}\right|_{D_{0}^{1}}^{2}\left|\sqrt{\rho} u_{t}\right|_{L^{2}}\left|\nabla u_{t}\right|_{L^{2}} \\
& \leq C c_{3}^{3}\left|u_{t}\right|_{D_{0}^{1}}^{2}+\left(1+c_{3}\right)^{-1}\left|v_{t}\right|_{D_{0}^{1}}^{2}\left(\left|\sqrt{\rho} u_{t}\right|_{L^{2}}^{2}+\left|\nabla u_{t}\right|_{L^{2}}^{2}\right) .
\end{aligned}
$$


Substituting all the above estimates into (3.13), we have

$$
\begin{gathered}
\int \rho\left|u_{t t}\right|^{2} d x+\frac{d}{d t} \int \mu\left|\nabla u_{t}\right|^{2}+(\lambda+\mu)\left(\operatorname{div} u_{t}\right)^{2} d x \\
\leq \frac{d}{d t} \int\left(2 p_{t} \operatorname{div} u_{t}+2 \rho_{t}(f-v \cdot \nabla v) \cdot u_{t}-\rho_{t}\left|u_{t}\right|^{2}\right) d x \\
\quad+C\left(\left|p_{t t}\right|_{L^{2}}^{2}+c_{3}^{4}\left|\rho_{t t}\right|_{L^{2}}^{2}+c_{3}^{4}\left|f_{t}\right|_{L^{2}}^{2}+c_{3}^{6}\left|v_{t}\right|_{D_{0}^{1}}^{2}+c_{3}^{3}\left|u_{t}\right|_{D_{0}^{1}}^{2}\right) \\
\quad+\left|v_{t}\right|_{D_{0}^{1}}^{2}\left|\sqrt{\rho} u_{t}\right|_{L^{2}}^{2}+\left(1+c_{3}\right)^{-1}\left|v_{t}\right|_{D_{0}^{1}}^{2}\left|\nabla u_{t}\right|_{L^{2}}^{2}
\end{gathered}
$$

for $0 \leq t \leq \min \left(T_{*}, T_{3}\right)$. Now let us define a function $\Lambda$ by

$$
\begin{aligned}
\Lambda(t)=\int & \left(\mu\left|\nabla u_{t}\right|^{2}+(\lambda+\mu)\left(\operatorname{div} u_{t}\right)^{2}\right)(t) d x \\
& -\int\left(2 p_{t} \operatorname{div} u_{t}+2 \rho_{t}(f-v \cdot \nabla v) \cdot u_{t}-\rho_{t}\left|u_{t}\right|^{2}\right)(t) d x
\end{aligned}
$$

Then it follows from Lemma 3.2, Lemma 3.4 and (3.12) that

$$
\begin{aligned}
& |\Lambda| \leq C\left(\left|\nabla u_{t}\right|_{L^{2}}^{2}+\left|p_{t}\right|_{L^{2}}^{2}+\left|\rho_{t}\right|_{L^{3}}^{2}|f-v \cdot \nabla v|_{L^{2}}^{2}+|\rho|_{L^{\infty}}^{3}|v|_{D_{0}^{1}}^{4}\left|\sqrt{\rho} u_{t}\right|_{L^{2}}^{2}\right) \\
& \quad \leq C\left|\nabla u_{t}\right|_{L^{2}}^{2}+M\left(c_{1}\right) c_{3}^{8}, \\
& \quad \Lambda \geq C^{-1}\left|\nabla u_{t}\right|_{L^{2}}^{2}-M\left(c_{1}\right) c_{3}^{8} \text { and }|\Lambda(0)| \leq M\left(c_{1}\right) c_{3}^{8} .
\end{aligned}
$$

Hence integrating (3.14) over $(0, t)$ and using Lemma 3.2 and Lemma 3.4, we deduce that

$$
\begin{aligned}
& \int_{0}^{t}\left|\sqrt{\rho} u_{t t}(s)\right|_{L^{2}}^{2} d s+\left|\nabla u_{t}(t)\right|_{L^{2}}^{2} \\
& \quad \leq M\left(c_{1}\right) c_{3}^{12}+\int_{0}^{t} C\left(1+c_{3}\right)^{-1}\left|v_{t}\right|_{D_{0}^{1}}^{2}\left|\nabla u_{t}(s)\right|_{L^{2}}^{2} d s
\end{aligned}
$$

for $0 \leq t \leq \min \left(T_{*}, T_{3}\right)$. Therefore, in view of Gronwall's inequality, we conclude that

$$
\int_{0}^{t}\left|\sqrt{\rho} u_{t t}(s)\right|_{L^{2}}^{2} d s+\left|u_{t}(t)\right|_{D_{0}^{1}}^{2} \leq M\left(c_{1}\right) c_{3}^{12}
$$

for $0 \leq t \leq \min \left(T_{*}, T_{3}\right)$. Moreover, since $L u=-\nabla p+\rho\left(f-v \cdot \nabla v-u_{t}\right)$ in $\Omega$, it follows from the elliptic regularity result that

$$
\int_{0}^{t}\left|u_{t}(s)\right|_{D^{2}}^{2} d s+|u(t)|_{D^{3}}^{2} \leq M\left(c_{1}\right) c_{3}^{12} \quad \text { for } \quad 0 \leq t \leq \min \left(T_{*}, T_{3}\right) .
$$

This completes the proof of Lemma 3.5.

From Lemma 3.2-Lemma 3.5, it follows that

$$
\begin{array}{r}
|u(t)|_{D_{0}^{1}}+\int_{0}^{t}|u(s)|_{D^{2}}^{2} d s \leq M\left(c_{1}\right), \\
|u(t)|_{D^{2}}+\int_{0}^{t}\left(\left|u_{t}(s)\right|_{D_{0}^{1}}^{2}+|u(s)|_{D^{3}}^{2}\right) d s \leq M\left(c_{1}\right) c_{2}^{\frac{3}{2}} c_{3}^{\frac{1}{2}}, \\
\left|u_{t}(t)\right|_{D_{0}^{1}}+|u(t)|_{D^{3}}+\int_{0}^{t}\left(\left|u_{t}(s)\right|_{D^{2}}^{2}+|u(s)|_{D^{4}}^{2}\right) d s \leq M\left(c_{1}\right) c_{3}^{12}, \\
\left|\rho(t)-\rho^{\infty}\right|_{H^{3}}+\left|\rho_{t}(t)\right|_{H^{2}}+\left|\sqrt{\rho} u_{t}(t)\right|_{L^{2}} \leq M\left(c_{1}\right) c_{3}^{12}
\end{array}
$$


for $0 \leq t \leq \min \left(T_{*}, T_{3}\right)$. Here $M=M(\cdot)$ is a fixed increasing continuous function on $[1, \infty)$ which depends only on the parameters of $C$. Therefore, defining the constants $c_{i}$ 's and $T_{*}$ by

$$
c_{1}=M\left(c_{0}\right), \quad c_{2}=M\left(c_{1}\right), \quad c_{3}=c_{2}^{5}, \quad c_{4}=c_{2} c_{3}^{12}
$$

and

$$
T_{*}=\min \left(T, T_{3}\right) \quad \text { with } \quad T_{3}=\left(1+c_{4}\right)^{-9},
$$

we conclude that

$$
\begin{array}{r}
\sup _{0 \leq t \leq T_{*}}|u(t)|_{D_{0}^{1}}+\int_{0}^{T_{*}}|u(t)|_{D^{2}}^{2} d t \leq c_{2}, \\
\sup _{0 \leq t \leq T_{*}}|u(t)|_{D^{2}}+\int_{0}^{T_{*}}\left(\left|u_{t}(t)\right|_{D_{0}^{1}}^{2}+|u(t)|_{D^{3}}^{2}\right) d t \leq c_{3}, \\
\operatorname{ess} \sup _{0 \leq t \leq T_{*}}\left(\left|u_{t}(t)\right|_{D_{0}^{1}}+|u(t)|_{D^{3}}\right)+\int_{0}^{T_{*}}\left(\left|u_{t}(t)\right|_{D^{2}}^{2}+|u(t)|_{D^{4}}^{2}\right) d t \leq c_{4}, \\
\operatorname{ess}_{0 \leq t \leq T_{*}}\left(\left|\rho(t)-\rho^{\infty}\right|_{H^{3}}+\left|\rho_{t}(t)\right|_{H^{2}}+\left|\sqrt{\rho} u_{t}(t)\right|_{L^{2}}\right) \leq c_{4} .
\end{array}
$$

\section{Proof of Theorem 1.1}

Let $\left(\rho_{0}, u_{0}, f\right)$ be a given data satisfying the hypotheses of Theorem 1.1. To prove the existence, we construct a sequence $\left\{\left(\rho^{k}, u^{k}\right)\right\}_{k>1}$ of approximate solutions solving the linearized problem (3.1)-(3.4) successively. First, let $F \in$ $C\left([0, \infty) ; H^{1}\right) \cap L^{2}\left(0, \infty ; H^{2}\right)$ be the solution of the heat equation $F_{t}-\Delta F=0$ in $(0, \infty) \times \Omega$ with $F(0)=-\nabla p\left(\rho_{0}\right)+\rho_{0}\left(f(0)+g_{2}\right) \in H^{1}$. Then since $u_{0} \in D_{0}^{1} \cap D^{3}$ and $F(0)-L u_{0}=0 \in D_{0}^{1}$, we can easily show that there exists a unique solution $w=u^{0} \in C\left([0, \infty) ; D_{0}^{1} \cap D^{3}\right) \cap L^{2}\left(0, \infty ; D^{4}\right)$ to the following linear parabolic problem

$$
w_{t}+L w=F \quad \text { in } \quad(0, \infty) \times \Omega \quad \text { and } \quad w(0)=u_{0} \quad \text { in } \quad \Omega .
$$

It is also easy to show that

$$
\begin{aligned}
\sup _{0 \leq t \leq 1}\left(\left|u^{0}(t)\right|_{D_{0}^{1} \cap D^{3}}+\left|u_{t}^{0}(t)\right|_{D_{0}^{1}}\right) & +\int_{0}^{1}\left(\left|u_{t}^{0}(t)\right|_{D^{2}}^{2}+\left|u^{0}(t)\right|_{D^{4}}^{2}\right) d t \\
\leq & C\left(1+|F(0)|_{H^{1}}^{2}+\left|u_{0}\right|_{D_{0}^{1} \cap D^{3}}^{2}\right) .
\end{aligned}
$$

Let us define $c_{0}$ by

$$
c_{0}=2+\rho^{\infty}+\left|\rho_{0}-\rho^{\infty}\right|_{H^{3}}+\left|u_{0}\right|_{D_{0}^{1}}+\left|\sqrt{\rho_{0}} g_{2}\right|_{L^{2}}+\left|g_{2}\right|_{D_{0}^{1}},
$$

and we choose the positive constants $c_{1}, c_{2}, c_{3}, c_{4}$ and $T_{*}$ as in (3.15) and (3.16), which are dependent only on $c_{0}$ and the parameters of $C$. Then since $u_{0} \in D_{0}^{1} \cap D^{3}$ is a solution to the elliptic system

$$
L u_{0}=F(0)=-\nabla p\left(\rho_{0}\right)+\rho_{0}\left(f(0)+g_{2}\right) \quad \text { in } \quad \Omega
$$

and

$$
|F(0)|_{H^{1}}=\left|-\nabla p\left(\rho_{0}\right)+\rho_{0}\left(f(0)+g_{2}\right)\right|_{H^{1}} \leq M\left(c_{0}\right),
$$


it follows from the elliptic regularity result in [3] that

$$
\left|u_{0}\right|_{D_{0}^{1} \cap D^{3}} \leq C\left(|F(0)|_{H^{1}}+\left|u_{0}\right|_{D_{0}^{1}}\right) \leq M\left(c_{0}\right) .
$$

By virtue of (3.15), (4.1), (4.2) and (4.3), we may assume without loss of generality that

$$
\sup _{0 \leq t \leq T_{*}}\left(\left|u^{0}(t)\right|_{D_{0}^{1} \cap D^{3}}+\left|u_{t}^{0}(t)\right|_{D_{0}^{1}}\right)+\int_{0}^{T_{*}}\left(\left|u_{t}^{0}(t)\right|_{D^{2}}^{2}+\left|u^{0}(t)\right|_{D^{4}}^{2}\right) d t \leq c_{1} .
$$

The construction of the sequence $\left\{\left(\rho^{k}, u^{k}\right)\right\}_{k \geq 1}$ is based on the following key lemma to the proof of Theorem 1.1.

Lemma 4.1. Let $v$ be a vector field satisfying the regularity (3.5) with $T$ replaced by $T_{*}$. Assume further that $v$ satisfies the following estimate

$$
\begin{array}{r}
|v(0)|_{D_{0}^{1} \cap D^{3}} \leq c_{1}, \\
\sup _{0 \leq t \leq T_{*}}|v(t)|_{D_{0}^{1}}+\int_{0}^{T_{*}}|v(t)|_{D^{2}}^{2} d t \leq c_{2}, \\
\sup _{0 \leq t \leq T_{*}}|v(t)|_{D^{2}}+\int_{0}^{T_{*}}\left(\left|v_{t}(t)\right|_{D_{0}^{1}}^{2}+|v(t)|_{D^{3}}^{2}\right) d t \leq c_{3}, \\
\operatorname{esssup}_{0 \leq t \leq T_{*}}\left(\left|v_{t}(t)\right|_{D_{0}^{1}}+|v(t)|_{D^{3}}\right)+\int_{0}^{T_{*}}\left(\left|v_{t}(t)\right|_{D^{2}}^{2}+|v(t)|_{D^{4}}^{2}\right) d t \leq c_{4} .
\end{array}
$$

Then there exists a unique solution $(\rho, u)$ to the linearized problem (3.1)-(3.4) satisfying the estimate (3.17) as well as the regularity

$$
\begin{gathered}
\rho-\rho^{\infty} \in C\left(\left[0, T_{*}\right] ; H^{3}\right), \quad u \in C\left(\left[0, T_{*}\right] ; D_{0}^{1} \cap D^{3}\right) \cap L^{2}\left(0, T_{*} ; D^{4}\right), \\
u_{t} \in L^{\infty}\left(0, T_{*} ; D_{0}^{1}\right) \cap L^{2}\left(0, T_{*} ; D^{2}\right) \quad \text { and } \quad \sqrt{\rho} u_{t} \in L^{\infty}\left(0, T_{*} ; L^{2}\right) .
\end{gathered}
$$

Proof. Let $R_{0}>1$ be a sufficiently large number so that

$$
\Omega \subset B_{R_{0} / 2} \text { if } \Omega \subset \subset \mathbf{R}^{3} ; \quad \mathbf{R}^{3} \backslash \Omega \subset B_{R_{0} / 2} \quad \text { if } \quad \mathbf{R}^{3} \backslash \Omega \subset \subset \mathbf{R}^{3},
$$

and we define

$$
\begin{gathered}
\varphi^{R}(x)=\varphi(x / R), \quad g_{2}^{R}(x)=\varphi^{R}(x) g_{2}(x), \\
v^{R}(t, x)=\varphi^{R}(x) v(t, x) \quad \text { and } \quad f^{R}(t, x)=\varphi^{R}(x) f(t, x)
\end{gathered}
$$

for $(t, x) \in\left[0, T_{*}\right] \times \Omega$, where $\varphi \in C_{c}^{\infty}\left(B_{1}\right)$ is a smooth cut-off function such that $\varphi=1$ in $B_{1 / 2}$. Note that if $\Omega \subset \subset \mathbf{R}^{3}$, then $g_{2}^{R}=g_{2}, v^{R}=v$ and $f^{R}=f$ for each $R>R_{0}$ and otherwise, they are supported in $\Omega_{R}$ or $\left[0, T_{*}\right] \times \Omega_{R}$, where $\Omega_{R}=\Omega \cap B_{R}$ 1 .

For each $R>R_{0}$, let $u_{0}^{R} \in H_{0}^{1}\left(\Omega_{R}\right) \cap H^{3}\left(\Omega_{R}\right)$ be a unique solution to the elliptic boundary value problem

$$
L u_{0}^{R}=F_{0}^{R} \quad \text { in } \quad \Omega_{R} \quad \text { and } \quad u_{0}^{R}=0 \quad \text { on } \quad \partial \Omega_{R},
$$

\footnotetext{
${ }^{1}$ If $\Omega$ is the half space $\mathbf{R}^{2} \times \mathbf{R}_{+}$, then the non-smooth domain $\Omega_{R}$ should be replaced by a smooth domain $\tilde{\Omega}_{R}$ such that $\Omega_{R} \subset \tilde{\Omega}_{R} \subset \Omega_{2 R}$.
} 
where

$$
F_{0}^{R}=-\nabla p\left(\rho_{0}^{R}\right)+\rho_{0}^{R}\left(f^{R}(0)+g_{2}^{R}\right) \quad \text { and } \quad \rho_{0}^{R}=\rho_{0}+R^{-3} .
$$

Then we extend $u_{0}^{R}$ to $\Omega$ by defining zero outside $\Omega_{R}$. We will show that

$$
u_{0}^{R} \rightarrow u_{0} \quad \text { in } \quad D_{0}^{1}(\Omega) \quad \text { as } \quad R \rightarrow \infty .
$$

To do this, we first observe that

$$
L u_{0}=-\nabla p\left(\rho_{0}\right)+\rho_{0}\left(f(0)+g_{2}\right) \equiv F_{0} \quad \text { in } \quad \Omega .
$$

From (4.7) and (4.9), it follows that $L\left(u_{0}^{R}-u_{0}\right)=F_{0}^{R}-F_{0}$ in $\Omega_{R}$. Hence noting that $u_{0}^{R} \in H_{0}^{1}\left(\Omega_{R}\right)$, we obtain

$$
\begin{aligned}
& \int_{\Omega_{R}} \mu\left|\nabla u_{0}^{R}\right|^{2}+(\lambda+\mu)\left(\operatorname{div} u_{0}^{R}\right)^{2} d x \\
& =\int_{\Omega_{R}} \mu \nabla u_{0}: \nabla u_{0}^{R}+(\lambda+\mu) \operatorname{div} u_{0} \operatorname{div} u_{0}^{R} d x+\int_{\Omega_{R}}\left(F_{0}^{R}-F_{0}\right) \cdot u_{0}^{R} d x .
\end{aligned}
$$

The second term of the right hand side in (4.10) is bounded by

$$
\begin{aligned}
\int_{\Omega_{R}}\left(F_{0}^{R}-F_{0}\right) \cdot u_{0}^{R} d x \leq & \int_{\Omega_{R}}\left|p\left(\rho_{0}^{R}\right)-p\left(\rho_{0}\right)\right|\left|\nabla u_{0}^{R}\right| d x \\
& +R^{-3} \int_{\Omega_{R}}\left(|f(0)|+\left|g_{2}\right|\right)\left|u_{0}^{R}\right| d x \\
& +\int_{\Omega_{R}} \rho_{0}\left(\varphi^{R}-1\right)\left(f(0)+g_{2}\right) \cdot u_{0}^{R} d x
\end{aligned}
$$

while

$$
\begin{gathered}
\int_{\Omega_{R}}\left|p\left(\rho_{0}^{R}\right)-p\left(\rho_{0}\right)\right|\left|\nabla u_{0}^{R}\right| d x \leq R^{-\frac{3}{2}} M\left(c_{0}\right)\left|\nabla u_{0}^{R}\right|_{L^{2}} \\
R^{-3} \int_{\Omega_{R}}\left(|f(0)|+\left|g_{2}\right|\right)\left|u_{0}^{R}\right| d x \leq C R^{-1}\left(|f(0)|_{H^{1}}+\left|g_{2}\right|_{D_{0}^{1}}\right)\left|\nabla u_{0}^{R}\right|_{L^{2}}
\end{gathered}
$$

and

$$
\begin{aligned}
& \int_{\Omega_{R}} \rho_{0}\left(\varphi^{R}-1\right)\left(f(0)+g_{2}\right) \cdot u_{0}^{R} d x \\
& \quad=\int_{\Omega_{R}}\left(\varphi^{R}-1\right)\left(L u_{0}+\nabla p\left(\rho_{0}\right)\right) \cdot u_{0}^{R} d x \\
& \quad \leq C \int_{\Omega_{R}}\left(\left|\nabla \varphi^{R}\right|\left|u_{0}^{R}\right|+\left|\varphi^{R}-1\right|\left|\nabla u_{0}^{R}\right|\right)\left(\left|\nabla u_{0}\right|+\left|p\left(\rho_{0}\right)-p\left(\rho^{\infty}\right)\right|\right) d x \\
& \quad \leq M\left(c_{0}\right)\left(\left|\nabla u_{0}\right|_{L^{2}\left(\Omega \backslash \Omega_{R / 2}\right)}+\left|\rho_{0}-\rho^{\infty}\right|_{L^{2}\left(\Omega \backslash \Omega_{R / 2}\right)}\right)\left|\nabla u_{0}^{R}\right|_{L^{2}} .
\end{aligned}
$$

Hence from (4.10), it follows that

$$
\left|u_{0}^{R}\right|_{D_{0}^{1}(\Omega)} \leq C\left|u_{0}\right|_{D_{0}^{1}(\Omega)}+o(1) \quad \text { and } \quad \int_{\Omega}\left(F_{0}^{R}-F_{0}\right) \cdot u_{0}^{R} d x=o(1)
$$

where $o(1)$ denotes a function of $R$ which tends to zero as $R \rightarrow \infty$. This means that there exists a sequence $\left\{R_{j}\right\}, R_{j} \rightarrow \infty$, such that $\left\{u_{0}^{R_{j}}\right\}$ converges weakly in $D_{0}^{1}(\Omega)$ to a limit $u_{0}^{\infty}$. It is easy to show that $L u_{0}^{\infty}=L u_{0}$ in $D^{-1}(\Omega)$, where $D^{-1}(\Omega)$ denotes the dual space of $D_{0}^{1}(\Omega)$. Hence it follows that $u_{0}^{\infty}=u_{0}$ in $\Omega$ and $\left\{u_{0}^{R_{j}}\right\}$ converges weakly in $D_{0}^{1}(\Omega)$ to $u_{0}$. Then by virtue of (4.10) and (4.11), we 
deduce that $\left\{u_{0}^{R_{j}}\right\}$ converges strongly to $u_{0}$ in $D_{0}^{1}(\Omega)$. Since the above argument also shows that every subsequence of $\left\{u_{0}^{R}\right\}$ has a subsequence converging in $D_{0}^{1}(\Omega)$ to the same limit $u_{0}$, we conclude that the whole sequence $\left\{u_{0}^{R}\right\}$ converges to $u_{0}$ in $D_{0}^{1}(\Omega)$ as $R \rightarrow \infty$, which proves (4.8).

We are now ready to prove Lemma 4.1. To prove the existence, we consider the following initial boundary value problem

$$
\begin{array}{rrr}
\rho_{t}+\operatorname{div}\left(\rho v^{R}\right)=0 & \text { in }\left(0, T_{*}\right) \times \Omega_{R}, \\
\rho u_{t}+L u+\nabla p(\rho)=\rho\left(f^{R}-v^{R} \cdot \nabla v^{R}\right) & \text { in }\left(0, T_{*}\right) \times \Omega_{R}, \\
\left.(\rho, u)\right|_{t=0}=\left(\rho_{0}^{R}, u_{0}^{R}\right) \quad \text { in } \quad \Omega_{R} \quad \text { and } u=0 \text { on }\left(0, T_{*}\right) \times \partial \Omega_{R} .
\end{array}
$$

Since $\rho_{0}^{R} \geq R^{-3}>0$ in $\Omega_{R}$, it follows from Lemma 3.1 that for each $R>R_{0}$, there exists a unique strong solution $(\rho, u)=\left(\rho^{R}, u^{R}\right)$ to the problem (4.12), (4.13) and (4.14). It is easy to show that

$$
\begin{aligned}
& \left|v^{R}-v\right|_{C\left(\left[0, T_{*}\right] ; D_{0}^{1} \cap D^{3}\right)}+\left|\left(v^{R}\right)_{t}-v_{t}\right|_{L^{\infty}\left(0, T_{*} ; D_{0}^{1}\right) \cap L^{2}\left(0, T_{*} ; D^{2}\right)} \rightarrow 0 \\
& \text { and }\left|\sqrt{\rho}_{0}^{R} g_{2}^{R}-\sqrt{\rho_{0}} g_{2}\right|_{L^{2}}+\left|g_{2}^{R}-g_{2}\right|_{D_{0}^{1}} \rightarrow 0 \quad \text { as } \quad R \rightarrow \infty .
\end{aligned}
$$

Combining this, (4.5) and (4.8), we deduce that there exists a large number $R_{1}>R_{0}$ such that for all $R>R_{1}, v_{R}$ satisfies the estimate (3.7) with the spatial domain being $\Omega_{R}$ and

$$
\begin{aligned}
& 1+\left(\rho^{\infty}+R^{-3}\right)+\left|\rho_{0}^{R}-\left(\rho^{\infty}+R^{-3}\right)\right|_{H^{3}\left(\Omega_{R}\right)} \\
& \quad+\left|u_{0}^{R}\right|_{D_{0}^{1}\left(\Omega_{R}\right)}+\left|\sqrt{\rho_{0}^{R}} g_{2}^{R}\right|_{L^{2}\left(\Omega_{R}\right)}+\left|g_{2}^{R}\right|_{D_{0}^{1}\left(\Omega_{R}\right)}<c_{0} .
\end{aligned}
$$

Therefore, from the results in Section 3, we conclude that for each $R>R_{1}$, the solution $\left(\rho^{R}, u^{R}\right)$ satisfies the estimate (3.17) with the domain being $\Omega_{R}$. We extend $\left(\rho^{R}, u^{R}\right)$ by defining zero outside $\Omega_{R}$. Then by virtue of the uniform estimate $(3.17)$ on $R$, we deduce that there exists a sequence $\left\{R_{j}\right\}, R_{j} \rightarrow \infty$, such that $\left\{\left(\rho^{R_{j}}, u^{R_{j}}\right)\right\}$ converges in a weak or weak-* sense to a limit $(\rho, u)$. Moreover, since $(\rho, u)$ also satisfies (3.17) with the domain being $\Omega_{R}$ for each $R>R_{1}$, it follows that

$$
\begin{gathered}
\rho-\rho^{\infty} \in L^{\infty}\left(0, T_{*} ; H^{3}\right), \quad u \in L^{\infty}\left(0, T_{*} ; D_{0}^{1} \cap D^{3}\right) \cap L^{2}\left(0, T_{*} ; D^{4}\right), \\
u_{t} \in L^{\infty}\left(0, T_{*} ; D_{0}^{1}\right) \cap L^{2}\left(0, T_{*} ; D^{2}\right) \quad \text { and } \sqrt{\rho} u_{t} \in L^{\infty}\left(0, T_{*} ; L^{2}\right) .
\end{gathered}
$$

We will show that $(\rho, u)$ is a solution to the original problem (3.1)-(3.4). It is obvious that $(\rho, u)$ satisfies the boundary conditions in (3.3) and (3.4). Let $R>R_{1}$ be a fixed large number. Then since for all sufficiently large $j,\left(\rho^{R_{j}}, u^{R_{j}}\right)$ satisfies the uniform estimate (3.17) with the domain being $\Omega_{R}$, it follows from a standard compactness result (see [22] for instance) that a subsequence of $\left\{\left(\rho^{R_{j}}, u^{R_{j}}\right)\right\}$ converges to $(\rho, u)$ in $C\left(\left[0, T_{*}\right] ; H^{1}\left(\Omega_{R}\right)\right)$. Using this result together with (4.8), we can show that $(\rho, u)$ satisfies the equations (3.1) and $(3.2)$ in $\left(0, T_{*}\right) \times \Omega_{R}$ and $(\rho(0), u(0))=\left(\rho_{0}, u_{0}\right)$ in $\Omega_{R}$. Since $R$ can be arbitrarily large, we have proved the existence of a solution $(\rho, u)$ to the original problem (3.1)-(3.4) satisfying the regularity (4.15). The uniqueness of solutions with this regularity is easily proved. Hence it remains to prove the time-continuity of the solution $(\rho, u)$. First, from a 
classical embedding result, we deduce that $u \in C\left(\left[0, T_{*}\right] ; D_{0}^{1} \cap D^{3}\right)$. Then the timecontinuity of $\rho$ follows immediately from Lemma 2.1. This completes the proof of Lemma 4.1.

We turn to the proof of Theorem 1.1. We first observe that by virtue of (4.4), the vector field $v=u^{0}$ satisfies the hypotheses of Lemma 4.1. Hence it follows from Lemma 4.1 that there exists a unique strong solution $(\rho, u)=\left(\rho^{1}, u^{1}\right)$ to the linearized problem (3.1)-(3.4) with $v=u^{0}$, which satisfies the regularity estimate (3.17). Then an obvious inductive argument allows us to construct approximate solutions $\left(\rho^{k}, u^{k}\right)$ for all $k \geq 1$ : assuming that $u^{k-1}$ was defined for $k \geq 1$, let $\left(\rho^{k}, u^{k}\right)$ be the unique solution to the problem (3.1)-(3.4) with $v=u^{k-1}$. Then since $u^{k}(0)=u_{0}$ for each $k \geq 0$, it follows from Lemma 4.1 that there exists a constant $\tilde{C}>1$ such that

$$
\begin{array}{r}
\sup _{0 \leq t \leq T_{*}}\left(\left|\rho^{k}(t)-\rho^{\infty}\right|_{H^{3}}+\left|\rho_{t}(t)\right|_{H^{2}}+\left|u^{k}(t)\right|_{D_{0}^{1} \cap D^{3}}\right) \leq \tilde{C}, \\
\operatorname{ess\operatorname {sup}}\left(\left|u_{t}^{k}(t)\right|_{D_{0}^{1}}+\left|\sqrt{\rho}^{k} u_{t}^{k}(t)\right|_{L^{2}}\right)+\int_{0}^{T_{*}}\left(\left|u_{t}^{k}(t)\right|_{D^{2}}^{2}+|u(t)|_{D^{4}}^{2}\right) d t \leq \tilde{C}
\end{array}
$$

for all $k \geq 1$. Throughout the proof, we denote by $\tilde{C}$ a generic positive constant depending only on $c_{0}$ and the parameters of $C$, but independent of $k$.

From now on, we show that the full sequence $\left\{\left(\rho^{k}, u^{k}\right)\right\}$ of approximate solutions converges to a solution to the original problem (1.1)-(1.5) in a strong sense. To do this, let us define

$$
\bar{\rho}^{k+1}=\rho^{k+1}-\rho^{k}, \quad \bar{u}^{k+1}=u^{k+1}-u^{k} \quad \text { and } \quad p^{k}=p\left(\rho^{k}\right) .
$$

Then from the equation (3.1), we derive

$$
\bar{\rho}_{t}^{k+1}+\operatorname{div}\left(\bar{\rho}^{k+1} u^{k}\right)+\operatorname{div}\left(\rho^{k} \bar{u}^{k}\right)=0 .
$$

Multiplying this by $\bar{\rho}^{k+1}$ and integrating over $\Omega$, we obtain

$$
\begin{aligned}
& \frac{d}{d t} \int\left|\bar{\rho}^{k+1}\right|^{2} d x \\
& \quad \leq C \int\left|\nabla u^{k}\right|\left|\bar{\rho}^{k+1}\right|^{2}+\left(\left|\nabla \rho^{k}\right|\left|\bar{u}^{k}\right|+\rho^{k}\left|\nabla \bar{u}^{k}\right|\right)\left|\bar{\rho}^{k+1}\right| d x \\
& \quad \leq C\left|\nabla u^{k}\right|_{L^{\infty}}\left|\bar{\rho}^{k+1}\right|_{L^{2}}^{2}+C\left(\left|\nabla \rho^{k}\right|_{H^{1}}+\left|\rho^{k}\right|_{L^{\infty}}\right)\left|\nabla \bar{u}^{k}\right|_{L^{2}}\left|\bar{\rho}^{k+1}\right|_{L^{2}}
\end{aligned}
$$

Hence it follows from the uniform bound (4.16) that

$$
\frac{d}{d t}\left|\bar{\rho}^{k+1}\right|_{L^{2}}^{2} \leq \eta^{-1} \tilde{C}\left|\bar{\rho}^{k+1}\right|_{L^{2}}^{2}+\eta\left|\nabla \bar{u}^{k}\right|_{L^{2}}
$$

for $0 \leq t \leq T_{*}$, where $\eta \in(0,1)$ is a small number. 
In case that $\rho^{\infty}=0$, we need an estimate for $\left|\bar{\rho}^{k+1}\right|_{L^{\frac{3}{2}}}$ in addition to (4.18). Multiplying (4.17) by $\operatorname{sgn}\left(\bar{\rho}^{k+1}\right)\left|\bar{\rho}^{k+1}\right|^{\frac{1}{2}}$ and integrating over $\Omega$, we get

$$
\begin{aligned}
& \frac{d}{d t} \int\left|\bar{\rho}^{k+1}\right|^{\frac{3}{2}} d x \\
& \leq C \int\left|\nabla u^{k}\right|\left|\bar{\rho}^{k+1}\right|^{\frac{3}{2}}+\left(\left|\nabla \rho^{k}\right|\left|\bar{u}^{k}\right|+\rho^{k}\left|\nabla \bar{u}^{k}\right|\right)\left|\bar{\rho}^{k+1}\right|^{\frac{1}{2}} d x
\end{aligned}
$$

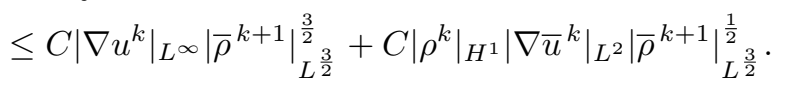

Hence multiplying this by $\left|\bar{\rho}^{k+1}\right|_{L^{\frac{3}{2}}}^{\frac{1}{2}}$ and using (4.16), we have

$$
\frac{d}{d t}\left|\bar{\rho}^{k+1}\right|_{L^{\frac{3}{2}}}^{2} \leq \eta^{-1} \tilde{C}\left|\bar{\rho}^{k+1}\right|_{L^{\frac{3}{2}}}^{2}+\eta\left|\nabla \bar{u}^{k}\right|_{L^{2}}^{2}
$$

for $0 \leq t \leq T_{*}$.

Next from the equation (3.2), we derive

$$
\begin{aligned}
\rho^{k+1} \bar{u}_{t}^{k+1}+ & \rho^{k+1} u^{k} \cdot \nabla \bar{u}^{k+1}+L \bar{u}^{k+1}+\nabla\left(p^{k+1}-p^{k}\right) \\
= & \bar{\rho}^{k+1}\left(f-u_{t}^{k}-u^{k-1} \cdot \nabla u^{k-1}\right) \\
& +\rho^{k+1}\left(u^{k} \cdot \nabla \bar{u}^{k+1}-\bar{u}^{k} \cdot \nabla u^{k}-u^{k-1} \cdot \nabla \bar{u}^{k}\right) .
\end{aligned}
$$

Multiplying this by $\bar{u}^{k+1}$, integrating over $\Omega$ and using the equation (3.1) with $(\rho, v)=\left(\rho^{k+1}, u^{k}\right)$, we obtain

$$
\begin{aligned}
& \frac{1}{2} \frac{d}{d t} \int \rho^{k+1}\left|\bar{u}^{k+1}\right|^{2} d x+\mu \int\left|\nabla \bar{u}^{k+1}\right|^{2} d x \\
& \leq C \int\left|\bar{\rho}^{k+1}\right|\left|u_{t}^{k}\right|\left|\bar{u}^{k+1}\right| d x+C \int\left|p^{k+1}-p^{k}\right|\left|\nabla \bar{u}^{k+1}\right| d x \\
& \quad+C \int\left|\bar{\rho}^{k+1}\right|\left|f-u^{k-1} \cdot \nabla u^{k-1}\right|\left|\bar{u}^{k+1}\right| d x \\
& \quad+C \int \rho^{k+1}\left(\left|u^{k}\right|\left|\nabla \bar{u}^{k+1}\right|+\left|\bar{u}^{k}\right|\left|\nabla u^{k}\right|+\left|u^{k-1}\right|\left|\nabla \bar{u}^{k}\right|\right)\left|\bar{u}^{k+1}\right| d x
\end{aligned}
$$

Using the uniform bound (4.16), we can estimate the last three integrals of the right hand side in (4.20) as follows:

$$
\begin{gathered}
C \int\left|p^{k+1}-p^{k}\right|\left|\nabla \bar{u}^{k+1}\right| d x \leq \tilde{C}\left|\bar{\rho}^{k+1}\right|_{L^{2}}^{2}+\frac{\mu}{10}\left|\nabla \bar{u}^{k+1}\right|_{L^{2}}^{2}, \\
C \int\left|\bar{\rho}^{k+1}\right|\left|f-u^{k-1} \cdot \nabla u^{k-1}\right|\left|\bar{u}^{k+1}\right| d x \\
\leq C\left|\bar{\rho}^{k+1}\right|_{L^{2}}\left|f-u^{k-1} \cdot \nabla u^{k-1}\right|_{H^{1}}\left|\nabla \bar{u}^{k+1}\right|_{L^{2}} \\
\leq \tilde{C}\left|\bar{\rho}^{k+1}\right|_{L^{2}}^{2}+\frac{\mu}{10}\left|\nabla \bar{u}^{k+1}\right|_{L^{2}}^{2}, \\
C \int \rho^{k+1}\left|u^{k}\right|\left|\nabla \bar{u}^{k+1}\right|\left|\bar{u}^{k+1}\right| d x \leq \tilde{C}\left|\sqrt{\rho^{k+1}} \bar{u}^{k+1}\right|_{L^{2}}^{2}+\frac{\mu}{10}\left|\nabla \bar{u}^{k+1}\right|_{L^{2}}^{2}
\end{gathered}
$$


and

$$
\begin{aligned}
& C \int \rho^{k+1}\left(\left|\bar{u}^{k}\right|\left|\nabla u^{k}\right|+\left|u^{k-1}\right|\left|\nabla \bar{u}^{k}\right|\right)\left|\bar{u}^{k+1}\right| d x \\
& \quad \leq C\left|\rho^{k+1}\right|_{L^{\infty}}^{\frac{1}{2}}\left(\left|u^{k}\right|_{D_{0}^{1} \cap D^{2}}+\left|u^{k-1}\right|_{D_{0}^{1} \cap D^{2}}\right)\left|\sqrt{\rho^{k+1}} \bar{u}^{k+1}\right|_{L^{2}}\left|\nabla \bar{u}^{k}\right|_{L^{2}} \\
& \quad \leq \eta^{-1} \tilde{C}\left|\sqrt{\rho^{k+1}} \bar{u}^{k+1}\right|_{L^{2}}^{2}+\eta\left|\nabla \bar{u}^{k}\right|_{L^{2}}^{2} .
\end{aligned}
$$

For the case that $\rho^{\infty}=0$ or $\Omega \subset \subset \mathbf{R}^{3}$, the first integral is readily bounded by

$$
C\left|\bar{\rho}^{k+1}\right|_{L^{\frac{3}{2}}}\left|u_{t}^{k}\right|_{D_{0}^{1}}\left|\nabla \bar{u}^{k+1}\right|_{L^{2}} \leq \tilde{C}\left|\bar{\rho}^{k+1}\right|_{L^{\frac{3}{2}}}^{2}+\frac{\mu}{10}\left|\nabla \bar{u}^{k+1}\right|_{L^{2}}^{2}
$$

For the remaining case, we assume that $\Omega$ is an unbounded domain and $\rho^{\infty}>0$. Then since $\rho_{0}-\rho^{\infty} \in H^{2}$ and $H^{2} \hookrightarrow C_{0}$, where $C_{0}$ is the space of all continuous functions on $\bar{\Omega}$ vanishing at infinity, we can choose a sufficiently large number $R>1$ (of course, independent of $k$ ) so that

$$
\frac{3}{4} \rho^{\infty} \leq \rho_{0}(x) \leq \frac{5}{4} \rho^{\infty} \quad \text { for } \quad x \in \Omega \backslash B_{R / 2} .
$$

On the other hand, it follows from Lemma 2.1 that

$$
\begin{aligned}
& \rho^{k+1}(t, x) \\
& =\rho_{0}\left(U^{k+1}(0, t, x)\right) \exp \left[-\int_{0}^{t} \operatorname{div} u^{k}\left(s, U^{k+1}(s, t, x)\right) d s\right],
\end{aligned}
$$

where $U^{k+1}=U^{k+1}(t, s, x)$ is the solution to the initial value problem

$$
\left\{\begin{aligned}
\frac{\partial}{\partial t} U^{k+1}(t, s, x) & =u^{k}\left(t, U^{k+1}(t, s, x)\right), \quad 0 \leq t \leq T_{*}, \\
U^{k+1}(s, s, x) & =x, \quad 0 \leq s \leq T_{*}, \quad x \in \bar{\Omega} .
\end{aligned}\right.
$$

In view of (4.16), we deduce that

$$
\int_{0}^{t}\left|\operatorname{div} u^{k}\left(s, U^{k+1}(s, t, x)\right)\right| d s \leq \int_{0}^{t}\left|\nabla u^{k}\right|_{L^{\infty}} d s \leq \tilde{C} t \leq \ln 2
$$

and

$$
\begin{aligned}
\left|U^{k+1}(0, t, x)-x\right| & =\left|U^{k+1}(0, t, x)-U^{k+1}(t, t, x)\right| \\
& \leq \int_{0}^{t}\left|u^{k}\left(\tau, U^{k+1}(\tau, t, x)\right)\right| d \tau \leq \tilde{C} t \leq \frac{R}{2}
\end{aligned}
$$

for all $(t, x)$ in $\left[0, T_{1}\right] \times \Omega$, where $T_{1}$ is a small positive time in $\left(0, T_{*}\right)$ which depends only on $T_{*}, R$ and the parameters of $\tilde{C}$. In particular, note that if $0 \leq t \leq T_{1}$ and $x \in \Omega \backslash B_{R}$, then $U^{k+1}(0, t, x) \in \Omega \backslash B_{R / 2}$. Hence it follows immediately from (4.21) and (4.22) that

$$
\frac{3}{8} \rho^{\infty} \leq \rho^{k+1}(t, x) \leq \frac{5}{2} \rho^{\infty} \quad \text { for } \quad(t, x) \in\left[0, T_{1}\right] \times\left(\Omega \backslash B_{R}\right) .
$$

Using this result, we can estimate the first integral in the right hand of (4.20) as follows:

$$
\begin{aligned}
C \int_{\Omega \cap B_{R}}\left|\bar{\rho}^{k+1}\right|\left|u_{t}^{k}\right|\left|\bar{u}^{k+1}\right| d x & \leq C\left|\bar{\rho}^{k+1}\right|_{L^{2}}\left|u_{t}^{k}\right|_{D_{0}^{1}}\left|\nabla \bar{u}^{k+1}\right|_{L^{2}} \\
& \leq \tilde{C}\left|\bar{\rho}^{k+1}\right|_{L^{2}}^{2}+\frac{\mu}{10}\left|\nabla \bar{u}^{k+1}\right|_{L^{2}}^{2}
\end{aligned}
$$


and

$$
\begin{aligned}
C \int_{\Omega \backslash B_{R}}\left|\bar{\rho}^{k+1}\right|\left|u_{t}^{k}\right|\left|\bar{u}^{k+1}\right| d & \leq \frac{C}{\sqrt{\rho}} \int\left|\bar{\rho}^{k+1}\right|\left|\sqrt{\rho}^{k} u_{t}^{k}\right|\left|\bar{u}^{k+1}\right| d x \\
\leq & \tilde{C}\left|\bar{\rho}^{k+1}\right|_{L^{2}}^{2}+\frac{\mu}{10}\left|\nabla \bar{u}^{k+1}\right|_{L^{2}}^{2} .
\end{aligned}
$$

Therefore, substituting all the estimates into (4.20), we deduce that

$$
\begin{aligned}
\frac{d}{d t}\left|\sqrt{\rho^{k+1}} \bar{u}^{k+1}(t)\right|_{L^{2}}^{2} & +\mu\left|\nabla \bar{u}^{k+1}(t)\right|_{L^{2}}^{2} \\
& \leq \eta^{-1} \tilde{C} \varphi^{k+1}(t)+2 \eta\left|\nabla \bar{u}^{k}(t)\right|_{L^{2}}^{2}
\end{aligned}
$$

for $0 \leq t \leq T_{1}$, where

$$
\varphi^{k+1}(t)=\left\{\begin{array}{cl}
\left|\sqrt{\rho^{k+1}} \bar{u}^{k+1}(t)\right|_{L^{2}}^{2}+\left|\bar{\rho}^{k+1}(t)\right|_{L^{2}}^{2}, & \text { if } \rho^{\infty}>0 \\
\left|\sqrt{\rho^{k+1}} \bar{u}^{k+1}(t)\right|_{L^{2}}^{2}+\left|\bar{\rho}^{k+1}(t)\right|_{L^{\frac{3}{2}} \cap L^{2}}^{2}, & \text { otherwise. }
\end{array}\right.
$$

By virtue of (4.18), (4.19) and (4.24), we deduce that

$$
\frac{d}{d t} \varphi^{k+1}(t)+\mu \psi^{k+1}(t) \leq \eta^{-1} \tilde{C} \varphi^{k+1}(t)+4 \eta \psi^{k}(t)
$$

for $0 \leq t \leq T_{1}$, where $\psi^{k+1}(t)=\left|\nabla \bar{u}^{k+1}(t)\right|_{L^{2}}^{2}$. Note that $\varphi^{k+1}(0)=0$. Hence integrating (4.25) over $(0, t)$, we have

$$
\varphi^{k+1}(t)+\mu \int_{0}^{t} \psi^{k+1}(s) d s \leq 4 \eta \int_{0}^{t} \psi^{k}(s) d s+\eta^{-1} \tilde{C} \int_{0}^{t} \varphi^{k+1}(s) d s,
$$

which implies, in view of of Gronwall's inequality, that

$$
\varphi^{k+1}(t)+\int_{0}^{t} \psi^{k+1}(s) d s \leq \eta \tilde{C} \exp \left(\eta^{-1} \tilde{C} t\right)\left(\int_{0}^{t} \psi^{k}(s) d s\right) .
$$

Choosing $\eta>0$ and then $T_{2}>0$ so small that

$$
\eta \tilde{C} \leq \frac{1}{4}, \quad T_{2}<T_{1} \quad \text { and } \quad \exp \left(\eta^{-1} \tilde{C} T_{2}\right)<2,
$$

we deduce from (4.26) that

$$
\sum_{k=1}^{\infty}\left(\sup _{0 \leq t \leq T_{2}} \varphi^{k+1}(t)+\int_{0}^{T_{2}} \psi^{k+1}(t) d t\right) \leq \tilde{C} \int_{0}^{T_{2}} \psi^{1}(t) d t<\infty .
$$

Therefore, we conclude that the sequence $\left\{\left(\rho^{k}, u^{k}\right)\right\}$ converges in a strong sense to a limit $(\rho, u)$ satisfying the regularity estimate (4.16) with $T_{*}$ replaced by $T_{2}$. Adapting the proof of Lemma 4.1, we can show that $(\rho, u)$ is a solution to the original IBVP(1.1)-(1.5) with $T$ replaced by $T_{2}$. This completes the proof of the existence. The proof of the uniqueness is similar to (indeed easier than) the proof of the convergence and so omitted. We have completed the proof of Theorem 1.1. 


\section{Proof of Theorem 1.3}

To prove Theorem 1.3, we follow basically the same methods as in the proof of Theorem 1.1. Hence we consider the linearized problem (3.1)-(3.4) with a known vector field $v$ such that

$$
\begin{gathered}
v \in C\left([0, T] ; D_{0}^{1} \cap D^{3}\right) \cap L^{2}\left(0, T ; D^{4}\right), \quad v_{t} \in L^{\infty}\left(0, T ; D_{0}^{1}\right) \cap L^{2}\left(0, T ; D^{2}\right), \\
t^{\frac{1}{2}} v \in L^{\infty}\left(0, T ; D^{4}\right), t^{\frac{1}{2}} v_{t} \in L^{\infty}\left(0, T ; D_{0}^{1} \cap D^{2}\right), t^{\frac{1}{2}} v_{t t} \in L^{2}\left(0, T ; D_{0}^{1}\right), \\
t v_{t} \in L^{\infty}\left(0, T ; D_{0}^{1} \cap D^{3}\right), \quad t v_{t t} \in L^{\infty}\left(0, T ; D_{0}^{1}\right) \cap L^{2}\left(0, T ; D^{2}\right), \\
t^{\frac{3}{2}} v_{t t} \in L^{\infty}\left(0, T ; D_{0}^{1} \cap D^{2}\right) \quad \text { and } \quad t^{\frac{3}{2}} v_{t t t} \in L^{2}\left(0, T ; D_{0}^{1}\right) .
\end{gathered}
$$

For positive initial densities and bounded domains, we have the following existence and regularity results for the linearized problem.

Lemma 5.1. Let $\Omega$ be a bounded domain in $\mathbf{R}^{3}$ with smooth boundary. Assume that $\rho_{0}, u_{0}, f, v$ and $p=p(\cdot)$ satisfy the condition (5.1) as well as the hypotheses of Theorem 1.3. If in addition, $\rho_{0} \geq \delta$ in $\Omega$ for some constant $\delta>0$, then there exists a unique solution $(\rho, u)$ to the linearized problem (3.1), (3.2) and (3.3) such that

$$
\begin{gathered}
\rho \in C\left([0, T] ; H^{3}\right), \quad \rho_{t} \in C\left([0, T] ; H^{2}\right), \quad \rho_{t t} \in L^{\infty}\left(0, T ; L^{2}\right) \cap L^{2}\left(0, T ; H^{1}\right), \\
\rho_{t t} \in L_{l o c}^{\infty}\left((0, T] ; H^{1}\right), \quad \rho_{t t t} \in L_{l o c}^{2}\left((0, T] ; L^{2}\right), \\
u \in C\left([0, T] ; H_{0}^{1} \cap H^{3}\right) \cap L^{2}\left(0, T ; H^{4}\right), \quad u_{t} \in C\left([0, T] ; H_{0}^{1}\right) \cap L^{2}\left(0, T ; H^{2}\right), \\
u_{t t} \in L^{2}\left(0, T ; L^{2}\right), \quad u \in L_{l o c}^{\infty}\left((0, T] ; H^{4}\right), \quad u_{t} \in L_{l o c}^{\infty}\left((0, T] ; H^{3}\right), \\
u_{t t} \in L_{l o c}^{\infty}\left((0, T] ; H_{0}^{1} \cap H^{2}\right), \quad u_{t t t} \in L_{l o c}^{\infty}\left((0, T] ; L^{2}\right) \cap L_{l o c}^{2}\left((0, T] ; H_{0}^{1}\right), \\
u_{t t t t} \in L_{l o c}^{2}\left((0, T] ; H^{-1}\right) \quad \text { and } \quad \rho \geq C^{-1} \delta \quad \text { on } \quad[0, T] \times \bar{\Omega} .
\end{gathered}
$$

Proof. The existence of a unique solution $\rho \in C\left([0, T] ; H^{3}\right)$ to the linear hyperbolic problem (3.1) and (3.3) was already proved in Lemma 2.1. Then the remaining regularity of $\rho$ can be derived easily from (3.1) and (5.1).

Next, if we define $F$ by $F=-\nabla p(\rho)+\rho(f-v \cdot \nabla v)$, then

$$
F \in L^{2}\left(0, T ; H^{2}\right), \quad F_{t} \in L^{2}\left(0, T ; L^{2}\right) ; \quad F \in L_{l o c}^{\infty}\left((0, T] ; H^{2}\right),
$$

$F_{t} \in L_{l o c}^{\infty}\left((0, T] ; H^{1}\right), \quad F_{t t} \in L_{l o c}^{\infty}\left((0, T] ; L^{2}\right) \quad$ and $\quad F_{t t t} \in L_{l o c}^{2}\left((0, T] ; H^{-1}\right)$.

Moreover, we observe that $\rho_{0}^{-1}\left(F(0)-L u_{0}\right)=-v(0) \cdot \nabla v(0)-g_{2} \in H_{0}^{1}$. Hence Lemma 2.2, Remark 2.3 and Lemma 2.4 allow us to deduce the existence and regularity of a unique solution $u$ to the linear parabolic problem (3.2) and (3.3). This completes the proof of Lemma 5.1.

Let $(\rho, u)$ be a solution to the linearized problem (3.1), (3.2) and (3.3) with the data $\rho_{0}, u_{0}, f, v$ and $p=p(\cdot)$ satisfying the hypotheses of Lemma 5.1. We will prove some local a priori estimates for $(\rho, u)$ which are independent of the lower bound $\delta$ of $\rho_{0}$ and the size of the domain $\Omega$.

Let us choose a constant $c_{0}>1$ so that

$$
1+\rho^{\infty}+\left|\rho_{0}-\rho^{\infty}\right|_{H^{3}}+\left|u_{0}\right|_{D_{0}^{1}}+\left|\sqrt{\rho}_{0} g_{2}\right|_{L^{2}}+\left|g_{2}\right|_{D_{0}^{1}}<c_{0} .
$$


Note that $g_{2}=\rho_{0}^{-1}\left(L u_{0}+\nabla p\left(\rho_{0}\right)\right)-f(0)=-v(0) \cdot \nabla v(0)-u_{t}(0)$. Moreover, we assume that

$$
\begin{aligned}
& |v(0)|_{D_{0}^{1} \cap D^{3}} \leq 1+c_{1}, \\
& \sup _{0 \leq t \leq T_{*}}|v(t)|_{D_{0}^{1}}+\int_{0}^{T_{*}}|v(t)|_{D^{2}}^{2} d t \leq 1+c_{2}, \\
& \sup _{0 \leq t \leq T_{*}}|v(t)|_{D^{2}}+\int_{0}^{T_{*}}\left(\left|v_{t}(t)\right|_{D_{0}^{1}}^{2}+|v(t)|_{D^{3}}^{2}\right) d s \leq 1+c_{3}, \\
& \underset{0 \leq t \leq T_{*}}{\operatorname{ess} \sup _{0}}\left(\left|v_{t}(t)\right|_{D_{0}^{1}}+|v(t)|_{D^{3}}\right)+\int_{0}^{T_{*}}\left(\left|v_{t}(t)\right|_{D^{2}}^{2}+|v(t)|_{D^{4}}^{2}\right) d t \leq 1+c_{4} \text {, }
\end{aligned}
$$

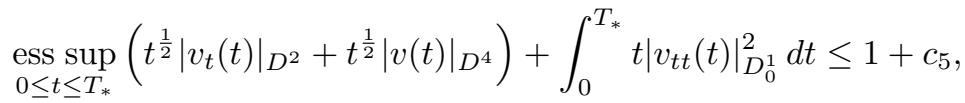

$$
\begin{aligned}
& \underset{0 \leq t \leq T_{*}}{\operatorname{ess} \sup _{0}}\left(t\left|v_{t t}(t)\right|_{D_{0}^{1}}+t\left|v_{t}(t)\right|_{D^{3}}\right)+\int_{0}^{T_{*}} t^{2}\left|v_{t t}(t)\right|_{D^{2}}^{2} d t \leq 1+c_{6}, \\
& \underset{0 \leq t \leq T_{*}}{\operatorname{ess} \sup }\left(t^{\frac{3}{2}}\left|v_{t t}(t)\right|_{D^{2}}\right)+\int_{0}^{T_{*}} t^{3}\left|v_{t t t}(t)\right|_{D_{0}^{1}}^{2} d t \leq 1+c_{6} .
\end{aligned}
$$

for some time $T_{*} \in(0, T)$ and constants $c_{i}$ 's with $1<c_{0} \leq c_{1} \leq c_{2} \leq c_{3} \leq c_{4} \leq$ $c_{5} \leq c_{6}$, which depend only on $c_{0}$ and the parameters of $C$.

Adapting the proofs of Lemma 3.2-Lemma 3.5, we can prove

\section{Lemma 5.2.}

$$
\begin{gathered}
|\rho(t)|_{L^{\infty}}+\left|\rho(t)-\rho^{\infty}\right|_{H^{3}} \leq C c_{0}, \quad\left|p(t)-p^{\infty}\right|_{H^{3}} \leq M\left(c_{0}\right), \\
\left|\rho_{t}(t)\right|_{H^{1}} \leq C c_{3}^{2}, \quad\left|p_{t}(t)\right|_{H^{1}} \leq M\left(c_{0}\right) c_{3}^{2}, \quad \int_{0}^{t}\left|\rho_{t t}(s)\right|_{L^{2}}^{2} d s \leq C c_{3}^{8}, \\
\int_{0}^{t}\left|p_{t t}(s)\right|_{L^{2}}^{2} d s \leq M\left(c_{0}\right) c_{3}^{8}, \quad\left|\rho_{t}(t)\right|_{H^{2}} \leq C c_{4}^{2}, \quad\left|p_{t}(t)\right|_{H^{2}} \leq M\left(c_{0}\right) c_{4}^{2}, \\
\left|\rho_{t t}(t)\right|_{L^{2}} \leq C c_{4}^{4}, \quad\left|p_{t t}(t)\right|_{L^{2}} \leq M\left(c_{0}\right) c_{4}^{4}, \quad \int_{0}^{t}\left|\rho_{t t}(s)\right|_{H^{1}}^{2} d s \leq C c_{4}^{8}, \\
\int_{0}^{t}\left|p_{t t}(s)\right|_{H^{1}}^{2} d s \leq M\left(c_{0}\right) c_{4}^{8}, \quad \int_{0}^{t} s\left|\rho_{t t t}(s)\right|_{L^{2}}^{2} d s \leq C c_{5}^{12}, \\
\int_{0}^{t} s\left|p_{t t t}(s)\right|_{L^{2}}^{2} d s \leq M\left(c_{0}\right) c_{5}^{12} \quad \text { and } \quad \inf _{\Omega} \rho(t) \geq C^{-1} \delta
\end{gathered}
$$

for $0 \leq t \leq \min \left(T_{*}, T_{1}\right)$, where $T_{1}=\left(1+c_{4}\right)^{-1}$ and $p^{\infty}=p\left(\rho^{\infty}\right)$.

\section{Lemma 5.3.}

$$
|u(t)|_{D_{0}^{1}}^{2}+\int_{0}^{t}|u(s)|_{D^{2}}^{2} d s \leq M\left(c_{0}\right)
$$

for $0 \leq t \leq \min \left(T_{*}, T_{2}\right)$, where $T_{2}=\left(1+c_{4}\right)^{-4}<T_{1}$. 


\section{Lemma 5.4.}

$$
\begin{gathered}
\qquad\left|\sqrt{\rho} u_{t}(t)\right|_{L^{2}}^{2}+|u(t)|_{D^{2}}^{2}+\int_{0}^{t}\left(\left|u_{t}(s)\right|_{D_{0}^{1}}^{2}+|u(s)|_{D^{3}}^{2}\right) d s \leq M\left(c_{1}\right) c_{2}^{\frac{3}{2}} c_{3}^{\frac{1}{2}}, \\
\left|u_{t}(t)\right|_{D_{0}^{1}}^{2}+|u(t)|_{D^{3}}^{2}+\int_{0}^{t}\left(\left|\sqrt{\rho} u_{t t}(s)\right|_{L^{2}}^{2}+\left|u_{t}(s)\right|_{D^{2}}^{2}+|u(s)|_{D^{4}}^{2}\right) d s \leq M\left(c_{1}\right) c_{3}^{12} \\
\text { for } 0 \leq t \leq \min \left(T_{*}, T_{3}\right), \text { where } T_{3}=\left(1+c_{4}\right)^{-9}<T_{2} .
\end{gathered}
$$

Using the same methods as in the proof of Lemma 5.4, we can derive estimates for higher regularity in positive time.

\section{Lemma 5.5.}

$$
t\left|\sqrt{\rho} u_{t t}(t)\right|_{L^{2}}^{2}+t\left|u_{t}(t)\right|_{D^{2}}^{2}+t|u(t)|_{D^{4}}^{2}+\int_{0}^{t} s\left|u_{t t}(s)\right|_{D_{0}^{1}}^{2} d s \leq M\left(c_{1}\right) c_{4}^{12}
$$

for $0 \leq t \leq \min \left(T_{*}, T_{4}\right)$, where $T_{4}=\left(1+c_{5}\right)^{-9} \leq T_{3}$.

Proof. We differentiate (3.9) with respect to $t$ again and derive

$$
\begin{aligned}
\rho u_{t t t}+L u_{t t}=-\nabla p_{t t}+\rho & (f-v \cdot \nabla v)_{t t}+2 \rho_{t}\left(f-v \cdot \nabla v-u_{t}\right)_{t} \\
& +\rho_{t t}\left(f-v \cdot \nabla v-u_{t}\right) .
\end{aligned}
$$

Multiplying this by $u_{t t}$ and integrating over $\Omega$, we have

$$
\begin{aligned}
& \frac{1}{2} \frac{d}{d t} \int \rho\left|u_{t t}\right|^{2} d x+\int \mu\left|\nabla u_{t t}\right|^{2}+(\lambda+\mu)\left(\operatorname{div} u_{t t}\right)^{2} d x \\
& =\int p_{t t} \operatorname{div} u_{t t} d x+\int \rho(f-v \cdot \nabla v)_{t t} \cdot u_{t t} d x \\
& \quad+2 \int \rho_{t}(f-v \cdot \nabla v)_{t} \cdot u_{t t} d x+\int \rho_{t t}(f-v \cdot \nabla v) \cdot u_{t t} d x \\
& \quad-\frac{3}{2} \int \rho_{t}\left|u_{t t}\right|^{2} d x-\int \rho_{t t} u_{t} \cdot u_{t t} d x
\end{aligned}
$$

Following the same arguments as in the derivation of (3.11) from (3.10), we can estimate each term of the right hand side of (5.4) as follows:

$$
\begin{gathered}
\int p_{t t} \operatorname{div} u_{t t} d x \leq C\left|p_{t t}\right|_{L^{2}}^{2}+\frac{\mu}{12}\left|\nabla u_{t t}\right|_{L^{2}}^{2}, \\
\int \rho f_{t t} \cdot u_{t t} d x \leq\left|f_{t t}\right|_{H^{-1}}\left|\rho u_{t t}\right|_{H_{0}^{1}} \leq C c_{0}^{2}\left|f_{t t}\right|_{H^{-1}}^{2}+\left|\sqrt{\rho} u_{t t}\right|_{L^{2}}^{2}+\frac{\mu}{12}\left|\nabla u_{t t}\right|_{L^{2}}^{2} \\
-\int \rho(v \cdot \nabla v)_{t t} \cdot u_{t t} d x \leq C|\rho|_{L^{\infty}}^{\frac{1}{2}}\left(|v|_{D_{0}^{1} \cap D^{2}}\left|v_{t t}\right|_{D_{0}^{1}}+\left|v_{t}\right|_{D_{0}^{1}}\left|v_{t}\right|_{D_{0}^{1} \cap D^{2}}\right)\left|\sqrt{\rho} u_{t t}\right|_{L^{2}} \\
\leq \eta^{-1} C c_{3}^{3}\left|\sqrt{\rho} u_{t t}\right|_{L^{2}}^{2}+\eta\left|v_{t t}\right|_{D_{0}^{1}}^{2}+c_{4}^{2}\left|v_{t}\right|_{D_{0}^{1} \cap D^{2}}^{2}, \\
2 \int \rho_{t}(f-v \cdot \nabla v)_{t} \cdot u_{t t} d x \leq C\left|\rho_{t}\right|_{L^{3}}\left(\left|f_{t}\right|_{L^{2}}+|v|_{D_{0}^{1} \cap D^{2}}\left|v_{t}\right|_{D_{0}^{1}}\right)\left|\nabla u_{t t}\right|_{L^{2}} \\
\leq C c_{3}^{4}\left(\left|f_{t}\right|_{L^{2}}^{2}+c_{4}^{4}\right)+\frac{\mu}{12}\left|\nabla u_{t t}\right|_{L^{2}}^{2},
\end{gathered}
$$




$$
\begin{gathered}
\int \rho_{t t}(f-v \cdot \nabla v) \cdot u_{t t} d x \leq C\left|\rho_{t t}\right|_{L^{2}}\left(|f|_{H^{1}}+|v|_{D_{0}^{1} \cap D^{2}}^{2}\right)\left|\nabla u_{t t}\right|_{L^{2}} \\
\leq C c_{3}^{4}\left|\rho_{t t}\right|_{L^{2}}^{2}+\frac{\mu}{12}\left|\nabla u_{t t}\right|_{L^{2}}^{2}, \\
-\frac{3}{2} \int \rho_{t}\left|u_{t t}\right|^{2} d x=\frac{3}{2} \int \operatorname{div}(\rho v)\left|u_{t t}\right|^{2} d x \\
\leq 3 \int \rho|v|\left|u_{t t}\right|\left|\nabla u_{t t}\right| d x \leq C c_{3}^{3}\left|\sqrt{\rho} u_{t t}\right|_{L^{2}}^{2}+\frac{\mu}{12}\left|\nabla u_{t t}\right|_{L^{2}}^{2}
\end{gathered}
$$

and finally

$$
\begin{aligned}
& -\int \rho_{t t} u_{t} \cdot u_{t t} d x \\
& \leq \int\left(\left|\rho_{t}\right||v|+\rho\left|v_{t}\right|\right)\left(\left|u_{t}\right|\left|\nabla u_{t t}\right|+\left|\nabla u_{t}\right|\left|u_{t t}\right|\right) d x \\
& \leq C\left|\rho_{t}\right|_{L^{3}}|v|_{D_{0}^{1} \cap D^{2}}\left|u_{t}\right|_{D_{0}^{1}}\left|\nabla u_{t t}\right|_{L^{2}}+C|\rho|_{L^{\infty}}^{\frac{3}{4}}\left|v_{t}\right|_{D_{0}^{1}}\left|u_{t}\right|_{D_{0}^{1}}^{\frac{1}{2}}\left|\sqrt{\rho} u_{t}\right|_{L^{2}}^{\frac{1}{2}}\left|\nabla u_{t t}\right|_{L^{2}} \\
& \quad+C|\rho|_{L^{\infty}}^{\frac{3}{4}}\left|v_{t}\right|_{D_{0}^{1}}\left|u_{t}\right|_{D_{0}^{1}}\left|\sqrt{\rho} u_{t t}\right|_{L^{2}}^{\frac{1}{2}}\left|\nabla u_{t t}\right|_{L^{2}}^{\frac{1}{2}} \\
& \leq C\left|\rho_{t}\right|_{L^{3}}^{2}|v|_{D_{0}^{1} \cap D^{2}}^{2}\left|u_{t}\right|_{D_{0}^{1}}^{2}+C|\rho|_{L^{\infty}}^{\frac{3}{2}}\left|v_{t}\right|_{D_{0}^{1}}^{2}\left|u_{t}\right|_{D_{0}^{1}}\left|\sqrt{\rho} u_{t}\right|_{L^{2}} \\
& \quad+C|\rho|_{L^{\infty}}^{\frac{3}{2}}\left|v_{t}\right|_{D_{0}^{1}}^{2}\left|u_{t}\right|_{D_{0}^{1}}^{2}+C\left|\sqrt{\rho} u_{t t}\right|_{L^{2}}^{2}+\frac{\mu}{12}\left|\nabla u_{t t}\right|_{L^{2}}^{2} \\
& \leq C c_{4}^{7}\left|u_{t}\right|_{D_{0}^{1}}^{2}+\left|\sqrt{\rho} u_{t}\right|_{L^{2}}^{2}+C\left|\sqrt{\rho} u_{t t}\right|_{L^{2}}^{2}+\frac{\mu}{12}\left|\nabla u_{t t}\right|_{L^{2}}^{2} .
\end{aligned}
$$

Substituting all the estimates into (5.4) and taking $\eta=\left(1+c_{5}\right)^{-1}$, we have

$$
\begin{aligned}
& \frac{d}{d t} \int \rho\left|u_{t t}\right|^{2} d x+\mu \int\left|\nabla u_{t t}\right|^{2} d x \\
& \leq C\left(c_{0}^{2}\left|f_{t t}\right|_{H^{-1}}^{2}+c_{3}^{4}\left|f_{t}\right|_{L^{2}}^{2}+c_{4}^{8}+\left|p_{t t}\right|_{L^{2}}^{2}+c_{3}^{4}\left|\rho_{t t}\right|_{L^{2}}^{2}+c_{4}^{2}\left|v_{t}\right|_{D_{0}^{1} \cap D^{2}}^{2}\right) \\
& \quad+C\left(c_{4}^{7}\left|u_{t}\right|_{D_{0}^{1}}^{2}+\left|\sqrt{\rho} u_{t}\right|_{L^{2}}^{2}\right)+\left(1+c_{5}\right)^{-1}\left|v_{t t}\right|_{D_{0}^{1}}^{2}+C c_{5}^{4}\left|\sqrt{\rho} u_{t t}\right|_{L^{2}}^{2}
\end{aligned}
$$

for $0 \leq t \leq \min \left(T_{*}, T_{3}\right)$. From Lemma 5.2 and Lemma 5.4, we observe that

$$
t^{\frac{1}{2}}\left|f_{t t}(t)\right|_{H^{-1}} \in L^{2}(0, T), \quad t^{\frac{1}{2}}\left|v_{t t}(t)\right|_{D_{0}^{1}} \in L^{2}\left(0, T_{*}\right)
$$

and all the remaining terms in the right hand side of $(5.5)$ are integrable in $\left(0, \min \left(T_{*}, T_{3}\right)\right)$. Hence multiplying (5.5) by $t$ and integrating over $(\tau, \bar{t})$, we obtain

$$
\begin{aligned}
& \bar{t}\left|\sqrt{\rho} u_{t t}(\bar{t})\right|_{L^{2}}^{2}+\mu \int_{\tau}^{\bar{t}} t\left|\nabla u_{t t}(t)\right|_{L^{2}}^{2} d t \\
& \leq M\left(c_{1}\right) c_{4}^{12}+\tau\left|\sqrt{\rho} u_{t t}(\tau)\right|_{L^{2}}^{2}+\int_{\tau}^{\bar{t}} C c_{5}^{4} t\left|\sqrt{\rho} u_{t t}(t)\right|_{L^{2}}^{2} d t
\end{aligned}
$$

for $0<\tau \leq \bar{t} \leq \min \left(T_{*}, T_{3}\right)$. By virtue of Gronwall's inequality, we deduce that

$$
t\left|\sqrt{\rho} u_{t t}(t)\right|_{L^{2}}^{2}+\int_{\tau}^{t} s\left|\nabla u_{t t}(s)\right|_{L^{2}}^{2} d s \leq M\left(c_{1}\right)\left(c_{4}^{12}+\tau\left|\sqrt{\rho} u_{t t}(\tau)\right|_{L^{2}}^{2}\right)
$$

for $0<\tau \leq t \leq \min \left(T_{*}, T_{4}\right)$, where $T_{4}=\left(1+c_{5}\right)^{-9} \leq T_{3}$. On the other hand, since $\sqrt{\rho} u_{t t} \in L^{2}\left(0, T ; L^{2}\right)$, it follows (see also Remark 5 in [1]) that there is a sequence 
$\left\{\tau_{k}\right\}$ of positive times such that

$$
\tau_{k} \rightarrow 0 \quad \text { and } \quad \tau_{k}\left|\sqrt{\rho} u_{t t}\left(\tau_{k}\right)\right|_{L^{2}}^{2} \rightarrow 0 \quad \text { as } \quad k \rightarrow \infty .
$$

Therefore, letting $\tau=\tau_{k} \rightarrow 0$ in (5.6), we conclude that

$$
t\left|\sqrt{\rho} u_{t t}(t)\right|_{L^{2}}^{2}+\int_{0}^{t} s\left|u_{t t}(s)\right|_{D_{0}^{1}}^{2} d s \leq M\left(c_{1}\right) c_{4}^{12}
$$

for $0<t \leq \min \left(T_{*}, T_{4}\right)$. Moreover, since

$$
L u=-\nabla p+\rho\left(f-v \cdot \nabla v-u_{t}\right)
$$

and

$$
L u_{t}=-\nabla p_{t}+\rho\left(f-v \cdot \nabla v-u_{t}\right)_{t}+\rho_{t}\left(f-v \cdot \nabla v-u_{t}\right),
$$

it follows from the elliptic regularity result that

$$
t\left|u_{t}(t)\right|_{D^{2}}^{2}+t|u(t)|_{D^{4}}^{2} \leq M\left(c_{1}\right) c_{4}^{12} \quad \text { for } \quad 0 \leq t \leq \min \left(T_{*}, T_{4}\right) .
$$

This completes the proof of Lemma 5.5.

\section{Lemma 5.6.}

$$
t^{2}\left|u_{t t}(t)\right|_{D_{0}^{1}}^{2}+t^{2}\left|u_{t}(t)\right|_{D^{3}}^{2}+\int_{0}^{t} s^{2}\left(\left|\sqrt{\rho} u_{t t t}(s)\right|_{L^{2}}^{2}+\left|u_{t t}(s)\right|_{D^{2}}^{2}\right) d s \leq M\left(c_{1}\right) c_{5}^{17}
$$

for $0 \leq t \leq \min \left(T_{*}, T_{4}\right)$.

Proof. Multiplying (5.3) by $u_{t t t}$ and integrating over $\Omega$, we have

$$
\begin{array}{r}
\int \rho\left|u_{t t t}\right|^{2} d x+\frac{1}{2} \frac{d}{d t} \int \mu\left|\nabla u_{t t}\right|^{2}+(\lambda+\mu)\left(\operatorname{div} u_{t t}\right)^{2} d x \\
=\int\left(-\nabla p_{t t}+\rho(f-v \cdot \nabla v)_{t t}\right) \cdot u_{t t t} d x \\
+\int 2 \rho_{t}\left(f-v \cdot \nabla v-u_{t}\right)_{t} \cdot u_{t t t} d x \\
\quad+\int \rho_{t t}\left(f-v \cdot \nabla v-u_{t}\right) \cdot u_{t t t} d x
\end{array}
$$

We easily estimate the first term of the right hand side in (5.7) as follows.

$$
\begin{aligned}
-\int \nabla p_{t t} \cdot u_{t t t} d x & =\frac{d}{d t} \int p_{t t} \operatorname{div} u_{t t} d x-\int p_{t t t} \operatorname{div} u_{t t} d x \\
& \leq \frac{d}{d t} \int p_{t t} \operatorname{div} u_{t t} d x+\left|p_{t t t}\right|_{L^{2}}^{2}+\left|u_{t t}\right|_{D_{0}^{1}}^{2}
\end{aligned}
$$

and

$$
\begin{aligned}
& \int \rho(f-v \cdot \nabla v)_{t t} \cdot u_{t t t} d x \\
& \quad \leq C|\rho|_{L^{\infty}}^{\frac{1}{2}}\left|(f-v \cdot \nabla v)_{t t}\right|_{L^{2}}\left|\sqrt{\rho} u_{t t t}\right|_{L^{2}} \\
& \quad \leq C|\rho|_{L^{\infty}}^{\frac{1}{2}}\left(\left|f_{t t}\right|_{L^{2}}+|v|_{D_{0}^{1} \cap D^{2}}\left|v_{t t}\right|_{D_{0}^{1}}+\left|v_{t}\right|_{D_{0}^{1}}\left|v_{t}\right|_{D_{0}^{1} \cap D^{2}}\right)\left|\sqrt{\rho} u_{t t t}\right|_{L^{2}} \\
& \quad \leq C c_{4}^{3}\left(\left|f_{t t}\right|_{L^{2}}^{2}+\left|v_{t t}\right|_{D_{0}^{1}}^{2}+\left|v_{t}\right|_{D_{0}^{1} \cap D^{2}}^{2}\right)+\frac{1}{2}\left|\sqrt{\rho} u_{t t t}\right|_{L^{2}}^{2} .
\end{aligned}
$$


To estimate the second term, we observe that

$$
\begin{aligned}
\int \rho_{t} & (f-v \cdot \nabla v)_{t} \cdot u_{t t t} d x \\
= & \frac{d}{d t} \int \rho_{t}(f-v \cdot \nabla v)_{t} \cdot u_{t t} d x-\int \rho_{t t}(f-v \cdot \nabla v)_{t} \cdot u_{t t} d x \\
& -\int \rho_{t}(f-v \cdot \nabla v)_{t t} \cdot u_{t t} d x
\end{aligned}
$$

and

$$
-\int \rho_{t} u_{t t} \cdot u_{t t t} d x=-\frac{d}{d t} \int \rho_{t}\left(\frac{1}{2}\left|u_{t t}\right|^{2}\right) d x+\int \rho_{t t}\left(\frac{1}{2}\left|u_{t t}\right|^{2}\right) d x
$$

But in view of Lemma 5.2, we obtain

$$
\begin{aligned}
-\int \rho_{t t}(f-v \cdot \nabla v)_{t} \cdot u_{t t} d x & \leq C\left|\rho_{t t}\right|_{L^{2}}\left(\left|f_{t}\right|_{H^{1}}+|v|_{D_{0}^{1} \cap D^{2}}\left|v_{t}\right|_{D_{0}^{1} \cap D^{2}}\right)\left|u_{t t}\right|_{D_{0}^{1}} \\
& \leq C c_{4}^{5}\left(\left|f_{t}\right|_{H^{1}}^{2}+\left|v_{t}\right|_{D_{0}^{1} \cap D^{2}}^{2}+\left|u_{t t}\right|_{D_{0}^{1}}^{2}\right) \\
-\int \rho_{t}(f-v \cdot \nabla v)_{t t} \cdot u_{t t} d x & \leq\left. C\left|\rho_{t}\right|_{L^{3}}\left|(f-v \cdot \nabla v)_{t t}\right|\right|_{L^{2}}\left|u_{t t}\right|_{D_{0}^{1}} \\
& \leq C c_{4}^{4}\left(\left|f_{t t}\right|_{L^{2}}^{2}+\left|v_{t t}\right|_{D_{0}^{1}}^{2}+\left|v_{t}\right|_{D_{0}^{1} \cap D^{2}}^{2}+\left|u_{t t}\right|_{D_{0}^{1}}^{2}\right)
\end{aligned}
$$

and

$$
\begin{aligned}
\int \rho_{t t}\left(\frac{1}{2}\left|u_{t t}\right|^{2}\right) d x & =-\int \operatorname{div}\left((\rho v)_{t}\right)\left(\frac{1}{2}\left|u_{t t}\right|^{2}\right) d x \\
& \leq \int\left(\left|\rho_{t}\right||v|+\rho\left|v_{t}\right|\right)\left|u_{t t}\right|\left|\nabla u_{t t}\right| d x \\
& \leq C c_{3}^{3}\left|u_{t t}\right|_{D_{0}^{1}}^{2}+C\left|v_{t}\right|_{D_{0}^{1} \cap D^{2}}^{2}\left|\sqrt{\rho} u_{t t}\right|_{L^{2}}^{2} .
\end{aligned}
$$

Similarly, we can estimate the last term as follows.

$$
\begin{aligned}
& \int \rho_{t t}(f-v \cdot \nabla v) \cdot u_{t t t} d x \\
& =\frac{d}{d t} \int \rho_{t t}(f-v \cdot \nabla v) \cdot u_{t t} d x-\int\left(\rho_{t t}(f-v \cdot \nabla v)\right)_{t} \cdot u_{t t} d x \\
& \leq \frac{d}{d t} \int \rho_{t t}(f-v \cdot \nabla v) \cdot u_{t t} d x \\
& \quad+C c_{4}^{5}\left(\left|\rho_{t t t}\right|_{L^{2}}^{2}+\left|f_{t}\right|_{H^{1}}^{2}+\left|v_{t}\right|_{D_{0}^{1} \cap D^{2}}^{2}+\left|u_{t t}\right|_{D_{0}^{1}}^{2}\right)
\end{aligned}
$$


and

$$
\begin{aligned}
-\int & \rho_{t t} u_{t} \cdot u_{t t t} d x \\
=- & \frac{d}{d t} \int \rho_{t t} u_{t} \cdot u_{t t} d x+\int\left(\rho_{t t t} u_{t}+\rho_{t t} u_{t t}\right) \cdot u_{t t} d x \\
=- & \frac{d}{d t} \int \rho_{t t} u_{t} \cdot u_{t t} d x-\int \operatorname{div}\left((\rho v)_{t t}\right) u_{t} \cdot u_{t t} d x+\int \rho_{t t}\left|u_{t t}\right|^{2} d x \\
\leq- & \frac{d}{d t} \int \rho_{t t} u_{t} \cdot u_{t t} d x+C c_{4}^{5}\left(\left|u_{t}\right|_{D_{0}^{1} \cap D^{2}}^{2}+\left|u_{t t}\right|_{D_{0}^{1}}^{2}\right) \\
\quad & \quad+C\left(\left|v_{t}\right|_{D_{0}^{1} \cap D^{2}}^{2}+\left|v_{t t}\right|_{D_{0}^{1}}^{2}\right)\left(\left|\sqrt{\rho} u_{t}\right|_{L^{2}}^{2}+\left|u_{t}\right|_{D_{0}^{1}}^{2}+\left|\sqrt{\rho} u_{t t}\right|_{L^{2}}^{2}\right) .
\end{aligned}
$$

Substituting all the above estimates into (5.7), we have

$$
\begin{aligned}
& \frac{1}{2} \int \rho\left|u_{t t t}\right|^{2} d x+\frac{1}{2} \frac{d}{d t} \int \mu\left|\nabla u_{t t}\right|^{2}+(\lambda+\mu)\left(\operatorname{div} u_{t t}\right)^{2} d x \\
& \leq \frac{d}{d t} \Lambda_{1}+C c_{4}^{5}\left(\left|p_{t t t}\right|_{L^{2}}^{2}+\left|\rho_{t t t}\right|_{L^{2}}^{2}+\left|f_{t t}\right|_{L^{2}}^{2}+\left|f_{t}\right|_{H^{1}}^{2}\right) \\
& \quad+C c_{4}^{5}\left(\left|v_{t t}\right|_{D_{0}^{1}}^{2}+\left|v_{t}\right|_{D_{0}^{1} \cap D^{2}}^{2}+\left|u_{t}\right|_{D_{0}^{1} \cap D^{2}}^{2}+\left|u_{t t}\right|_{D_{0}^{1}}^{2}\right) \\
& \quad+C\left(\left|v_{t}\right|_{D_{0}^{1} \cap D^{2}}^{2}+\left|v_{t t}\right|_{D_{0}^{1}}^{2}\right)\left(\left|\sqrt{\rho} u_{t}\right|_{L^{2}}^{2}+\left|u_{t}\right|_{D_{0}^{1}}^{2}+\left|\sqrt{\rho} u_{t t}\right|_{L^{2}}^{2}\right)
\end{aligned}
$$

for $0 \leq t \leq \min \left(T_{*}, T_{4}\right)$, where

$$
\begin{aligned}
\Lambda_{1}(t)=\int\left(p_{t t} \operatorname{div} u_{t t}\right. & \left.+2 \rho_{t}(f-v \cdot \nabla v)_{t} \cdot u_{t t}+\rho_{t t}(f-v \cdot \nabla v) \cdot u_{t t}\right)(t) d x \\
& -\int\left(\rho_{t}\left|u_{t t}\right|^{2}+\rho_{t t} u_{t} \cdot u_{t t}\right)(t) d x .
\end{aligned}
$$

Hence if we multiply this by $t^{2}$ and integrate over $(\tau, \bar{t})$, then by virtue of the previous lemmas, we deduce that

$$
\begin{aligned}
\frac{1}{2} \int_{\tau}^{\bar{t}} t^{2}\left|\sqrt{\rho} u_{t t t}(t)\right|_{L^{2}}^{2} d t & +\frac{\mu}{2} \bar{t}^{2}\left|\nabla u_{t t}(\bar{t})\right|_{L^{2}}^{2} \\
\leq M\left(c_{1}\right) c_{5}^{17} & +C \tau^{2}\left|\nabla u_{t t}(\tau)\right|_{L^{2}}^{2}+\left|\bar{t}^{2} \Lambda_{1}(\bar{t})\right| \\
& +\left|\tau^{2} \Lambda_{1}(\tau)\right|+C \int_{\tau}^{\bar{t}} t\left|\Lambda_{1}(t)\right| d t
\end{aligned}
$$

for $0<\tau \leq \bar{t} \leq \min \left(T_{*}, T_{4}\right)$. It is easy to show that

$$
\left|\Lambda_{1}(t)\right| \leq t^{-1} M\left(c_{1}\right) c_{4}^{14}+\frac{\mu}{4}\left|\nabla u_{t t}(t)\right|_{L^{2}}^{2} \quad \text { for } \quad 0 \leq t \leq \min \left(T_{*}, T_{4}\right) .
$$

Therefore, recalling that

$$
\int_{0}^{t} s\left|\nabla u_{t t}(s)\right|_{L^{2}}^{2} d s \leq M\left(c_{1}\right) c_{4}^{12} \quad \text { for } \quad 0 \leq t \leq \min \left(T_{*}, T_{4}\right)
$$

and

$$
\tau_{k}^{2}\left|\nabla u_{t t}\left(\tau_{k}\right)\right|_{L^{2}}^{2} \rightarrow 0 \quad \text { for some sequence } \quad\left\{\tau_{k}\right\} \quad \text { with } \quad \tau_{k} \rightarrow 0
$$


we conclude from (5.8) that

$$
\int_{0}^{t} s^{2}\left|\sqrt{\rho} u_{t t t}(s)\right|_{L^{2}}^{2} d s+t^{2}\left|\nabla u_{t t}(t)\right|_{L^{2}}^{2} \leq M\left(c_{1}\right) c_{5}^{17}
$$

for $0 \leq t \leq \min \left(T_{*}, T_{4}\right)$. Then in view of the elliptic regularity result, we complete the proof of Lemma 5.6.

\section{Lemma 5.7.}

$$
t^{3}\left|\sqrt{\rho} u_{t t t}(t)\right|_{L^{2}}^{2}+t^{3}\left|u_{t t}(t)\right|_{D^{2}}^{2}+\int_{0}^{t} s^{3}\left|u_{t t t}(s)\right|_{D_{0}^{1}}^{2} d s \leq M\left(c_{1}\right) c_{5}^{22}
$$

for $0 \leq t \leq \min \left(T_{*}, T_{5}\right)$, where $T_{5}=\left(1+c_{6}\right)^{-9} \leq T_{4}$.

Proof. Differentiating (5.3) with respect to $t$ again, we derive

$$
\begin{array}{r}
\rho u_{t t t t}+L u_{t t t}=-\nabla p_{t t t}+\rho(f-v \cdot \nabla v)_{t t t}+3 \rho_{t}\left(f-v \cdot \nabla v-u_{t}\right)_{t t} \\
+3 \rho_{t t}\left(f-v \cdot \nabla v-u_{t}\right)_{t}+\rho_{t t t}\left(f-v \cdot \nabla v-u_{t}\right) .
\end{array}
$$

Multiplying this by $u_{t t t}$ and integrating over $\Omega$, we have

$$
\begin{aligned}
\frac{1}{2} \frac{d}{d t} \int \rho\left|u_{t t t}\right|^{2} d x+\int \mu\left|\nabla u_{t t t}\right|^{2}+(\lambda+\mu)\left(\operatorname{div} u_{t t t}\right)^{2} d x \\
=\int\left(p_{t t t} \operatorname{div} u_{t t t}+\rho(f-v \cdot \nabla v)_{t t t} \cdot u_{t t}\right) d x \\
\quad+3 \int \rho_{t}(f-v \cdot \nabla v)_{t t} \cdot u_{t t t} d x-\frac{5}{2} \int \rho_{t}\left|u_{t t t}\right|^{2} d x \\
\quad+3 \int \rho_{t t}(f-v \cdot \nabla v)_{t} \cdot u_{t t t} d x-3 \int \rho_{t t} u_{t t} \cdot u_{t t t} d x \\
\quad+\int \rho_{t t t}(f-v \cdot \nabla v) \cdot u_{t t t} d x-\int \rho_{t t t} u_{t} \cdot u_{t t t} d x
\end{aligned}
$$

Using Lemma 5.2 and Lemma 5.4 , we can estimate each term of the right hand side of (5.10) as follows:

$$
\begin{aligned}
& \int p_{t t t} \operatorname{div} u_{t t t} d x \leq C\left|p_{t t t}\right|_{L^{2}}^{2}+\frac{\mu}{16}\left|\nabla u_{t t t}\right|_{L^{2}}^{2}, \\
& \int \rho f_{t t t} \cdot u_{t t t} d x \leq\left|f_{t t t}\right|_{H^{-1}}\left|\rho u_{t t t}\right|_{D_{0}^{1}} \leq C c_{0}^{2}\left|f_{t t t}\right|_{H^{-1}}^{2}+\left|\sqrt{\rho} u_{t t t}\right|_{L^{2}}^{2}+\frac{\mu}{16}\left|\nabla u_{t t t}\right|_{L^{2}}^{2}, \\
& -\int \rho(v \cdot \nabla v)_{t t t} \cdot u_{t t t} d x \\
& \leq C|\rho|_{L^{\infty}}^{\frac{1}{2}}\left(|v|_{D_{0}^{1} \cap D^{2}}\left|v_{t t t}\right|_{D_{0}^{1}}+\left|v_{t}\right|_{D_{0}^{1} \cap D^{2}}\left|v_{t t}\right|_{D_{0}^{1}}\right)\left|\sqrt{\rho} u_{t t t}\right|_{L^{2}} \\
& \leq \eta^{-1} C c_{3}^{3}\left|\sqrt{\rho} u_{t t t}\right|_{L^{2}}^{2}+\eta\left|v_{t t t}\right|_{D_{0}^{1}}^{2}+\left|v_{t}\right|_{D_{0}^{1} \cap D^{2}}^{2}\left|v_{t t}\right|_{D_{0}^{1}}^{2}, \\
& 3 \int \rho_{t}(f-v \cdot \nabla v)_{t t} \cdot u_{t t t} d x \\
& \leq C\left|\rho_{t}\right|_{L^{3}}\left(\left|f_{t t}\right|_{L^{2}}+|v|_{D_{0}^{1} \cap D^{2}}\left|v_{t t}\right|_{D_{0}^{1}}+\left|v_{t}\right|_{D_{0}^{1}}\left|v_{t}\right|_{D_{0}^{1} \cap D^{2}}\right)\left|\nabla u_{t t t}\right|_{L^{2}} \\
& \leq C c_{4}^{6}\left(\left|f_{t t}\right|_{L^{2}}^{2}+\left|v_{t t}\right|_{D_{0}^{1}}^{2}+\left|v_{t}\right|_{D_{0}^{1} \cap D^{2}}\right)+\frac{\mu}{16}\left|\nabla u_{t t}\right|_{L^{2}}^{2},
\end{aligned}
$$




$$
\begin{aligned}
& -\frac{5}{2} \int \rho_{t}\left|u_{t t t}\right|^{2} d x=\frac{5}{2} \int \operatorname{div}(\rho v)\left|u_{t t t}\right|^{2} d x \leq C \int \rho|v|\left|u_{t t t}\right|\left|\nabla u_{t t t}\right| d x \\
& \leq C c_{3}^{3}\left|\sqrt{\rho} u_{t t t}\right|_{L^{2}}^{2}+\frac{\mu}{16}\left|\nabla u_{t t t}\right|_{L^{2}}^{2}, \\
& 3 \int \rho_{t t}(f-v \cdot \nabla v)_{t} \cdot u_{t t t} d x \\
& \leq C\left|\rho_{t t}\right|_{L^{2}}\left(\left|f_{t}\right|_{H^{1}}+|v|_{D_{0}^{1} \cap D^{2}}\left|v_{t}\right|_{D_{0}^{1} \cap D^{2}}\right)\left|\nabla u_{t t t}\right|_{L^{2}} \\
& \leq C c_{4}^{10}\left(\left|f_{t}\right|_{H^{1}}^{2}+\left|v_{t}\right|_{D_{0}^{1} \cap D^{2}}^{2}\right)+\frac{\mu}{16}\left|\nabla u_{t t}\right|_{L^{2}}^{2}, \\
& -3 \int \rho_{t t} u_{t t} \cdot u_{t t t} d x \\
& \leq C \int\left(\left|\rho_{t}\right||v|+\rho\left|v_{t}\right|\right)\left(\left|u_{t t}\right|\left|\nabla u_{t t t}\right|+\left|\nabla u_{t t}\right|\left|u_{t t t}\right|\right) d x \\
& \leq C\left|\rho_{t}\right|_{L^{3}}|v|_{D_{0}^{1} \cap D^{2}}\left|u_{t t}\right|_{D_{0}^{1}}\left|\nabla u_{t t t}\right|_{L^{2}}+C|\rho|_{L^{\infty}}^{\frac{3}{4}}\left|v_{t}\right|_{D_{0}^{1}}\left|u_{t t}\right|_{D_{0}^{1}}^{\frac{1}{2}}\left|\sqrt{\rho} u_{t t}\right|_{L^{2}}^{\frac{1}{2}}\left|\nabla u_{t t t}\right|_{L^{2}} \\
& +C|\rho|_{L^{\infty}}^{\frac{3}{4}}\left|v_{t}\right|_{D_{0}^{1}}\left|u_{t t}\right|_{D_{0}^{1}}\left|\sqrt{\rho} u_{t t t}\right|_{L^{2}}^{\frac{1}{2}}\left|\nabla u_{t t t}\right|_{L^{2}}^{\frac{1}{2}} \\
& \leq C c_{4}^{7}\left|u_{t t}\right|_{D_{0}^{1}}^{2}+\left|\sqrt{\rho} u_{t t}\right|_{L^{2}}^{2}+C\left|\sqrt{\rho} u_{t t t}\right|_{L^{2}}^{2}+\frac{\mu}{16}\left|\nabla u_{t t t}\right|_{L^{2}}^{2}, \\
& \int \rho_{t t t}(f-v \cdot \nabla v) \cdot u_{t t t} d x \leq C\left|\rho_{t t t}\right|_{L^{2}}\left(|f|_{H^{1}}+|v|_{D_{0}^{1} \cap D^{2}}^{2}\right)\left|\nabla u_{t t t}\right|_{L^{2}} \\
& \leq C c_{3}^{4}\left|\rho_{t t t}\right|_{L^{2}}^{2}+\frac{\mu}{16}\left|\nabla u_{t t t}\right|_{L^{2}}^{2}
\end{aligned}
$$

and finally

$$
\begin{aligned}
& -\int \rho_{t t t} u_{t} \cdot u_{t t t} d x \\
& \leq C \int\left(\left|\rho_{t t}\right||v|+\left|\rho_{t}\right|\left|v_{t}\right|+\rho\left|v_{t t}\right|\right)\left(\left|u_{t}\right|\left|\nabla u_{t t t}\right|+\left|\nabla u_{t}\right|\left|u_{t t t}\right|\right) d x \\
& \leq C\left(\left|\rho_{t t}\right|_{L^{2}}|v|_{D_{0}^{1} \cap D^{2}}+\left|\rho_{t}\right|_{H^{1}}\left|v_{t}\right|_{D_{0}^{1}}\right)\left|u_{t}\right|_{D_{0}^{1} \cap D^{2}}\left|\nabla u_{t t t}\right|_{L^{2}} \\
& \quad+C|\rho|_{L^{\infty}}^{\frac{3}{4}}\left|v_{t t}\right|_{D_{0}^{1}}\left|\sqrt{\rho} u_{t}\right|_{L^{2}}^{\frac{1}{2}}\left|u_{t}\right|_{D_{0}^{1}}^{\frac{1}{2}}\left|\nabla u_{t t t}\right|_{L^{2}} \\
& \quad+C|\rho|_{L^{\infty}}^{\frac{3}{4}}\left|v_{t t}\right|_{D_{0}^{1}}\left|u_{t}\right|_{D_{0}^{1}}\left|\sqrt{\rho} u_{t t t}\right|_{L^{2}}^{\frac{1}{2}}\left|\nabla u_{t t t}\right|_{L^{2}}^{\frac{1}{2}} \\
& \leq C c_{4}^{10}\left|u_{t}\right|_{D_{0}^{1} \cap D^{2}}^{2}+M\left(c_{1}\right) c_{3}^{10}\left|v_{t t}\right|_{D_{0}^{1}}^{2}+C\left|\sqrt{\rho} u_{t t t}\right|_{L^{2}}^{2}+\frac{\mu}{16}\left|\nabla u_{t t t}\right|_{L^{2}}^{2} .
\end{aligned}
$$

Substituting all the estimates into (5.10) and choosing $\eta=\left(1+c_{6}\right)^{-1}$, we have

$$
\begin{aligned}
& \frac{d}{d t} \int \rho\left|u_{t t t}\right|^{2} d x+\mu \int\left|\nabla u_{t t t}\right|^{2} d x \\
& \leq \\
& \quad C c_{4}^{10}\left(\left|p_{t t t}\right|_{L^{2}}^{2}+\left|\rho_{t t t}\right|_{L^{2}}^{2}+\left|f_{t t t}\right|_{H^{-1}}^{2}+\left|f_{t t}\right|_{L^{2}}^{2}+\left|f_{t}\right|_{H^{1}}^{2}\right) \\
& \quad+M\left(c_{1}\right) c_{4}^{10}\left(\left|v_{t}\right|_{D_{0}^{1} \cap D^{2}}^{2}+\left|v_{t t}\right|_{D_{0}^{1}}^{2}+\left|v_{t}\right|_{D_{0}^{1} \cap D^{2}}\left|v_{t t}\right|_{D_{0}^{1}}^{2}\right)+\left(1+c_{6}\right)^{-1}\left|v_{t t t}\right|_{D_{0}^{1}}^{2} \\
& \quad+C c_{4}^{10}\left(\left|u_{t t}\right|_{D_{0}^{1}}^{2}+\left|u_{t}\right|_{D_{0}^{1} \cap D^{2}}^{2}+\left|\sqrt{\rho} u_{t t}\right|_{L^{2}}^{2}\right)+C c_{6}^{4}\left|\sqrt{\rho} u_{t t t}\right|_{L^{2}}^{2}
\end{aligned}
$$


for $0 \leq t \leq \min \left(T_{*}, T_{4}\right)$. Hence multiplying this by $t^{3}$, integrating over $(0, \bar{t})$ and using Lemma 5.2-Lemma 5.5, we deduce that

$$
\begin{aligned}
\bar{t}^{3}\left|\sqrt{\rho} u_{t t t}(\bar{t})\right|_{L^{2}}^{2} & +\int_{0}^{\bar{t}} t^{3}\left|u_{t t t}(t)\right|_{D_{0}^{1}}^{2} d t \\
& \leq M\left(c_{1}\right) c_{5}^{22}+\int_{0}^{\bar{t}} C c_{6}^{4} t^{3}\left|\sqrt{\rho} u_{t t t}(t)\right|_{L^{2}}^{2} d t
\end{aligned}
$$

for $0 \leq \bar{t} \leq \min \left(T_{*}, T_{4}\right)$. Therefore, in view of Gronwall's inequality, we conclude that

$$
t\left|\sqrt{\rho} u_{t t t}(t)\right|_{L^{2}}^{2}+\int_{0}^{t} s^{3}\left|u_{t t t}(s)\right|_{D_{0}^{1}}^{2} d s \leq M\left(c_{1}\right) c_{5}^{22}
$$

for $0 \leq t \leq \min \left(T_{*}, T_{5}\right)$, where $T_{5}=\left(1+c_{6}\right)^{-9} \leq T_{4}$. Then by virtue of the elliptic regularity result, we complete the proof of Lemma 5.7.

Combining all the previous lemmas, we obtain

$$
\begin{aligned}
& |u(t)|_{D_{0}^{1}}+\int_{0}^{t}|u(s)|_{D^{2}}^{2} d s \leq M\left(c_{1}\right), \\
& |u(t)|_{D^{2}}+\int_{0}^{t}\left(\left|u_{t}(s)\right|_{D_{0}^{1}}^{2}+|u(s)|_{D^{3}}^{2}\right) d s \leq M\left(c_{1}\right) c_{2}^{\frac{3}{2}} c_{3}^{\frac{1}{2}}, \\
& \left|u_{t}(t)\right|_{D_{0}^{1}}+|u(t)|_{D^{3}}+\int_{0}^{t}\left(\left|u_{t}(s)\right|_{D^{2}}^{2}+|u(s)|_{D^{4}}^{2}\right) d s \leq M\left(c_{1}\right) c_{3}^{12}, \\
& t^{\frac{1}{2}}\left|u_{t}(t)\right|_{D^{2}}+t^{\frac{1}{2}}|u(t)|_{D^{4}}+\int_{0}^{t} s\left|u_{t t}(s)\right|_{D_{0}^{1}}^{2} d s \leq M\left(c_{1}\right) c_{4}^{12}, \\
& t\left|u_{t t}(t)\right|_{D_{0}^{1}}+t\left|u_{t}(t)\right|_{D^{3}}+\int_{0}^{t} s^{2}\left|u_{t t}(t)\right|_{D^{2}}^{2} d t \leq M\left(c_{1}\right) c_{5}^{22}, \\
& t^{\frac{3}{2}}\left|u_{t t}(t)\right|_{D^{2}}+\int_{0}^{t} s^{3}\left|u_{t t t}(s)\right|_{D_{0}^{1}}^{2} d t \leq M\left(c_{1}\right) c_{5}^{22}, \\
& \left|\rho(t)-\rho^{\infty}\right|_{H^{3}}+\left|\rho_{t}(t)\right|_{H^{2}}+\left|\sqrt{\rho} u_{t}(t)\right|_{L^{2}}+\int_{0}^{t}\left|\sqrt{\rho} u_{t t}(s)\right|_{L^{2}}^{2} d s \leq M\left(c_{1}\right) c_{5}^{22}, \\
& t^{\frac{1}{2}}\left|\sqrt{\rho} u_{t t}\right|_{L^{2}}+t^{\frac{3}{2}}\left|\sqrt{\rho} u_{t t t}(t)\right|_{L^{2}}+\int_{0}^{t} s^{2}\left|\sqrt{\rho} u_{t t t}(s)\right|_{L^{2}}^{2} d s \leq M\left(c_{1}\right) c_{5}^{22} .
\end{aligned}
$$

for $0 \leq t \leq \min \left(T_{*}, T_{5}\right)$, where $T_{5}=\left(1+c_{6}\right)^{-9}$. Therefore, if we define the constants $c_{i}$ and $T_{*} \in(0,1)$ by

$$
\begin{gathered}
c_{1}=M\left(c_{0}\right), \quad c_{2}=M\left(c_{1}\right), \quad c_{3}=c_{2}^{5}, \quad c_{4}=c_{2} c_{3}^{12}, \\
c_{5}=c_{2} c_{4}^{12}, \quad c_{6}=c_{2} c_{5}^{22} \quad \text { and } \quad T_{*}=\min \left(T,\left(1+c_{6}\right)^{-9}\right),
\end{gathered}
$$


then we conclude that

$$
\begin{aligned}
& \sup _{0 \leq t \leq T_{*}}|u(t)|_{D_{0}^{1}}+\int_{0}^{T_{*}}|u(t)|_{D^{2}}^{2} d t \leq c_{2}, \\
& \sup _{0 \leq t \leq T_{*}}|u(t)|_{D^{2}}+\int_{0}^{T_{*}}\left(\left|u_{t}(t)\right|_{D_{0}^{1}}^{2}+|u(t)|_{D^{3}}^{2}\right) d s \leq c_{3},
\end{aligned}
$$

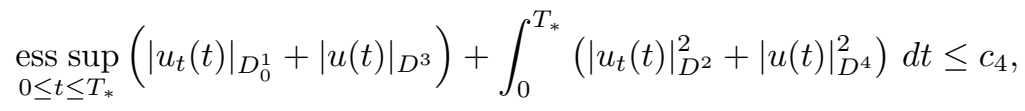

$$
\begin{aligned}
& \operatorname{ess}_{0 \leq t \leq T_{*}}\left(t^{\frac{1}{2}}\left|u_{t}(t)\right|_{D^{2}}+t^{\frac{1}{2}}|u(t)|_{D^{4}}\right)+\int_{0}^{T_{*}} t\left|u_{t t}(t)\right|_{D_{0}^{1}}^{2} d t \leq c_{5},
\end{aligned}
$$

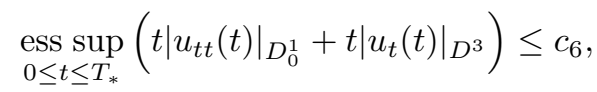

$$
\begin{aligned}
& \underset{0 \leq t \leq T_{*}}{\operatorname{ess} \sup }\left(t^{\frac{3}{2}}\left|u_{t t}(t)\right|_{D^{2}}\right)+\int_{0}^{T_{*}} t^{3}\left|u_{t t t}(t)\right|_{D_{0}^{1}}^{2} d t \leq c_{6}, \\
& \operatorname{ess}_{0 \leq t \leq T_{*}}\left(\left|\rho(t)-\rho^{\infty}\right|_{H^{3}}+\left|\rho_{t}(t)\right|_{H^{2}}+\left|\sqrt{\rho} u_{t}(t)\right|_{L^{2}}\right)+\int_{0}^{t}\left|\sqrt{\rho} u_{t t}(s)\right|_{L^{2}}^{2} d s \leq c_{6}, \\
& \operatorname{ess}_{0 \leq t \leq T_{*}}\left(t^{\frac{1}{2}}\left|\sqrt{\rho} u_{t t}\right|_{L^{2}}+t^{\frac{3}{2}}\left|\sqrt{\rho} u_{t t t}(t)\right|_{L^{2}}\right)+\int_{0}^{t} s^{2}\left|\sqrt{\rho} u_{t t t}(s)\right|_{L^{2}}^{2} d s \leq c_{6} .
\end{aligned}
$$

By virtue of these a priori estimates, we can prove the existence and regularity of a unique local classical solution $(\rho, u)$ to the original nonlinear problem following exactly the same arguments as in the proof of Theorem 1.1. We omit the details. This completes the proof of Theorem 1.3.

\section{Proof of Theorem 1.4}

To prove Theorem 1.4, we consider the following initial boundary value problem

$$
\begin{array}{cc}
\rho_{t}+\operatorname{div}(\rho u)=0 & \text { in } \quad(0, T) \times \Omega, \\
p_{t}+u \cdot \nabla p+\gamma p \operatorname{div} u=0 & \text { in } \quad(0, T) \times \Omega, \\
(\rho u)_{t}+\operatorname{div}(\rho u \otimes u)+L u+\nabla p=\rho f & \text { in } \quad(0, T) \times \Omega, \\
\left.(\rho, p, u)\right|_{t=0}=\left(\rho_{0}, p_{0}, u_{0}\right) \quad \text { in } \quad \Omega, \quad u=0 & \text { on } \quad(0, T) \times \partial \Omega, \\
(\rho, p, u)(t, x) \rightarrow\left(\rho^{\infty}, p^{\infty}, 0\right) \quad \text { as } \quad|x| \rightarrow \infty, & (t, x) \in(0, T) \times \Omega,
\end{array}
$$

where the known data $\rho_{0}, p_{0}, u_{0}$ and $f$ satisfy

$$
\begin{gathered}
\left(\rho_{0}-\rho^{\infty}, p_{0}-p^{\infty}\right) \in H^{3}, \quad \rho^{\infty} \in \overline{\mathbf{R}}_{+}, \quad p^{\infty} \in \mathbf{R}, \quad \rho_{0} \geq 0 \quad \text { in } \quad \Omega, \\
u_{0} \in D_{0}^{1} \cap D^{3}, \quad f \in L^{2}\left(0, T ; H^{2}\right) \quad \text { and } \quad f_{t} \in L^{2}\left(0, T ; L^{2}\right)
\end{gathered}
$$

and

$$
L u_{0}+\nabla p_{0}=\rho_{0}\left(f(0)+g_{2}\right) \text { for some } g_{2} \in D_{0}^{1} \text { with } \sqrt{\rho}_{0} g_{2} \in L^{2} .
$$

Theorem 1.4 is an immediate corollary of the following result. 
Theorem 6.1. Assume that the data $\rho_{0}, p_{0}, u_{0}, f$ satisfy (6.6) and (6.7). Then there exist a small time $T_{*} \in(0, T)$ and a unique strong solution $(\rho, p, u)$ to the IBVP(6.1)-(6.5) such that

$$
\begin{gathered}
\left(\rho-\rho^{\infty}, p-p^{\infty}\right) \in C\left(\left[0, T_{*}\right] ; H^{3}\right), \quad u \in C\left(\left[0, T_{*}\right] ; D_{0}^{1} \cap D^{3}\right) \cap L^{2}\left(0, T_{*} ; D^{4}\right), \\
u_{t} \in L^{\infty}\left(0, T_{*} ; D_{0}^{1}\right) \cap L^{2}\left(0, T_{*} ; D^{2}\right) \quad \text { and } \quad \sqrt{\rho} u_{t} \in L^{\infty}\left(0, T_{*} ; L^{2}\right) .
\end{gathered}
$$

Moreover, if the external force $f$ satisfies the additional regularity (1.13), then the velocity $u$ satisfies (1.14) with $T_{*}$ replaced by some $T_{* *} \in\left(0, T_{*}\right]$ and so $(\rho, p, u)$ is a classical solution of $(6.1)-(6.3)$ in $\left(0, T_{* *}\right) \times \Omega$.

Proof of Theorem 1.4 from Theorem 6.1. Let $\left(\rho_{0}, u_{0}, f\right)$ be a given data satisfying the hypotheses of Theorem 1.4. Then Theorem 6.1 guarantees the existence of a unique solution $(\rho, p, u)$ to the $\operatorname{IBVP}(6.1)-(6.5)$ with the initial data $\left(\rho_{0}, p_{0}, u_{0}\right)$, where $p_{0}=A \rho_{0}^{\gamma}$ and $p^{\infty}=A\left(\rho^{\infty}\right)^{\gamma}$.

To prove Theorem 1.4, we have only to show that $p=A \rho^{\gamma}$. Let us denote $\bar{p}=p-A \rho^{\gamma}$. Then using (6.1), (6.2) and (6.4) together with the fact that $\gamma>1$, we deduce that

$$
\left\{\begin{array}{c}
\bar{p}_{t}+u \cdot \nabla \bar{p}+\gamma \bar{p} \operatorname{div} u=0 \quad \text { in } \quad\left(0, T_{*}\right) \times \Omega, \\
\bar{p}(0)=0 \quad \text { in } \quad \Omega, \quad \bar{p} \in C\left(\left[0, T_{*}\right] ; H^{1}\right) .
\end{array}\right.
$$

Hence by virtue of a standard energy method, we easily conclude that $\bar{p}=0$ in $\left(0, T_{*}\right) \times \Omega$. This completes the proof of Theorem 1.4.

Finally, we turn to the proof of Theorem 6.1. For this purpose, we follow the same strategy as in the previous sections. Let us consider the following uncoupled linearized problem

$$
\begin{array}{cr}
\rho_{t}+\operatorname{div}(\rho v)=0 & \text { in } \quad(0, T) \times \Omega, \\
p_{t}+v \cdot \nabla p+\gamma p \operatorname{div} v=0 & \text { in } \quad(0, T) \times \Omega, \\
\rho u_{t}+L u+\nabla p=\rho(f-v \cdot \nabla v) & \text { in } \quad(0, T) \times \Omega, \\
\left.(\rho, p, u)\right|_{t=0}=\left(\rho_{0}, p_{0}, u_{0}\right) \quad \text { in } \quad \Omega, \quad u=0 & \text { on } \quad(0, T) \times \partial \Omega, \\
(\rho, p, u)(t, x) \rightarrow\left(\rho^{\infty}, p^{\infty}, 0\right) \quad \text { as } \quad|x| \rightarrow \infty, & (t, x) \in(0, T) \times \Omega,
\end{array}
$$

where $v$ is a known vector field such that

$$
v \in C\left([0, T] ; D_{0}^{1} \cap D^{3}\right) \cap L^{2}\left(0, T ; D^{4}\right), \quad v_{t} \in L^{\infty}\left(0, T ; D_{0}^{1}\right) \cap L^{2}\left(0, T ; D^{2}\right) .
$$

Note that the proof of Lemma 2.1 can be used without any essential change to deduce the corresponding result for the linear hyperbolic problem (6.9), (6.11) and (6.12). Hence adapting the proof of Lemma 3.1, we can prove

Lemma 6.2. Let $\Omega$ be a bounded domain in $\mathbf{R}^{3}$ with smooth boundary. In addition to (6.6), (6.7) and (6.13), we assume that $\rho_{0} \geq \delta$ in $\Omega$ for some constant $\delta>0$. Then there exists a unique solution $(\rho, p, u)$ to the linearized problem (6.8)-(6.11) 
such that

$$
\begin{gathered}
\rho, p \in C\left([0, T] ; H^{3}\right), \quad \rho_{t}, p_{t} \in C\left([0, T] ; H^{2}\right), \\
u \in C\left([0, T] ; H_{0}^{1} \cap H^{3}\right) \cap L^{2}\left(0, T ; H^{4}\right), \\
u_{t} \in C\left([0, T] ; H_{0}^{1}\right) \cap L^{2}\left(0, T ; H^{2}\right), \\
u_{t t} \in L^{2}\left(0, T ; L^{2}\right) \quad \text { and } \quad \rho \geq \underline{\delta} \quad \text { on } \quad[0, T] \times \bar{\Omega} .
\end{gathered}
$$

for some constant $\underline{\delta}>0$.

Moreover, from Lemma 2.1 and its proof, it follows that

$$
\begin{aligned}
& \left|\rho(t)-\rho^{\infty}\right|_{H^{3}} \leq\left(\left|\rho_{0}-\rho^{\infty}\right|_{H^{3}}+\rho^{\infty}\right) \exp \left(C \int_{0}^{t}|v(s)|_{D_{0}^{1} \cap D^{4}} d s\right), \\
& \left|p(t)-p^{\infty}\right|_{H^{3}} \leq\left(\left|p_{0}-p^{\infty}\right|_{H^{3}}+\left|p^{\infty}\right|\right) \exp \left(C \int_{0}^{t}|v(s)|_{D_{0}^{1} \cap D^{4}} d s\right)
\end{aligned}
$$

and

$$
\inf _{\Omega} \rho(t) \geq\left(\inf _{\Omega} \rho_{0}\right) \exp \left(-C \int_{0}^{t}|v(s)|_{D_{0}^{1} \cap D^{4}} d s\right)
$$

for $0 \leq t \leq T$. Here we denote by $C$ a generic positive constants depending only on the fixed constants $\mu, \lambda, T, \gamma$ and the norm of $f$.

Hence adapting the proof of Lemma 4.1, we can also prove the key lemma.

Lemma 6.3. Let us choose a constant $c_{0}>1$ so that

$$
1+\rho^{\infty}+\left|p^{\infty}\right|+\left|\left(\rho_{0}-\rho^{\infty}, p_{0}-p^{\infty}\right)\right|_{H^{3}}+\left|u_{0}\right|_{D_{0}^{1}}+\left|\sqrt{\rho}_{0} g_{2}\right|_{L^{2}}+\left|g_{2}\right|_{D_{0}^{1}}<c_{0} .
$$

Then there exist positive constants $T_{*} \in(0, T)$ and $c_{i}$ 's, depending only on $c_{0}$ and the parameters of $C$, with the following property:

If $v$ is a vector field satisfying the regularity (6.13) with $T$ replaced by $T_{*}$ and the estimate

$$
\begin{array}{r}
|v(0)|_{D_{0}^{1} \cap D^{3}} \leq c_{1}, \\
\sup _{0 \leq t \leq T_{*}}|v(t)|_{D_{0}^{1}}+\int_{0}^{T_{*}}|v(t)|_{D^{2}}^{2} d t \leq c_{2}, \\
\sup _{0 \leq t \leq T_{*}}|v(t)|_{D^{2}}+\int_{0}^{T_{*}}\left(\left|v_{t}(t)\right|_{D_{0}^{1}}^{2}+|v(t)|_{D^{3}}^{2}\right) d t \leq c_{3}, \\
\operatorname{ess~sup}_{0 \leq t \leq T_{*}}\left(\left|v_{t}(t)\right|_{D_{0}^{1}}+|v(t)|_{D^{3}}\right)+\int_{0}^{T_{*}}\left(\left|v_{t}(t)\right|_{D^{2}}^{2}+|v(t)|_{D^{4}}^{2}\right) d t \leq c_{4},
\end{array}
$$

then there exists a unique solution $(\rho, p, u)$ to the linearized problem (6.8)-(6.12) satisfying the regularity

$$
\begin{gathered}
\left(\rho-\rho^{\infty}, p-p^{\infty}\right) \in C\left(\left[0, T_{*}\right] ; H^{3}\right), \quad u \in C\left(\left[0, T_{*}\right] ; D_{0}^{1} \cap D^{3}\right) \cap L^{2}\left(0, T_{*} ; D^{4}\right), \\
u_{t} \in L^{\infty}\left(0, T_{*} ; D_{0}^{1}\right) \cap L^{2}\left(0, T_{*} ; D^{2}\right) \quad \text { and } \quad \sqrt{\rho} u_{t} \in L^{\infty}\left(0, T_{*} ; L^{2}\right)
\end{gathered}
$$


and the estimate

$$
\begin{aligned}
& \sup _{0 \leq t \leq T_{*}}|u(t)|_{D_{0}^{1}}+\int_{0}^{T_{*}}|u(t)|_{D^{2}}^{2} d t \leq c_{2} \\
& \sup _{0 \leq t \leq T_{*}}|u(t)|_{D^{2}}+\int_{0}^{T_{*}}\left(\left|u_{t}(t)\right|_{D_{0}^{1}}^{2}+|u(t)|_{D^{3}}^{2}\right) d t \leq c_{3}, \\
& \operatorname{ess}_{0 \leq t \leq T_{*}}\left(\left|u_{t}(t)\right|_{D_{0}^{1}}+|u(t)|_{D^{3}}\right)+\int_{0}^{T_{*}}\left(\left|u_{t}(t)\right|_{D^{2}}^{2}+|u(t)|_{D^{4}}^{2}\right) d t \leq c_{4},
\end{aligned}
$$

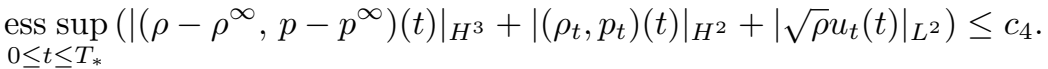

The first part of Theorem 6.1 can be deduced from this key lemma following the same arguments as in the proof of Theorem 1.1. Combining this idea and the proof of Theorem 1.3, we can also prove the remaining part of the theorem. We omit its details.

\section{REFERENCES}

[1] J.L. Boldrini, M. A. Rojas-Medar and E. Fernández-Cara, Semi-Galerkin approximation and strong solutions to the equations of the nonhomogeneous asymmetric fluids, J. Math. Pures Appl. 82 (2003), 1499-1525.

[2] Y. Cho and H. Kim, Existence results for viscous polytropic fluids with vacuum, to appear in J. Differential Equations, Hokkaido University Preprint Series in Mathematics No. 675.

[3] Y. Cho, H. J. Choe and H. Kim, Unique solvability of the initial boundary value problems for compressible viscous fluids, J. Math. Pures Appl. 83 (2004), 243-275.

[4] H. J. Choe and H. Kim,Strong solutions of the Navier-Stokes equations for isentropic compressible fluids, J. Differential Equations 190 (2003), 504-523.

[5] H. J. Choe and H. Kim, Global existence of the radially symmetric solutions of the NavierStokes equations for the isentropic compressible fluids, Math. Methods Appl. Sci. 28 (2005), 1-28.

[6] R. Danchin, Global existence in critical spaces for compressible Navier-Stokes equations, Invent. math. 141(2000), 579-614.

[7] B. Desjardins, Regularity of weak solutions of the compressible isentropic Navier-Stokes equations, Comm. Partial Differential Equations 22 (1997), 977-1008.

[8] E. Feireisl, Compressible Navier-Stokes equations with a non-monotone pressure law, J. Differential Equations 184 (2002), 97-108.

[9] E. Feireisl, A. Novotný and H. Petzeltová, On the existence of globally defined weak solutions to the Navier-Stokes equations, J. Math. fluid Mech. 3 (2001), 358-392.

[10] E. Feireisl, A. Novotný and H. Petzeltová, On the domain dependence of solutions to the compressible Navier-Stokes equations of a barotropic fluid, Math. Methods Appl. Sci. 25 (2002), 1045-1073.

[11] G.P. Galdi, "An introduction to the mathematical theory of the Navier-Stokes equations," Springer-Verlag, New York, 1994.

[12] D. Hoff, Global solutions of the Navier-Stokes equations for multidimensional compressible flow with discontinuous initial data, J. Differential Equations 120(1995), 215-254.

[13] N. Itaya, On the Cauchy problem for the system of fundamental quations describing the movement of compressible viscous fluids, Kodai Math. Sem. Rep. 23(1971), 60-120.

[14] S. Jiang and P. Zhang, On spherically symmetric solutions of the compressible isentropic Navier-Stokes equations, Comm. Math. Phys. 215 (2001), 559-581.

[15] S. Jiang and P. Zhang, Axisymmetric solutions of the $3 D$ Navier-Stokes equations for compressible isentropic fluids, J. Math. Pures Appl. 82(2003), 949-973.

[16] P.L. Lions, Existence globale de solutions pour les équations de Navier-Stokes compressibles isentropiques, C.R. Acad. Sci. 316 (1993), 1335-1340.

[17] P.L. Lions, "Mathematical Topics in Fluid Mechanics Vol. 2," Clarendon Press, Oxford, 1998. 
[18] A. Matsumura and T. Nishida, The initial value problem for the equations of motion of viscous and heat-conductive gases, J. Math. Kyoto Univ. 20 (1980), 67-104.

[19] A. Matsumura and T. Nishida, The initial boundary value problems for the equations of motion of compressible and heat-conductive fluids, Comm. Math. Phys. 89 (1983), 445-464.

[20] J. Nash, Le probléme de Cauchy pour les équations différentielles d'un fluide général, Bull. Soc. Math. France 90(1962), 487-497.

[21] R. Salvi and I. Straškraba, Global existece for viscous compressible fluids and their behavior as $t \rightarrow \infty$, J. Fac. Sci. Univ. Tokyo Sect. IA, Math. 40 (1993), 17-51.

[22] J. Simon, Compact sets in the space $L^{p}(0, T ; B)$, Ann. Mat. Pura Appl. 146 (1987), 65-96.

23] V. A. Solonnikov, Solvability of the initial boundary value problem for the equation of a viscous compressible fluid, J. Sov. Math. 14 (1980), 1120-1133.

[24] A. Tani, On the first initial-boundary value problem of compressible viscous fluid motion, Publ. Res. Inst. Math. Sci. Kyoto Univ. 13 (1971), 193-253.

[25] R. Temam, Behaviour at time $t=0$ of the solutions of semi-linear evolution equations, J. Differential Equations 43 (1982), 73-92.

26] R. Temam, "Navier-Stokes equations: Theory and Numerical analysis," North-Holland, Amsterdam, 1984.

[27] A. Valli, An existence theorem for compressible viscous fluids, Ann. Mat. Pura Appl. (IV) 130 (1982), 197-213 ; (IV) 132 (1982), 399-400.

[28] A. Valli, Periodic and stationary solutions for compressible Navier-Stokes equations via a statiblity method, Ann. Sc. Norm. Super. Pisa, Cl. Sci. 10 (1983), 607-647.

[29] A. Valli and W. M. Zajaczkowski, Navier-Stokes equations for compressible fluids: global existence and qualitative properties of the solutions in the general case, Commun. Math. Phys. 103 (1986), 259-296.

[30] Z. Xin, Blowup of smooth solutions to the compressible Navier-Stokes equation with compact density, Comm. Pure Appl. Math. 51 (1998), 229-240.

Department of Mathematics, Hokkaido University, Kita 8 Nishi 5, Sapporo 060-0808, JAPAN

E-mail address: ygcho@math.sci.hokudai.ac.jp

School of mathematics, Korea Institute for Advanced Study, Seoul, 130-722, RepubLIC OF KOREA

E-mail address: khs319@kias.re.kr 